\title{
Oscillator Strengths for Ultraviolet Lines of Fe I
}

\author{
Charles H. Corliss and Brian Warner*
}

(April 1, 1966)

\begin{abstract}
Relative intensities and oscillator strengths for 2000 ultraviolet lines of $\mathrm{Fe}$ I from several investigations between 2080 and $4150 \AA$ have been critically discussed and adjusted to a uniform absolute scale. New values for many lines not measured heretofore are reported.
\end{abstract}

Key words: Atomic spectra, iron, oscillator strengths, transition probability, ultraviolet lines of Fe I.

\section{Introduction}

During the past 30 years, numerous investigations of intensities and oscillator strengths in the ultraviolet part of the iron spectrum have been carried out. The great variety of forms in which they are reported makes comparisons difficult and obscures systematic errors which could otherwise be discovered and perhaps corrected. Furthermore, the fragmentary nature of these reports often leaves serious gaps in our knowledge of the line strength characteristics of the spectrum. Collection of these published reports into a single compilation not only permits the discovery of errors but discloses the need for certain additional work to be done. In 1964 Corliss and Warner made such a compilation for Fe I in the region 3100 to $9000 \AA$ and supplied many new measurements in the region 4000 to $9900 \AA$. This provides nearly complete information for $\mathrm{Fe} I$ in the regions of interest to astrophysicists who obtain their data through the atmosphere but there remain many lacunae in the ultraviolet part of the spectrum that is of special interest to laboratory spectroscopists and space astrophysicists.

It is the purpose of this paper to extend that work as far as possible into the ultraviolet. To that end we have assembled such published ultraviolet data as were overlooked in our previous compilation or have since appeared, adjusted them to the same absolute scale and supplemented them with new values for many lines not previously measured.

There are in general three different methods which have been used to measure intensities and oscillator strengths in spectra such as Fe I. The three methods find their optimum utility in the measurement of lines of different intensity ranges and of different degrees of excitation. The hook method, which is supposed to provide the most accurate measurements, is limited to the strong lines of the spectrum. Because of

*University of London Observatory, London, N.W. 7, England. the nature of the measurement to be made, the method of total absorption is less precise and consequently may be less accurate than the hook method, but it does permit the measurement of fainter lines. However, both of these methods, because they depend on furnace excitation do not produce lines whose lower levels are far above the ground state of the atom. To observe these lines emission methods are generally used. In emission methods, precise measurements of intensities can usually be made but the population distribution amongst the energy levels of the atoms cannot always be accurately specified. This is in contrast to the first two methods in which thermal equilibrium is assured by furnace excitation.

Work on the ultraviolet spectrum of $\mathrm{Fe}_{\mathrm{I}}$ which has already been published includes all of the stronger lines. These have been measured by all three of the methods mentioned above. To supplement this work and to complete the description of the intensity structure of $\mathrm{Fe}$ I the faint lines in the ultraviolet must be measured. We have done this by means of observations in emission, using arcs and sparks which reveal the faintest lines.

\section{Published Data}

The previously published data on the ultraviolet part of the first spectrum of iron, Fe I, discussed in this section, is summarized in table 1. A number of measurements made prior to 1962, those of King and King [1938]; Carter [1949]; Mitrofanova [1952]; Aarts, Harting, and Bakker [1954]; Allen and Asaad [1957]; and Hefferlin [1959], have been discussed by Corliss and Warner [1964] and are not further discussed here. The early work of van Milaan [1926] reports measurements of 69 lines in a $1.5 \mathrm{~A}$ iron arc and 61 of them in a spark. Comparison with other measurements shows that the lines of the stronger half of the group have been weakened by self-absorption, consequently we have not used van Milaan's data. 
TABLE 1. Published data on intensities and oscillator strengths in the ultraviolet part of Fe I discussed in this paper

\begin{tabular}{|c|c|c|c|c|c|c|c|c|}
\hline Reference & Date & $\begin{array}{l}\text { Wavelength } \\
\text { range }\end{array}$ & $\begin{array}{l}\text { No. of } \\
\text { lines }\end{array}$ & $\begin{array}{l}\text { Number } \\
\text { included }\end{array}$ & Method & $\begin{array}{l}\text { Quantity } \\
\text { reported }\end{array}$ & Temperature & $\begin{array}{c}\text { Ref. } \\
\text { symbol }\end{array}$ \\
\hline van Milaan & 1926 & $\begin{array}{c}\stackrel{\AA}{A} \\
3680-3800\end{array}$ & 69 & & arc & intensity & ${ }^{\circ} \mathrm{K}$ & \\
\hline Sobolev & 1943 & $2585-3648$ & 15 & 15 & arc & & 5300 & S3 \\
\hline Sobolev & 1943 & $2912-3723$ & 32 & 32 & $\operatorname{arc}$ & $g f$ & & $\mathrm{~S} 5$ \\
\hline Gottschalk & 1948 & $3265-4635$ & 122 & & calc. & & ......... & $\mathrm{G}$ \\
\hline Crosswhite & 1958 & $3200-4148$ & 1060 & 1000 & $\begin{array}{l}\text { hollow } \\
\text { cathode }\end{array}$ & intensity & & $\mathrm{HC}$ \\
\hline Corliss and Bozman & 1962 & $2084-3100$ & 220 & 215 & arc & $\log g f$ & 5100 & CB \\
\hline Morozova, Startsev, and Frish & 1962 & $2987-4144$ & 117 & 117 & arc & $\log g f$ & 4500 & MSF \\
\hline Margoshes and Scribner & 1963 & $2912-4144$ & 105 & 105 & $\operatorname{arc}$ & $g f$ & 5510 & MS \\
\hline Corliss and $\mathrm{W}$ arner & 1964 & $3100-4148$ & 685 & 680 & & $\log g f$ & & $\mathrm{CW}$ \\
\hline Penkin & 1964 & $2937-4427$ & 56 & 55 & hoo & $f$ & $2000-2500$ & PP \\
\hline Valters, Nikonova, and Startsev & 1964 & $2778-3930$ & 57 & 57 & absorpt. & $\log g f$ & $1750-2020$ & VNS \\
\hline Valters and Startsev & 1964 & $2719-3930$ & 89 & 89 & hook & $\log g f$ & 2200 & VS \\
\hline King, Olsen, and Corliss & 1965 & $2502-3201$ & 300 & 290 & absorpt. & $\log g f$ & $1600-3000$ & KOC \\
\hline
\end{tabular}

\subsection{Calculated Values}

Two papers have been published dealing with extensive theoretical calculations of line strengths in Fe I. The first was by Gottschalk [1948] who computed line strengths in intermediate coupling for the $3 d^{7}\left({ }^{4} \mathrm{P}\right) 4 s-3 d^{7}\left({ }^{4} \mathrm{P}\right) 4 p$ and $3 d^{7}\left({ }^{4} \mathbf{F}\right) 4 s-3 d^{7}\left({ }^{4} \mathbf{F}\right) 4 p$ transitions. To test these values we have plotted $\log S / \lambda$ from Gottschalk versus log $g$ from Corliss and Bozman [1962] in figure 1. The figure shows that calculations for the ${ }^{4} \mathrm{P}$ parent are unreliable but that, except for

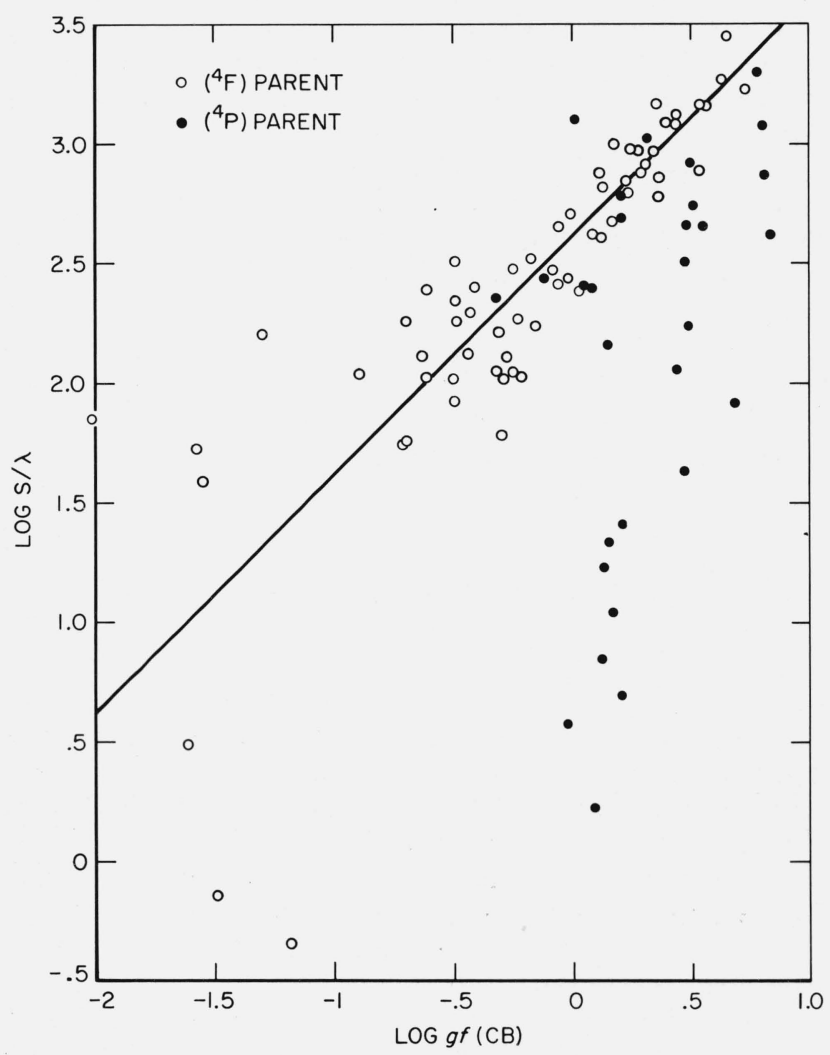

Figure 1. Comparison of log gf-values from Gottschalk [1948] with those from Corliss and Bozman [1962]. lines for which $\log g f<-1.0$, the calculations for the ${ }^{4} \mathrm{~F}$ parent are in agreement with the measured values. According to Gottschalk, the disagreement in the case of the ${ }^{4} \mathrm{P}$ parent is caused by the fact that the $L$ and the $S$ of the ${ }^{4} \mathrm{P}$ core are not good quantum numbers. The lack of agreement for faint lines (in the case of the ${ }^{4} \mathrm{~F}$ parent) seems to be typical of calculated values. Since only 57 of his lines are of use to us and since these have all been measured many times by others, we have omitted them from our tabulation. It should be noted, however, by those interested in theoretical calculation of line strengths that the method used by Gottschalk has led in certain cases to remarkably accurate values.

Recently Shore [1965] has investigated simple $L S$ coupling line and multiplet strengths in Fe I and compared them with observed values. He found that the standard deviation within a multiplet was 30 percent or less (i.e., within the experimental error) for about half the multiplets. The fluctuations among multiplets in transition arrays were considerably larger, sometimes amounting to several orders of magnitude. Many of the observed Fe I lines are intersystem transitions which are not predicted in this scheme.

In view of the evident uncertainties in calculated line strengths for $\mathrm{Fe} \mathrm{I}$, it seems that at present we are forced to rely almost entirely on measured values. Further investigation by theoreticians of calculations by the method of intermediate coupling would appear to be very much worthwhile, however.

\subsection{Russian Measurements}

Over the past 50 years, since the introduction of the hook method into the physical laboratories at the University of Leningrad by Rozhdestvenskii, Russian physicists have conducted an outstanding sustained effort in the measurement of oscillator strengths. A number of their papers have been concerned with Fe I. In 1964 Prokofiev, Nikonova, Gruzdev, and Frish published a review of the subject entitled "Oscillator Strengths in the Spectrum Fe I." In 
TABLE 2. Russian measurements of $\log$ gf for ultraviolet lines of $\mathrm{Fe} \mathrm{I}$

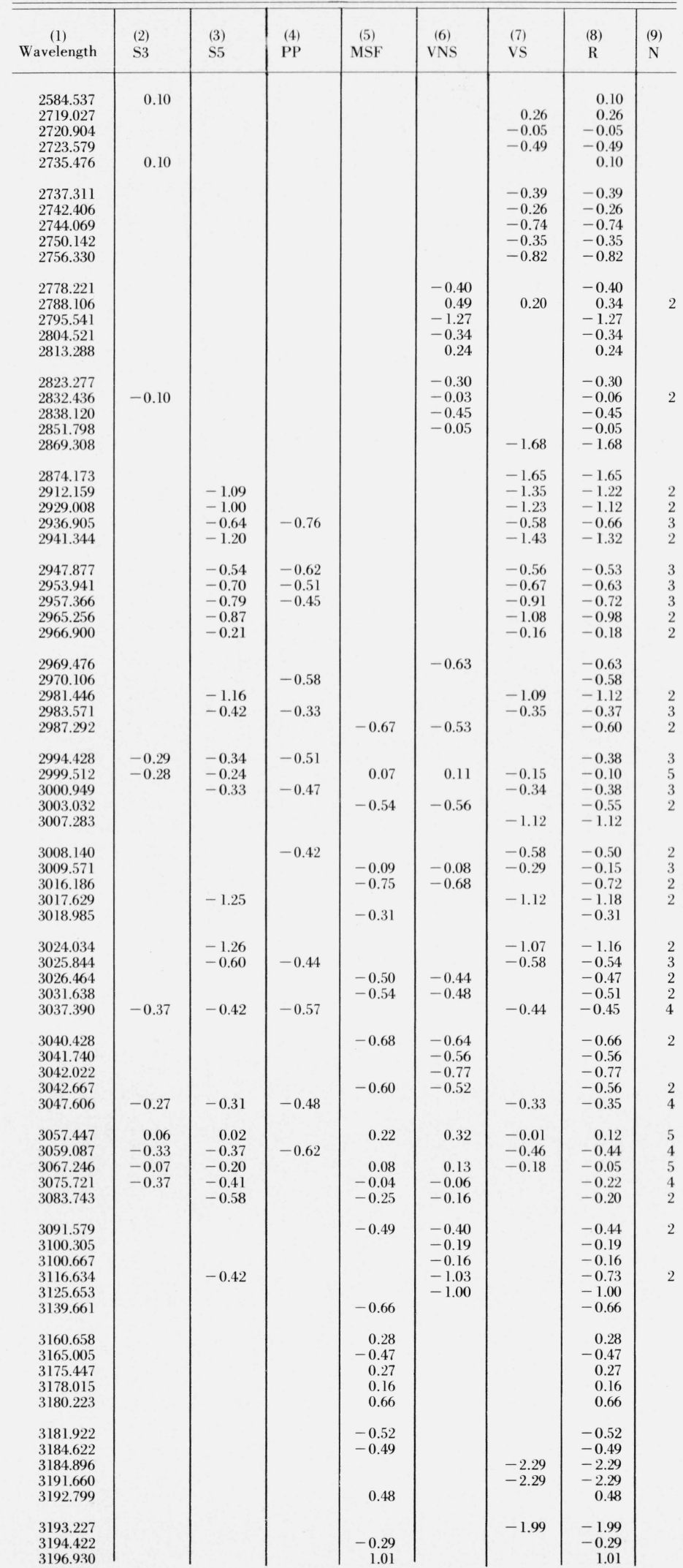


TABLE 2. Russian measurements of log gf for ultraviolet lines of Fe I-Continued

\begin{tabular}{|c|c|c|c|c|c|c|c|c|}
\hline $\begin{array}{c}\text { (1) } \\
\text { Wavelength }\end{array}$ & $\begin{array}{l}(2) \\
\text { S3 }\end{array}$ & $\begin{array}{l}(3) \\
\text { S5 }\end{array}$ & $\begin{array}{l}(4) \\
\text { PP }\end{array}$ & $\stackrel{(5)}{\mathrm{MSF}}$ & $\begin{array}{c}(6) \\
\text { VNS }\end{array}$ & $\begin{array}{l}\text { (7) } \\
\text { VS }\end{array}$ & $\begin{array}{l}(8) \\
\mathrm{R}\end{array}$ & $\begin{array}{l}(9) \\
\mathrm{N}\end{array}$ \\
\hline $\begin{array}{l}3199.530 \\
3200.475\end{array}$ & & & & $\begin{array}{l}0.57 \\
0.70\end{array}$ & & & $\begin{array}{l}0.57 \\
0.70\end{array}$ & \\
\hline 3205.400 & & & & 0.62 & & & 0.62 & \\
\hline 3210.830 & & & & 0.53 & & & 0.53 & \\
\hline 3214.044 & & & & 1.05 & & & 1.05 & \\
\hline 3215.940 & & & & 0.64 & & & 0.64 & \\
\hline 3219.581 & & & & 0.74 & & & 0.74 & \\
\hline 3221.931 & & & & -0.98 & & & -0.98 & \\
\hline 3222.069 & & & & 1.09 & & & 1.09 & \\
\hline 3225.789 & & & & 1.26 & & & 1.26 & \\
\hline 3227.063 & & & & -0.38 & & & -0.38 & \\
\hline 3244.190 & & & & 0.68 & & & 0.68 & \\
\hline 3265.618 & & & & 0.42 & & & 0.42 & \\
\hline 3271.001 & & & & 0.46 & & & 0.46 & \\
\hline 3284.589 & & & - & -0.27 & & & -0.27 & \\
\hline 3286.754 & & & & $\begin{array}{r}0.90 \\
0.23\end{array}$ & & & $\begin{array}{r}0.90 \\
0.23\end{array}$ & \\
\hline 3292.591 & & & & 0.23 & & & 0.23 & \\
\hline 3305.972 & & & & 0.82 & & & 0.82 & \\
\hline 3306.356 & & & & 0.87 & & & 0.87 & \\
\hline 3379.021 & & & & -0.20 & & & -0.20 & \\
\hline 3383.692 & & & & -0.21 & & & -0.21 & \\
\hline 3392.654 & & & & 0.54 & & & 0.54 & \\
\hline 3394.585 & & & & -0.21 & & & -0.21 & \\
\hline 3399.336 & & & & 0.61 & & & 0.61 & \\
\hline 3406.802 & & & & 0.21 & & & 0.21 & \\
\hline 3413.134 & & & & 0.82 & & & 0.82 & \\
\hline 3417.843 & & & & 0.48 & & & 0.48 & \\
\hline 3418.507 & & & & 0.40 & & & 0.40 & \\
\hline 3422.658 & & & & 0.35 & & & 0.35 & \\
\hline 3424.286 & & & & 0.43 & & & 0.43 & \\
\hline 3426.383 & & & & 0.05 & & & 0.05 & \\
\hline 3426.637 & & & & 0.05 & & & 0.05 & \\
\hline 3427.121 & & & & 0.98 & & & 0.98 & \\
\hline 3428.195 & & & & 0.34 & & & 0.34 & \\
\hline 3440.607 & & & & & & $\begin{array}{l}-0.49 \\
-0.79\end{array}$ & $\begin{array}{l}-0.49 \\
-0.79\end{array}$ & \\
\hline 3440.990 & & & & & & -0.79 & -0.79 & \\
\hline 3443.878 & & & & & & -1.19 & -1.19 & \\
\hline 3445.151 & & & & 0.63 & & & 0.63 & \\
\hline 3447.280 & & & & 0.04 & & & 0.04 & \\
\hline 3450.330 & & & & 0.14 & & & 0.14 & \\
\hline 3451.917 & & & & 0.14 & & & 0.14 & \\
\hline 3465.862 & & & & & & -1.00 & -1.00 & \\
\hline 3471.267 & & & & -0.21 & & & -0.21 & \\
\hline 3475.451 & & & & & & -0.89 & -0.89 & \\
\hline 3476.704 & & & & & -1.14 & -1.29 & -1.22 & 2 \\
\hline 3477.856 & & & & -0.78 & & -0.93 & $\begin{array}{l}-0.78 \\
-0.93\end{array}$ & \\
\hline 3490.575 & & & & & & -0.93 & -0.93 & \\
\hline 3497.842 & & -1.00 & -1.52 & & -1.38 & -1.36 & -1.32 & 4 \\
\hline 3521.263 & & & -0.46 & & & & -0.46 & \\
\hline 3526.042 & & & & & -1.59 & & -1.59 & \\
\hline 3545.639 & & & & $\begin{array}{r}0.59 \\
-0.16\end{array}$ & & & $\begin{array}{r}0.59 \\
-0.16\end{array}$ & \\
\hline 3547.203 & & & & -0.16 & & & -0.16 & \\
\hline 3552.828 & & & & 0.36 & & & 0.36 & \\
\hline 3558.517 & -0.35 & -0.59 & -0.22 & & & & -0.39 & 3 \\
\hline 3565.381 & & -0.16 & 0.02 & & & 0.12 & -0.01 & 3 \\
\hline 3565.583 & & & & 0.73 & & & 0.73 & \\
\hline 3568.423 & & & & -0.13 & & & -0.13 & \\
\hline 3570.100 & & 0.19 & 0.34 & & 0.48 & 0.40 & 0.35 & 4 \\
\hline 3571.995 & & & & 0.87 & & & 0.87 & \\
\hline 3575.118 & & & & 0.03 & & & 0.03 & \\
\hline 3575.249 & & & & 0.18 & & & 0.18 & \\
\hline 3575.976 & & & & 0.10 & & & 0.10 & \\
\hline 3578.380 & & & & 0.96 & & & 0.96 & \\
\hline 3581.195 & & & 0.51 & & 0.69 & 0.63 & 0.61 & 3 \\
\hline 3588.918 & & & & 0.19 & & & 0.19 & \\
\hline 3594.632 & & & & 0.79 & & & 0.79 & \\
\hline 3595.308 & & & & -0.04 & & & -0.04 & \\
\hline 3602.08 & & & & 0.00 & & & 0.00 & \\
\hline 3608.861 & 0.24 & & 0.19 & & & 0.21 & 0.21 & 3 \\
\hline 3610.159 & & & & 1.18 & & & 1.18 & \\
\hline 3618.769 & 0.24 & & 0.27 & & & 0.28 & 0.26 & 3 \\
\hline 3631.103 & & & & 0.10 & & & 0.10 & \\
\hline 3631.465 & & & 0.17 & & & 0.27 & 0.22 & 2 \\
\hline 3637.862 & & & & 0.09 & & & 0.09 & \\
\hline 3643.627 & & & & 0.33 & & & 0.33 & \\
\hline $\begin{array}{l}3647.844 \\
3649.304\end{array}$ & 0.05 & & 0.08 & & & $\begin{array}{r}0.12 \\
-2.75\end{array}$ & $\begin{array}{r}0.08 \\
-2.75\end{array}$ & 3 \\
\hline
\end{tabular}


TABLE 2. Russian measurements of log gf for ultraviolet lines of Fe I-Continued

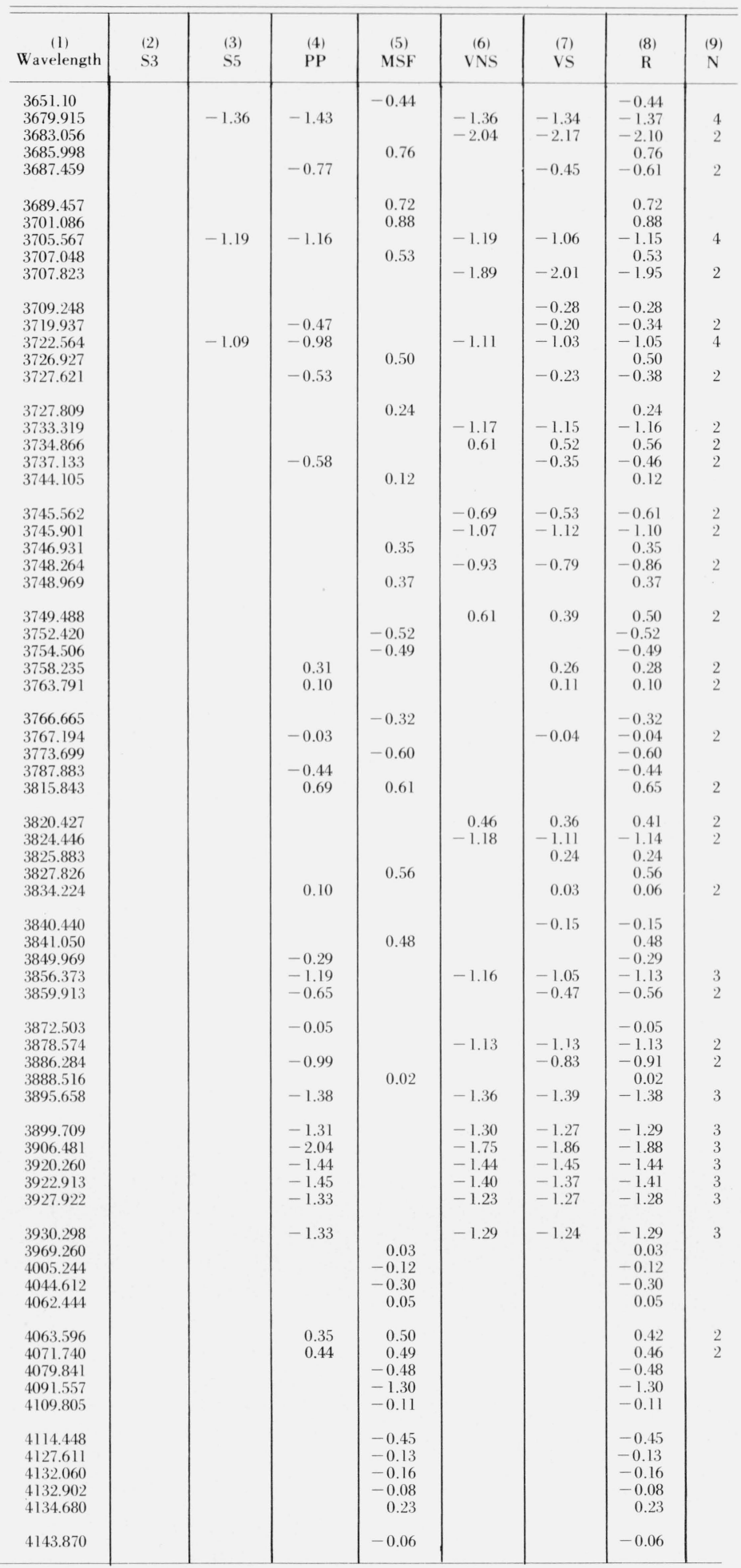


it they review critically 18 papers on the subject and tabulate mean values of $\log g f$ for 1167 lines of $\mathrm{Fe} \mathrm{I}$ reported in those papers.

There are five Russian papers that report original observations in the ultraviolet region of $\mathrm{Fe} \mathrm{I}$; they are discussed individually below. Because none of them report very extensive measurements we have tabulated them separately in table 2 . The mean value of $\log g f$ for each line is given in column 8 of table 2 and entered into table 3 , the complete tabulation, in column 10 headed R. The number of observations in table 2 is listed in column 10 of table 3 as a superscript following the $\mathrm{R}$ value. All values are normalized to the scale of Corliss and Bozman.

\section{a. Sobolev [1943]}

Sobolev published two sets of measurements made using a $3 \mathrm{~A} \mathrm{~d}$-c arc between copper electrodes containing 0.3 percent iron and 0.3 percent tin. From intensity ratios of tin lines he determined a temperature of $5300{ }^{\circ} \mathrm{K}$. Since the two sets overlap, we have listed them in columns 2 and 3 of table 2 under the headings S3 and S5. Sobolev's values compare well with other reliable measurements. In discussing the discrepancies between his own measurements and those of King and King, he states, "It appears that the conditions under which the measurements of King were made were not conditions of thermal equilibrium for the excited levels." Since that time, numerous investigators, e.g., Crosswhite [1958]; Prokofiev, Nikonova, Gruzdev, and Frish [1964]; or Margoshes and Scribner [1963], have shown that the discrepancy is due to scattered light in the short wavelength end of the Kings' spectra, which made their values too small near $3000 \AA$. The disagreement is shown in figure 3 of Margoshes and Scribner's paper.

\section{b. Parchevsky and Penkin [1954], Penkin [1964]}

Parchevsky and Penkin [1954] published relative oscillator strengths for 56 lines of Fe I which they had measured by the hook method at the University of Leningrad. With reference to the accuracy of the hook method, the authors state that the quantity $\mathrm{Nf}$ can be determined with an error of from 3 to 20 percent, depending on the distance between the peaks of the hooks, and that the error in the quantity $N$ depends on the uncertainty in the temperature in the Boltzmann formula and in this case can be as much as 15 percent. This would imply that the maximum error was not greater than 35 percent.

In 1964 Penkin published a review and summary of his past work. In the case of iron, substantial changes were made in many of the $f$-values. Some of the values were changed by factors as large as five. The value for 3872.503 was not changed and is about 3 times as large as the six other values for that line in table 3. For conversion to the CB scale 3.70 was subtracted from the new values. The new values are compared with those of Corliss and Bozman in figure 2 and the converted values are listed in column 4 of table 2.
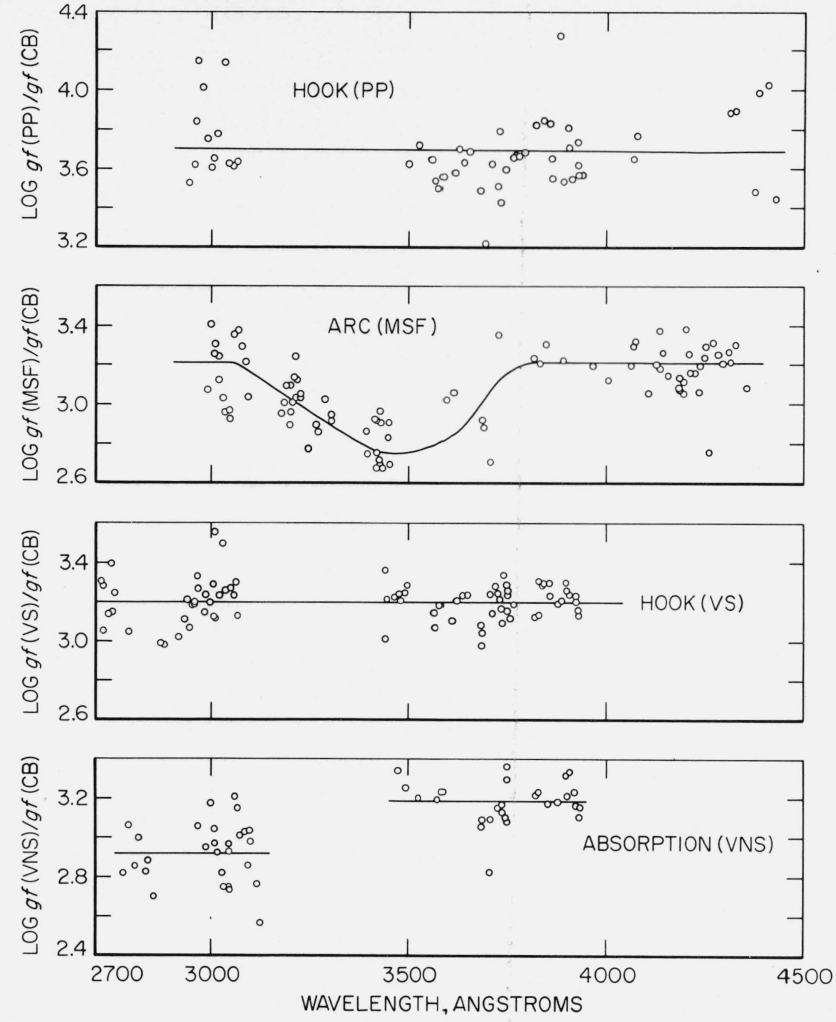

Figure 2. Comparison of some Russian measurements of $\log$ gfvalues with those from Corliss and Bozman [1962] in the wavelength range 2700 to $4500 \AA$.

\section{c. Morosova, Startsev, and Frish [1962]}

Using a free burning arc in air, Morosova, Startsev, and Frish have measured oscillator strengths for Fe I, 117 of which are for lines below $4150 \AA$. By studying the self-absorption in the arc, they found that they could measure the intensities of lines arising from the low levels that lie above the $a^{5} \mathrm{~F}$ level if they used an iron anode, a copper cathode and a current of $1.2 \mathrm{~A}$. By comparison of their intensities with those of Crosswhite [1950], they determined a temperature of $4500^{\circ} \mathrm{K}$ for their arc. They carried out their photographic photometry with a tungsten lamp, the crater of a carbon arc, and the calibrated continuum from a krypton lamp. A plot of the ratio of their values to those of Corliss and Bozman, in figure 2, shows that their values are too small in the region from 3100 to $3700 \AA$. An appropriate correction taken from figure 2 was applied and their values tabulated in table 2 under the heading MSF.

\section{d. Valters, Nikonova, and Startsev [1964]}

Measuring the equivalent widths of lines absorbed by iron vapor in a furnace, Valters, Nikonova, and Startsev determined oscillator strengths for 28 lines in the region 2750 to $3150 \AA$ and 28 lines in the region 3450 to $3950 \AA$. They took special precautions to filter out scattered light from longer wavelengths in their spectrograph. Nevertheless, a plot of the ratio 
of their values to those of Corliss and Bozman in figure 2 shows that the values for their short wavelength group are too small, as would be the case if extraneous radiation were superimposed on the absorption lines. From this plot their values have been corrected and adjusted to the CB scale. The corrected values are entered in table 2 under the heading VNS.

\section{e. Valters and Startsev [1964]}

Valters and Startsev determined oscillator strengths for 89 lines of $\mathrm{Fe}$ I with lower levels $a^{5} \mathrm{~F}$ and $a^{5} \mathrm{D}$ from the anomalous dispersion of iron vapor in a furnace. The results cover the same wavelength range as the absorption results of Valters, Nikonova, and Startsev, but a plot of the ratio of their values to those of Corliss and Bozman in figure 2 does not show any dependence on wavelength. This result would be expected because of the nature of the anomalous dispersion method and indeed was also noted in the case of Penkin's results. Valters and Startsev's values have been adjusted to the scale of Corliss and Bozman by subtracting 3.20 and then entered into table 2 under the heading VS.

Figure 2 shows how systematic errors of measurement can seriously override random errors in oscillator strength work.

\subsection{Data of Crosswhite}

For more than 20 years a program of intensity measurements in atomic and molecular spectra has been conducted at The Johns Hopkins University spectroscopy laboratory by the late Prof. G. H. Dieke. Many of the measurements, particularly those in the spectrum of iron, were made by Dr. H. M. Crosswhite. In 1950 Crosswhite published the results of his intensity measurements in a $2.2 \mathrm{~A}$ iron arc. These measurements, which include 1064 lines in the region from 3147 to $5659 \AA$, have been reduced to oscillator strengths by Corliss and Warner (1964).

In 1958 Crosswhite published a monograph on Fe I which included not only his measurements in the $2.2 \mathrm{~A}$ arc but also measurements made in a $1 \mathrm{~A}$ arc and in a hollow cathode. Some of these measurements have also been published in the American Institute of Physics Handbook, pp. 7-89 through 7-102. The observations from the $1 \mathrm{~A}$ arc are not as numerous as those from the other sources, nor are they any more accurate, so we have not attempted to derive oscillator strengths from them.

With the hollow cathode, however, he has measured more than 1000 lines in the region from 3200 to $4150 \AA$, about 400 between 2450 and $3200 \AA$ and about 700 lines between 4150 and $8000 \AA$. Above $3150 \AA$ Crosswhite made his sensitivity calibration with a standard tungsten ribbon-filament lamp calibrated at the National Bureau of Standards; between 2700 and $3100 \AA$ he made an indirect calibration from a study of selfabsorption and below $2700 \AA$ he extrapolated. A plot of the ratio of the hollow cathode intensities to those from Meggers, Corliss, and Scribner [1961] shows that, with the exception of a discontinuity at $3730 \AA$, the ratio is constant from 3200 to $8400 \AA$. Below $3200 \AA$ the ratio becomes very irregular and the scatter increases. The discontinuity at $3730 \AA$ amounts to 0.35 in the $\log$, which is the same as that found by Corliss and Warner in Crosswhite's $2.2 \mathrm{~A}$ arc data. Figure 3 shows the plot in the region 3000 to $4250 \AA$ for lines whose upper levels lie between 46 and $53 \mathrm{kK}$ (kilokaysers). Comparison with the similar plots for the hook method data, which should be free of wavelength dependent errors, indicates that the discontinuity must lie in Crosswhite's intensities. We corrected the discontinuity before further discussion of the data.

Crosswhite's iron hollow cathode tube was filled with neon to a pressure of $3.5 \mathrm{~mm} \mathrm{Hg}$ and operated at $90 \mathrm{~mA}$. The strong lines were measured photoelectrically. "The much larger group of weaker lines is more conveniently measured photographically. Although the intensity range encountered with the photoelectric measurements was almost 10000:1, the photographic one is much less and can be encompassed by the use of a few neutral screens, with reference being made to the photoelectric values for purposes of plate calibration and standardization." Crosswhite [1958] goes on to say, "In spite of difficulties it appears that, at least within a given multiplet, transition probability determinations are possible. Comparison between different multiplets is more cumbersome than in an equilibrium case, but not impossible. It certainly seems worthwhile to make some effort to utilize the great stability of the intensities which are reproducible to better than 1 percent for a particular experimental arrangement."

It does indeed seem worth an effort to derive oscillator strengths from Crosswhite's measurements of hollow cathode intensities. To carry out this reduction, it is first necessary to determine the relative occupation numbers of the upper energy level of each measured transition. In an equilibrium source these numbers are determined by measuring the temperature of the source and calculating the occupation numbers with Boltzmann's exponential law. At pressures as low as $3.5 \mathrm{~mm} \mathrm{Hg}$ it is generally thought that a source is no longer in LTE and that the population distribution can no longer be described as simply

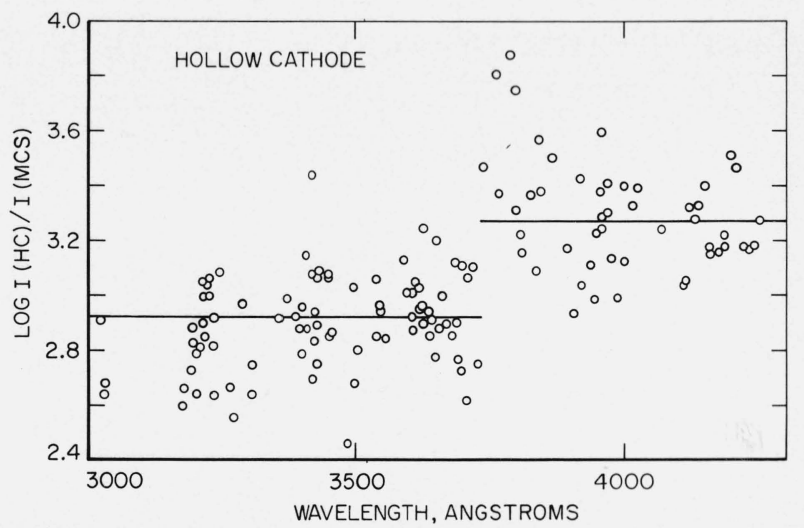

FIGURE 3. Comparison of the intensity measurements of Crosswhite [1958] in the hollow cathode with those of Meggers, Corliss, and Scribner [1961] in a copper arc for lines of $\mathrm{Fe} I$ whose upper levels lie between 46 and $53 \mathrm{kK}$; wavelength range 3000 to $4500 \AA$. 
exponential. To test this assumption we calculated the relative occupation number of each upper level for a selected group of lines in Crosswhite's hollow cathode list. These are the lines between 3200 and $9000 \AA$ for which transition probabilities are listed in Corliss and Bozman. To calculate the relative occupation numbers of the upper levels of the iron atoms in Crosswhite's hollow cathode we recall that

$$
I=h \nu N_{n} A_{n m} \text {, so that } I \lambda / A_{n m} \sim N_{n}
$$

or

$$
\frac{I \lambda}{g_{n} A_{n m}} \sim \frac{N_{n}}{g_{n}}
$$

Boltzmann's exponential law states that

$$
\frac{N_{n}}{g_{n}}=\frac{N_{0}}{g_{0}} e^{-E / k T}
$$

where the subscript 0 refers to the ground state. Now if we plot $\log I \lambda / g A$ (or its reciprocal) versus $E$, the value of the upper energy level, we will see graphically the population distribution amongst the levels of the atom. In figure 4 we have plotted $\log g A / I \lambda$ for every line between 3200 and $9000 \AA$ which appears in both Corliss and Bozman (from which the values of $g A$ are taken) and Crosswhite's hollow cathode list (from which the values of $I$ are taken). In most cases each point represents the mean value for 2 to 4 lines. Figure 4 represents the population distribution amongst energy levels of iron atoms in Crosswhite's hollow cathode for every upper level listed in Corliss and Bozman. The dashed line represents an equilibrium distribution at a temperature of $4560{ }^{\circ} \mathrm{K}$. The departures of the individual points from an equilibrium line seem to support an abandonment of the notion of LTE

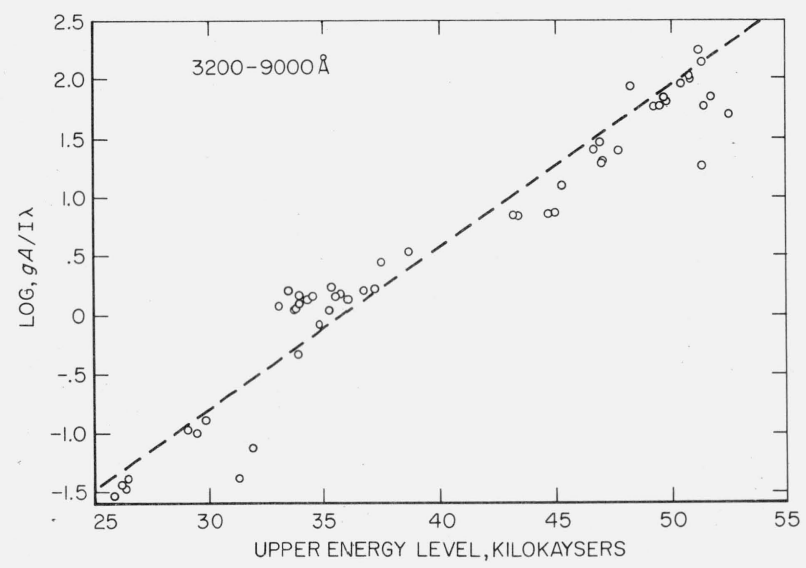

FIGURE 4. Population distribution (reciprocal) amongst energy levels of iron atoms in Crosswhite's [1958] hollow cathode for every upper level listed in Corliss and Bozman [1962]. for the most accurate reduction of Crosswhite's hollow cathode intensity measurements to oscillator strengths.

The data shown in figure 4 permit determination of occupation numbers for the iron atoms in the hollow cathode for only about 50 upper energy levels. To obtain a more complete specification of the population distribution, we repeated the process using the oscillator strengths for 2000 lines of $\mathrm{Fe}$ I given by Corliss and Warner. This allowed us to find occupation numbers for 323 levels with an average number of determinations per level of about 4 . With the value of $N / g$ for each of these levels known, we calculated $\log g f$ from each value of hollow cathode intensity using the formula $\log g f=\log I_{h c}+3 \log \lambda-\log N / g$ -15.82 , where the value of the numerical constant depends on the scale of $I$. This determination makes no assumption about the population distribution among the upper levels of the iron atoms in the hollow cathode discharge. The values derived are entered in column 9 of the table 3 under the heading HC.

Inspection of table 3 reveals that a number of values of $\log g f$ derived from the hollow cathode intensities are in rather wide disagreement with the other tabulated values. We used the values of $\log I_{2}$ from table XIII of Crosswhite [1958] to derive the values of log $g f$. Comparison of these $\log I_{2}$ values with those given in the American Institute of Physics Handbook and with the photoelectric traces of hollow cathode spectra reproduced in both publications indicates that a number of these discrepant values are certainly due to misprints. For this reason, discrepant hollow cathode values have been freely ignored in arriving at the "best" values given in table 3 .

\subsection{Data of Corliss and Bozman}

In 1963 Corliss and Bozman published NBS Monograph 53, "Experimental Transition Probabilities for Spectral Lines of Seventy Elements," which is derived from the intensity measurements of Meggers, Corliss, and Scribner [1961]. They reported 663 oscillator strengths for $\mathrm{Fe}$ I of which about 450 lie below $4150 \AA$. For a number of reasons their scale is suitable to use in a compilation such as ours. The number of values reported is so large that there are numerous lines in common with every published list. This simplifies the problem of putting all measurements onto a common scale. Their absolute scale has been shown by Allen and Corliss [1963] to be reasonably accurate for all the neutral atoms in the iron group. They concluded that the CB scale may be high by 0.05 in log $g f$, on the average. We discuss the accuracy of the $\mathrm{CB}$ absolute scale for $\mathrm{Fe} \mathrm{I}$ in particular in section 5 of this paper. The temperature of their light source was determined with an accuracy of \pm 2 percent. The precision of their measurements, while not high, is accurately known and compares well with that of most other published sets of data for Fe I. The comparisons shown in figure 2 indicate that the intensity scale of Meggers, Corliss, and Scribner is correctly calibrated as a function of wavelength from 2700 to $4500 \AA$. 


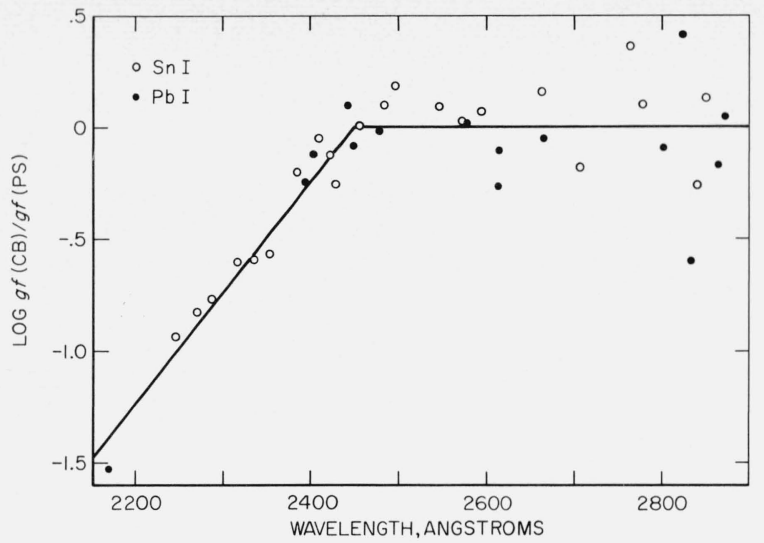

FigURE 5. Ratio of the gf-values of Corliss and Bozman [1962] to those of Penkin and Slavenas [1963] (normalized to unity above $2450 \AA$ A) between 2150 and $3000 \AA$.

However, the intensity scale of Meggers, Corliss, and Scribner is subject to a serious systematic error below $2500 \AA$, where it becomes progressively too small. The origin of this error lies in the notorious lack of any reliable energy calibration procedure for short wavelengths. Soon after the publication of NBS Monograph 53, Penkin and Slavenas [1963] published oscillator strengths for lines of tin and lead which extended down to $2170 \AA$. They used the hook method, which does not require an intensity calibration of any sort. A plot of the ratio of their values to those of Corliss and Bozman (normalized to unity between 2450 and $3000 \AA$ ) is shown in figure 5 . The figure shows that the ratio is constant between 2450 and $3000 \AA$ but that the CB scale declines by a factor of 30 between 2450 and $2150 \AA$. This correction has been applied to the $\mathrm{CB}$ values reported in column 8 of table 3 .

There are some independent data below $2500 \AA$ that support the correction derived from the work of Penkin and Slavenas. The relative oscillator strengths for Co II calculated by Gruzdev [1962] with the method of intermediate coupling give rise to a correction curve in substantial agreement with that of figure 5 . We have, however, preferred to base our calibration on experimental values. Measurement of intensities of Sn I lines in chemiluminescent flames led Gilbert [1963] to the conclusion that the values of Meggers, Corliss, and Scribner [1961] decreased by a factor of 50 between 2500 and 2100 A. This agrees with our adopted correction.

\subsection{Data of Margoshes and Scribner}

Margoshes and Scribner [1963] determined relative oscillator strengths for 105 lines of Fe I between 2900 and $4150 \AA$ using a gas stabilized arc (a form of plasma jet) as an emission source. All of these lines had been measured previously, but by other methods. Figure 5 in their paper shows the ratio of their $g f$-values to those of Corliss and Bozman. There appears to be a slight trend of the ratio with wavelength which hardly exceeds the uncertainty of the measurements and we have made no correction. The absolute value of their scale has been altered by adding 0.23 to their values of $\log g f$, thereby putting their measurements onto the common scale of table 3 . Their values are listed in column 12 under MS.

\subsection{Data of Corliss and Warner}

In 1964 Corliss and Warner published a compilation of oscillator strengths for $\mathrm{Fe} I$ that emphasized the visible and infrared regions of the spectrum. In addition to their own measurements in the visible and infrared, they included all data published prior to 1963, with the exception of Sobolev [1943] and Parchevsky and Penkin [1954] which were not available to them, and the hollow cathode data of Crosswhite [1958] (v. sec. 2.3). These data included King and King [1938], Carter [1949], Crosswhite [1950], Allen and Asaad [1957], and Corliss and Bozman [1962] longward of $3100 \AA$. The values of Mitrofanova [1952]; Aarts, Harting, and Bakker [1954]; and Hefferlin [1959] were discussed but not incorporated into their "best" value.

The "best" value of Corliss and Warner is entered in column 8 under CW. As in the tabulation of the Russians' measurements, a superscript following the value indicates the number of entries contributing to their "best" value and is used as a weighting factor in arriving at the "best" value in column 13 . The CW values include and supersede those of Corliss and Bozman at wavelengths above $3100 \AA$. Since the CW values end at $3100 \AA$, the $C B$ values are tabulated in the same column at shorter wavelengths.

\subsection{Data of King, Olsen, and Corliss}

In 1965 King, Olsen, and Corliss published oscillator strengths for $300 \mathrm{Fe}$ I lines between 2500 and $3200 \AA$. They were calculated from the equivalent widths of lines absorbed from the continuum of a high pressure Xenon lamp when its radiation was passed through iron vapor in a graphite-tube electric furnace. Scattered light was controlled with a Corex red-purple filter. To avoid the flat portion of the curve-of-growth in the reduction of the data, only the faintest lines in each exposure were measured. Since the lines observed cover a very large range of intensities, a great many spectrograms would be required to put all of the lines on the same scale of intensity. Although 86 separate exposures were made, it was nevertheless necessary to tie some of them together with supplementary data. For this purpose the oscillator strengths of Corliss and Bozman were used. This procedure should not introduce any wavelength dependent error or energy level dependent error, even if such an error were present in the CB data. The only error which could be introduced from the supplementary data would be an 
intensity dependent error. Such an error is most unlikely.

In figure 6 is plotted the ratio of oscillator strengths from KOC to those from $\mathrm{CB}$ as a function of upper energy level in kilokaysers. With the exception of two points at about $60 \mathrm{kK}$, there is only a very slight dependence of ratio on upper energy level. Qualitatively, this dependence is in the sense that the KOC temperature scale is slightly smaller than the CB scale which is in accord with results found earlier by Corliss [1962]. The same ratio is plotted as a function of wavelength in figure 7 . The ratio seems to be independent of wavelength, with the possible exception of the region from 3100 to $3200 \AA$. The remarkable departure of the two high level lines at 2542 and 2543 $\AA$ from the general trend of the plots is unexplained. The values from this paper are entered in column 11 of - table 3 under KOC.

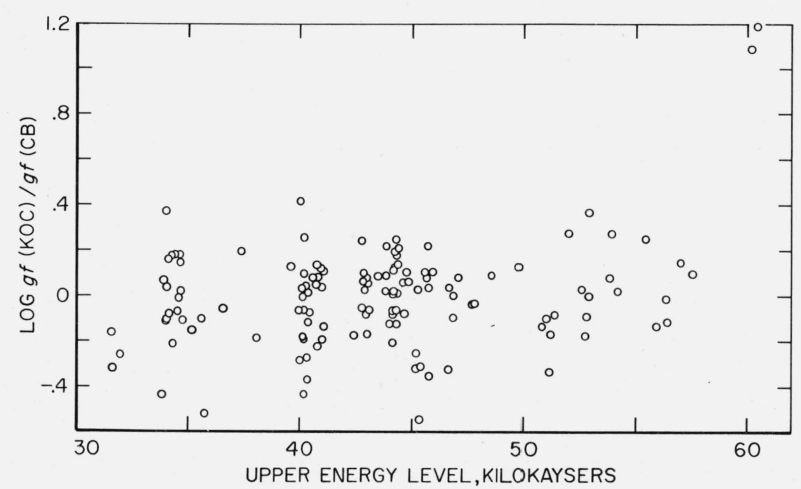

Figure 6. Ratio of gf-values from King, Olsen, and Corliss [1965] to those from Corliss and Bozman [1962] as a function of upper energy level.

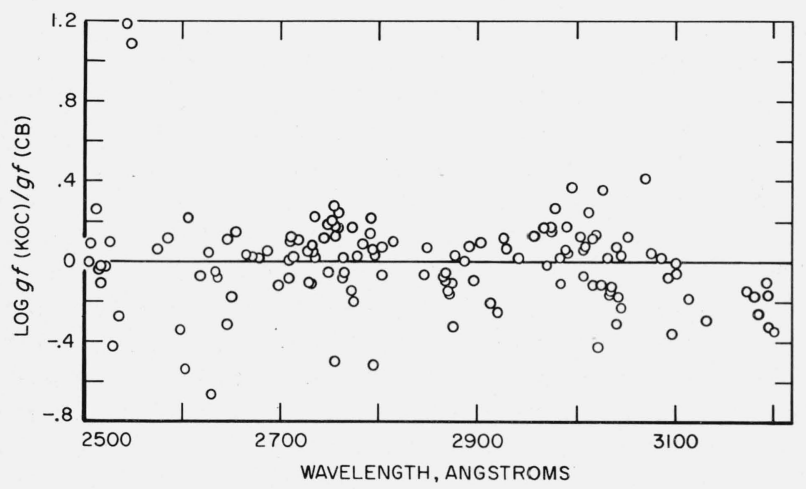

Figure 7. Ratio of gf-values from King, Olsen, and Corliss [1965] to those from Corliss and Bozman [1962] as a function of wavelength; 2500 to $3200 \AA$.

\section{New Observations}

\subsection{Data of Warner From ULO}

In the region from 2500 to $4150 \AA$, new observations have been made at the University of London Observatory using both free-burning arcs and sparks. A d-c arc was maintained between two $5 \mathrm{~mm}$ diam iron rods. These electrodes were obtained from Messrs. Johnson Matthey and Co. and contain only one or two parts per million of spectroscopically conspicuous impurities. A current of $1 \mathrm{~A}$ was drawn from a constant-current source. Such a small current, although necessitating longer exposure times than is usual, has the advantage of considerably reducing the amount of self absorption in strong lines.

The spark source, operated at $10,000 \mathrm{~V}$, was produced between pure iron electrodes in an argon atmosphere at normal pressure. The average current was 1.3 A, which caused the electrodes to glow white hot as in a normal arc. This resulted in a very stable, nonwandering spark. The argon atmosphere removed troublesome $\mathrm{O}$ II, $\mathrm{N}$ II, $\mathrm{O}_{2}^{+}$, and $\mathrm{N}_{2}^{+}$emissions that occur in a spark in air. A few Ar I and Ar II lines are observed. The argon atmosphere also reduced the self-absorption in strong lines to almost negligible amounts. A disadvantage of the spark was that exposure times up to $75 \mathrm{hr}$ were necessary to record the spectral region below $3000 \AA$. Also, in this region Fe II lines interfered with many neutral lines.

The light from the sources was imaged by means of two quartz lenses onto the grating of the stigmatic Ebert spectrograph in the laboratory of the University of London Observatory. This spectrograph of $3.4 \mathrm{~m}$ focal length, has a 15000 lines per inch grating giving a dispersion of $4.75 \mathrm{~A} / \mathrm{mm}$ in the first order. A total wavelength region of $2400 \AA$ can be recorded in one exposure. Ilford HP3 plates were used throughout the present investigation. These were brushed continuously during development.

On all plates a spectrum of the iron arc through a calibrated step wedge (placed at the spectrograph slit) was obtained. All arc and spark spectra were traced with the recording microphotometer at the Royal Greenwich Observatory. From the step wedge spectra, photographic calibration curves were drawn for each plate. Line and continuum readings from the charts were converted into intensities with the aid of these characteristic curves. The continuum intensity was subtracted from the apparent line intensity to give the true line intensity.

In general at least three independent intensity measurements were made on each line. Over most of the intensity range the plate-to-plate interagreement of line intensities when placed on a uniform scale, was $\sim 0.03$ dex. $( \pm 7 \%)$. In the arc spectra all strong lines were rejected to avoid effects of self-reversal.

Our intensity measurements in a range of $200 \AA$ near $4100 \AA$ were compared with the $\mathrm{Fe}$ i $f$-values given by Corliss and Warner in the manner described by Corliss and Warner and the excitation temperatures 
for the two sources derived. For the $1 \mathrm{~A} \mathrm{~d}$-c arc a value of $4630 \pm 70^{\circ} \mathrm{K}$ was found. In figure 8 we show the graph from which the excitation temperature of the spark was determined. It can be seen that for upper energy levels where $\mathrm{E}<48 \mathrm{kK}$ the population of levels in our spark source is closely Boltzmannian. The derived temperature is $6170 \pm 30{ }^{\circ} \mathrm{K}$. The departure from linearity in figure 8 for $\mathrm{E}>48 \mathrm{kK}$ is a reflection of the normalization function applied to the Corliss and Warner $f$-values, and will be discussed later.

With the above determined excitation temperatures we can calculate from the $\mathrm{CB}$ (and $\mathrm{CW}$ ) Fe I $f$-values, a theoretical line intensity for all lines in common with the present work (with $E<48 \mathrm{kK}$ ):

$$
\log I_{\mathrm{CB}} \lambda^{3}=\log g f_{\mathrm{CB}}-\frac{625 E}{T_{\mathrm{ex}}}
$$

where $E$ is in kK. We then plot $\log I_{\mathrm{CB}} \lambda^{3}-\log I_{\mathrm{W}}$ as a function of $\lambda$. This gives the wavelength calibration for the plate (i.e., determines the combined effects of plate sensitivity and spectrograph transmission) and at the same time takes care of the $\lambda^{3}$ factor. Using this graph we correct all intensities measured in the present work and obtain values of $\log I_{\mathrm{W}} \lambda^{3}$ on an arbitrary scale. Relative $g f$ values for all lines were then calculated from

$$
\log g f_{\mathrm{rel}}=\log I_{\mathrm{W}} \lambda^{3}+\frac{625 E}{T_{\mathrm{ex}}} .
$$

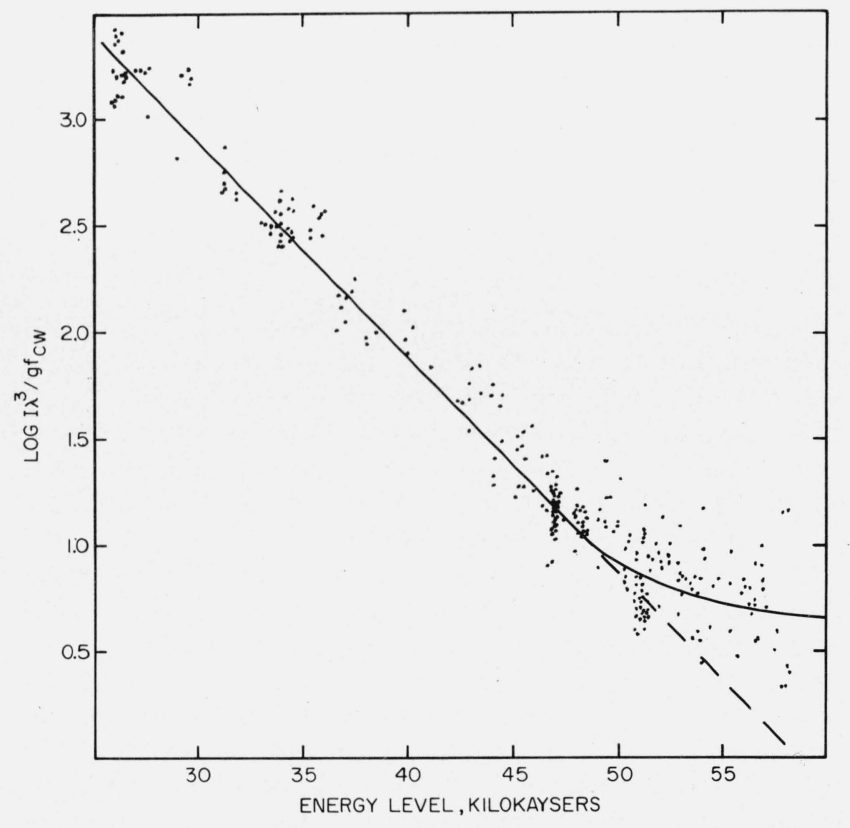

Figure 8. Log ratio of intensity $\times \lambda^{3}$ to gf-value of Corliss and Warner [1964] for Fe I lines in the iron spark plotted versus upper energy level.
These relative values were corrected for departure from a Boltzmann distribution and adjusted to the absolute scale by plotting this $\log g f_{\text {rel }}-\log g f_{\mathrm{CB} \text { or } \mathrm{CW}}$ versus $E$ for lines in common with $\mathrm{CB}$ or $\mathrm{CW}$ and applying the indicated correction to every line. The values are listed in columns 6 and 7 of table 3 .

\subsection{Data of Corliss From NBS}

In the region below $2500 \AA$ a new set of observations has been made at the National Bureau of Standards and is reported in column 1 of table 3. Since this region is densely populated with lines of the first and second spectrum of iron, observations were made in the third order of a 30000 lines per inch grating mounted. in parallel light. The second order was eliminated with a chlorine filter. A step sector wheel with sector openings in the ratio of two was mounted at the slit and reduced the intensity by that ratio in eight successive steps along the length of the slit image. An achromatic lens at the slit formed an image of the light source on the grating and permitted uniform illumination of the slit (in the absence of the sector wheel).

The light source was a $1 \mathrm{~A}$ arc between a 7/32 in. diam rod of commercially pure iron as the lower anode and a pointed $3 / 8$ in. graphite upper cathode. Exposure times were about an hour on Kodak 103a-0 plates. Three sets of plates were measured.

The intensities were estimated by visual interpolation between steps in the sectored spectrograms. With these intensities and $g f$-values from Corliss and Bozman for the range 2450 to $2540 \AA$, a plot of log $I \lambda^{3} / g f$ versus upper energy level was prepared. From this plot preliminary values of $\log g f$ for all the measured lines were calculated. Finally, a correction to be applied to all of these values below 2450 A was prepared by plotting the ratio of these preliminary values to the corrected $\mathrm{CB}$ values from column 8 of table 3. After correction, the new values were entered in column 6 of table 3 . To make certain that the new values were not affected by self-absorption, the ratio of the new values to those in column 8 were plotted versus the corresponding intensity from Meggers, Corliss, and Scribner for lines between 2450 and $2540 \AA$. A horizontal plot showed that at $1 \mathrm{~A}$ these lines were not self-absorbed.

\section{Results}

The results of the compilations and measurements discussed in sections 2 and 3 are presented in table 3. The wavelengths are taken in order of preference from Edlén [1955]; from Russell and Moore [1944]; from Kiess, Rubin, and Moore [1961]; or, in a few cases, are calculated from the term combinations. The classifications are taken from the same papers and the numerical values of the energy levels are given to the nearest kayser in columns 2 and 3 . The lower excitation potential in electron volts has been calculated by multiplying the lower energy level in column 2 by 0.00012398 and the result, rounded off 
to two decimal places, is entered in column 4 . The multiplet numbers in column 5 are those assigned by Moore [1945, 1952] in her Multiplet Tables.

The recommended or "best" value of $\log g f$ given in column 13 is, in general, the unweighted mean of the individual values of $\log g f$ in the previous seven columns. However the $\mathrm{CW}$ and $\mathrm{R}$ values have been weighted according to the number of contributing values in Corliss and Warner or in table 2 and the weighting number is indicated as a superscript. In a few cases, where there was good reason, individual values were given a weight of zero. If the maximum and minimum values for any line differ by more than 0.4 , no best value is recommended unless there is a value obviously discrepant.

$\log g f \lambda$ and $\log g A / \lambda$ have been computed from the best value and are given in the last two columns. $\log g f \lambda$ is useful in dealing with curves of growth in absorption spectra and stellar spectra and $\log g A / \lambda$ in determining temperatures from intensity measurements in emission spectra. It should be noted also that the line strength, $S$, is equal to $g f \lambda / 304$, or $\log S=\log g f \lambda-2.48$.

\section{Discussion of the Absolute Scale}

In this section we discuss the various attempts to make absolute determinations of oscillator strengths for lines of $\mathrm{Fe} \mathrm{I}$ and try to decide if a scale more accurate than that used in this paper can be recommended.

A number of determinations of the oscillator strength of the resonance line of Fe I at $3719.937 \AA$ by various atomic beam experiments have been reported over the past 15 years. They are listed below, with the value of $\log g f$.

Kopfermann and Wessel [1951]....... - 0.41

Ziock [1957].......................... -0.38

Bell, Davis, King, and Routly [1958]... - 0.54

Ottinger and Ziock [1961]............ -0.50

The mean value here is -0.46 , which is 0.14 less than the value reported for that line in our table.

In 1942, R. B. King made absolute determinations of the oscillator strengths of 12 lines of $\mathrm{Fe}$ I between 3700 and $3900 \AA$ by measuring the total absorption in a furnace. Bell, Davis, King, and Routly [1958] revised those values on the basis of new vapor pressure data. The revised values for these 12 lines are on a scale of $\log g f$ which is 0.25 less than that of our table.

In their work on the abundances of the elements in the solar atmosphere, Goldberg, Müller, and Aller [1960] calculated absolute values of $\log g f$ for Fe I lines of the $3 d^{7} 4 s-3 d^{7} 4 p$ transition array from the $f$-sum rule, assuming $f_{T}=1.0$ The array has 45 lines in the range 3585 to $4150 \AA$. This $f$-sum scale is 0.06 less than the scale of our table.

With a somewhat different formulation of the $f$-sum rule, assuming $f_{T}=0.7$, Allen [1960] adjusted a large number of relative oscillator strengths for $\mathrm{Fe} I$ to an absolute scale. A comparison of that scale with the scale of Corliss and Bozman made by Allen and Corliss [1963] leads to the conclusion that Allen's $f$-sum scale is 0.20 less than our present scale.

In an interesting experiment reported to the Aeronautical Systems Division, AFSC in 1965, Karstensen and Richter of the University of Kiel measured the lifetimes of the $z^{3} \mathrm{~F}_{4}$ and $z^{3} \mathrm{D}_{1}$ levels in Fe I. They also calculated them from the oscillator strengths of Corliss and Warner [1964] which are reported on the present scale. The lifetimes in nanoseconds are quoted below from their report.

$\begin{array}{lcr} & z^{3} \mathbf{F}_{4} & z^{3} \mathrm{D}_{1} \\ \text { Karstensen and Richter......... } & 220 \pm 70 & 100 \pm 25 \\ \text { Corliss and Warner............. } & 240 \pm 120 & 80 \pm 40\end{array}$

There does not appear to be any significant difference between the two sets of determinations.

Paul L. Byard [1966] of the Ohio State University Department of Astronomy has been making oscillator strength measurements in Fe I using a luminous shock tube. Preliminary results obtained for a group of lines in the red part of the spectrum show agreement with the values of Corliss and Bozman within the experimental uncertainties.

From a discussion such as this we cannot hope to arrive at an exact correction to be applied to our scale. It is obvious that the absolute scale is still somewhat uncertain. The mean correction obtained from the six determinations mentioned above is -0.11 with a standard deviation of this mean amounting to 0.04 . At the present time therefore, it would seem that the scale used in this paper is high by about 0.1 in $\log g f$. In order to keep the scale in this paper uniform with that of our previous work in the visible and infrared parts of the spectrum, we have not applied the correction here. Furthermore, there is little doubt that this figure is subject to some change as more data on the absolute scale of oscillator strengths for Fe I is accumulated.

\section{Conclusion}

In this paper numerous oscillator strength measurements, both new and old, for 2000 lines of Fe I have been assembled, adjusted to the same scale, and best values for most of the lines suggested. The scale on which they are presented is the same as that used by Corliss and Bozman [1962] for Fe I and by Corliss and Warner [1964]. The values in this paper supersede the values below 4151 $\AA$ in Corliss and Warner [1964] and, together with the remaining 1349 lines in that paper, provide a total of 3349 lines of $\mathrm{Fe}$ I for which more or less accurate values of oscillator strengths are known. These are more than three-quarters of the classified lines of $\mathrm{Fe}$ I which have been observed in the laboratory.

The accuracy of the best values of $\log g f$ in table 3, taken as relative values, will vary greatly from line to line, depending on the number and origin of the obser- 
vations contributing to it. The standard deviation of the new observations should be about the same as those of Corliss and Warner [1964] which were stated to be about 0.13. Graphical representation of the overall scatter encountered in the construction of curves of growth with those values are given by Bell and Rodgers [1965] for stellar atmospheres and for the solar atmosphere by Goldberg, Kopp, and Dupree [1964]; by Warner [1964]; and by Aller, O'Mara, and Little [1964].

The absolute scale on which these values is reported appears to be about 25 percent higher than the best absolute scale for $\mathrm{Fe}$ I that can be established at present. The uncertainty in the best absolute scale, on the basis of the consistency amongst the various sets of determinations considered above seems to be about 11 percent (standard deviation of the mean for six sets). Therefore, for work requiring the best present estimate of the absolute scale, we suggest subtracting 0.10 from our reported values of $\log g f$. Improved knowledge of this correction can be expected in the near future.

Warner thanks the Astronomer Royal for the use of the recording microphotometer at the Royal Greenwich Observatory. He is also grateful to E. L. G. Bowell and K. J. H. Phillips for carrying out some of the numerical work. He was supported by a Research Grant from the Department of Scientific and Industrial Research. We also thank Ruth Peterson for her conscientious work in making the tables and putting them on punched cards, and W. R. Bozman who wrote a program for computing several quantities in the tables.

\section{References}

Aarts, J., Harting, D., and Bakker, C. J. (1954), Physica 20, 1250. Allen, C. W. (1960), Monthly Notices Roy. Astron. Soc. 121, 299. Allen, C. W. and Asaad, A. S. (1957), Monthly Notices Roy. Astron. Soc. 117,36 .

Allen, C. W. and Corliss C. H. (1963), Monthly Notices Roy. Astron. Soc. 126, 37.

Aller, L. H., O'Mara, B. J., and Little, S. J. (1964), Proc. Nat. Acad. Sci. 51, 1238.

American Institute of Physics Handbook (McGraw-Hill Book Company, Inc., New York, 1957).

Bell, G. D., Davis, M. H., King, R. B., and Routly, P. M. (1958), Ap. J. 127, 775.

Bell, R. A. and Rodgers, A. W. (1965), Monthly Notices Roy. Astron. Soc. 129, 127.

Byard, P. L. (1966) (private communication).

Carter, W. W. (1949), Phys. Rev. 76, 962.

Corliss, C. H. (1962), J. Res. NBS 66A (Phys. and Chem.) No. 1, 5.
Corliss, C. H. and Bozman, W. R. (1962), Experimental transition probabilities for spectral lines of seventy elements, NBS Mono. No. 53 [W ashington: Government Printing Office].

Corliss, C. H. and Warner, B. (1964), Ap. J. Supplement Series 8, 395, No. 83.

Crosswhite, H. M. (1950), Spectrochimica Acta 4, 122

Crosswhite, H. M. (1958), The Spectrum of Iron I, Johns Hopkins Spectroscopic Report No. 13, Baltimore.

Edlén B. (1955), Trans. I. A. U. 9, 220.

Gilbert, P. T. (1963), Proc. of the Xth Colloq. Spectros. Internat. p. 207.

Goldberg, L., Kopp, R. A., and Dupree, A. K. (1964), Ap. J. 140, 707.

Goldberg, L., Müller, E. A., and Aller, L. H. (1960), Ap. J. Suppl. Series No. 45, 87.

Gottschalk, W. M. (1948), Ap. J. 108, 326.

Gruzdev, P. F. (1962), Opt. Spectry, 13, 169

Hefferlin, R. (1959), J. Opt. Soc. Am. 49, 680, 948.

Kartensen, F. and Richter, J. (1965), New Method of Measuring Transition Probabilities, AF EOAR Grant 63-110.

Kiess, C. C., Rubin, V. C., and Moore, C. E. (1961), J. Res. NBS 65A (Phys. and Chem.) No. 1, 1.

King, R. B. (1942), Ap. J. 87, 24.

King, R. B. and King, A. S. (1938), Ap. J. 87, 24.

King, R. B., Olsen, K. H., and Corliss, C. H. (1965), Ap. J. 141, 354.

Koperfmann, H. and Wessel, G. (1951), Zeits. f. Physik 130, 100.

Margoshes, M. and Scribner, B. F. (1963), J. Res. NBS 67A (Phys. and Chem.) No. 6, 561 .

Meggers, W. F., Corliss, C. H., and Scribner, B. J. (1961), Tables of Spectral-line intensities, NBS Mono. No. 32 [Washington: Government Printing Office].

Mitrofanova, L. A. (1952), Izv. Astro. Obs. Pulkovo 19, 81.

Moore, C. E. (1945), A Multiplet Table of Astrophysical Interest, Contr. Princeton Univ. Obs. No. 20.

Moore, C. E. (1952), An ultraviolet multiplet table, NBS Circ. 488 , Sec. 2.

Morzova, N. G., Startsev, G. P., and Frish, M. S. (1960), Physical Problems in Spectroscopy. Proc. of the XIIIth All-Union Conf. on Spectroscopy, Leningrad, 4-12 July 1960. Vol. 1, p. 45, Moscow, 1962

Ottinger, C. and Ziock, K. (1961), Zeits, Naturforsch 16a, 720.

Parchevskii, G. F. and Penkin, N. P. [1954], Vestnik Leningrad State University 11, 113. Translated by J. Tech. in Astron. Papers Trans. from the Russian, No. II, (1964), Smithsonian Astrophys. Obs., Cambridge, Mass.

Penkin, N. P. (1964), J. Quant. Spectros, Radiative Transfer 4, 41. Translated by J. Tech. in Astron. Papers Trans. from the Russian, No. II (1964), Smithsonian Astrophys. Obs., Cambridge, Mass.

Penkin, N. P. and Slavenas, I. Y. Y. (1963), Opt. Spectry. 15, 83.

Prokofiev, V. K., Nikonova, E. I. Gruzdev, F. P., and Frish, M. S (1964), Bull. Crimean Astrophys. Obs. 31, 281.

Russell, H. N. and Moore, C. E. (1944), Trans. Am. Phil. Soc. 34, (Part II) p. 113.

Shore, B. W. (1965), Ap. J. 142, 94.

Sobolev, N. N. (1943), J. Experimental and Theoretical Physics $13,131$.

Valters, A. K. Nikonova, E. I., and Startsev, G. P. (1964), Opt. Spectry. 16, 393 (English transl.).

Valters, A. K., and Startsev, G. P. (1964), Opt. Spectry. 17, 262 (English transl.).

van Milaan, J. B. (1926), Zeits. f. Physik 38, 422.

Warner, B. (1964), Monthly Notices Roy. Astron. Soc. 127, 413.

Warner, B. (1966), Memoirs Roy. Astron. Soc. (in press).

Ziock, K. (1957), Zeits. f. Physik 147, 99. 
TABLE 3.-Oscillator Strengths for Ultraviolet Lines of Fe I

Wave-

Energy Levels Low

$\mathrm{K}$ Volts

Mult.

Arc $\quad$ Spk $\quad \mathrm{CB} \quad \mathrm{HC}$
$-\mathrm{Log} g f$

No.

2084.122
2157.795

2164.550

2166.769

2171.298

2178.080

2178.120

2186.483

2186.893

2187.195

2191.836

2196.043

2200.370

2200.723

2248.858

2250.791

2259.511

2264.389

2265.055

2266.903

2267.085

2267.465

2269.099

2270.863

2271.781

2272.070

2272.816

2275.189

2276.026

2277.098

2277.672

2279.922

2280.222

2283.304

2283.656

2284.086

2287.250

2289.032

2290.546

2291.122

2292.525

2294.406

2296.928

2297.788

2298.170

2298.657

2299.221

2300.140

2301.685

2303.422

2303.582

2309.000

2313.105

2320.358

2341.575

2350.408

2355.327

2356.196

2369.457

2371.431 $\begin{array}{lll}0-47967 & 0.00 & \text { UV } 33\end{array}$

$\begin{array}{lll}416-46745 & 0.05 & \text { UV } 24\end{array}$

$\begin{array}{rlll}704-46889 & 0.09 & \text { UV } 24\end{array}$

$0-46137$

$704-46745$

0.00

UV 21

UV 24

$416-46314$

$704-46601$

$416-46137$

$888-46601$

$704-46410$

$704-46314$

$888-46410$

$978-46410$

$888-46314$

$6928-51381$

0.05

0.09

U V 21

UV 22

UV 21

0.11

UV 22

U V 21

$0.09 \quad$ U V 21

0.11

0.12

UV 21

U V 21

0.11 UV 21

0.86

U V 70

$0-44415$

$0-44244$

$6928-51077$

$416-44551$

0.00
0.00

UV 16

UV 15

0.05

UV 16

U V 70

$416-44512$

$6928-51017$

$704-44761$

$0-44023$

$7377-51381$

0.05

UV 17

0.86 UV 70

0.09 UV 16

0.00 U V 15

0.91

U V 70

$416-44415 \quad 0.05$

$888-44827$

$0-43923$

0.91

UV 16

0.11

0.00

UV 71

UV 16

U V 14

UV 71

$7728-51619$

$704-44551$

$7986-51828$

$978-44761$

$888-44664$

0.96 U V 70

0.99 UV 70

0.12

U V 16

U V 16

416-44184

0.05

$704-44411$

0.09

$\begin{array}{lll}\text { U V } 14 & 14\end{array}$

UV 14

1.01 U V 70

0.99

$7986-51630$

$7728-51361$

0.96

U V 71

U V 71

$416-44023$

$888-44459$

$888-44411$

$416-43923$

0.05

U V 15

0.11 U V 14

U V 14

0.05

U V 14

$0-43500$

U V 14

$888-44378$

$704-44184$

$704-44166$

0.11

0.09

0.09

U V 15

UV 14

U V 15

$978-44411$

0.12

U V 14

U V 15

$888-44285$

$888-44184$

$704-43923$

$416-43500$

0.11

UV 15

0.11
0.09
0.05

UV 14

U V 14

$416-43109 \quad 0.05$

U V 14

U V 13

$0-42533$

0.00

U V 11

$416-42860$

$7377-49805$

$888-43079$

$704-42860$

0.05

0.91

0.11

U V 11

UV 11

U V $11 \quad-0.97$
0.86 U V 71

$7377-51361$

0.09 U V 16
1.16

1.18

0.82

0.45

0.43

0.34

0.31

$-0.50$

$-0.79$

$-0.40$

$-0.11$

$-0.79$

$-0.65$

$-0.08$

$-0.78$

$-1.19$

$-0.35$

$-0.62$

$-0.36$

$-1.08$

$-0.31$

$-0.59$

$-0.59$

$-0.41$

$-0.28$

$-0.99$

$-0.97$

$-0.36$

$-0.26$

$-0.41$

$-0.35$

$-0.26$

$-0.71$

$-0.58$

$-1.00$

$-0.26$

$-0.24$

$-1.30$

$-0.56$

$-0.58$

$-0.82$

$-0.77$

$-0.68$

$-0.49$

$-0.32$

$-0.31$

$-1.42$

$-1.69$

$-1.33$

$-0.20$

$\mathrm{R}(\mathrm{N}) \quad \mathrm{KOC}$

Best

$\log g f \lambda$

$\log$

$\mathrm{g} A / \lambda$

7.03

6.38

6.97

6.06

$\begin{array}{lll}0.80 & 4.14 & 6.61 \\ 0.45 & 3.79 & 6.26\end{array}$

$\begin{array}{lll}0.56 & 3.90 & 6.36\end{array}$

$\begin{array}{rrr}0.562 & 3.32 & 5.78 \\ -0.02 & 3.90 & 6.36\end{array}$

$\begin{array}{lll}0.49 & 3.83 & 6.29\end{array}$

$\begin{array}{lll}0.44 & 3.78 & 6.24\end{array}$

$\begin{array}{lll}0.34 & 3.68 & 6.14\end{array}$

$\begin{array}{rrr}0.31 & 3.65 & 6.11 \\ -0.50 & 2.85 & 5.27\end{array}$

$\begin{array}{lll}-0.79 & 2.56 \quad 4.98\end{array}$

$\begin{array}{lll}-0.40 & 2.95 & 5.36\end{array}$

$\begin{array}{lll}-0.11 & 3.24 & 5.65\end{array}$

$\begin{array}{lll}-0.79 & 2.57 & 4.97\end{array}$

$\begin{array}{lll}-0.61 & 2.75 \quad 5.15\end{array}$

$\begin{array}{lll}-0.65 & 2.71 & 5.11\end{array}$

$-0.08$ 
TABLE 3.-Oscillator Strengths for Ultraviolet Lines of Fe I-Continued

\begin{tabular}{|c|c|c|c|c|c|c|c|c|c|c|c|c|}
\hline $\begin{array}{l}\text { Wave- } \\
\text { length } \\
\text { ¿ }\end{array}$ & $\begin{array}{c}\text { Energy Levels } \\
\mathrm{K}\end{array}$ & $\begin{array}{l}\text { Low } \\
\text { e.p. } \\
\text { Volts }\end{array}$ & $\begin{array}{l}\text { Mult. } \\
\text { No. }\end{array}$ & Arc & Spk & $\mathrm{CB}$ & $\begin{array}{l}\mathrm{HC} \\
-\log g f\end{array}$ & $\mathrm{KOC}$ & MS & Best & $\log g f \lambda$ & $\begin{array}{l}\log \\
\mathrm{gA} / \lambda\end{array}$ \\
\hline $\begin{array}{l}2373.625 \\
2374.519 \\
2381.836 \\
2389.973 \\
2408.045\end{array}$ & $\begin{array}{r}416-42533 \\
978-43079 \\
888-42860 \\
704-42533 \\
7728-49243\end{array}$ & $\begin{array}{l}0.05 \\
0.12 \\
0.11 \\
0.09 \\
0.96\end{array}$ & $\begin{array}{ll}\text { UV V } & 11 \\
\text { UV } & 11 \\
\text { UV V } & 11 \\
\text { UV V } & 11 \\
\text { UV } & 68\end{array}$ & $\begin{array}{l}-0.63 \\
-1.06 \\
-0.88 \\
-0.86 \\
-1.84\end{array}$ & & & & & & $\begin{array}{l}-0.63 \\
-1.06 \\
-0.88 \\
-0.86 \\
-1.84\end{array}$ & $\begin{array}{l}2.75 \\
2.32 \\
2.50 \\
2.52 \\
1.54\end{array}$ & $\begin{array}{l}5.07 \\
4.64 \\
4.81 \\
4.83 \\
3.84\end{array}$ \\
\hline $\begin{array}{l}2411.558 \\
2429.810 \\
2433.056 \\
2438.183 \\
2439.630\end{array}$ & $\begin{array}{r}6928-48383 \\
8155-49298 \\
8155-49243 \\
6928-47930 \\
11976-52954\end{array}$ & $\begin{array}{l}0.86 \\
1.01 \\
1.01 \\
0.86 \\
1.48\end{array}$ & $\begin{array}{ll}\text { U V } & 68 \\
\text { UV } & 68 \\
\text { UV } & 62\end{array}$ & $\begin{array}{l}-1.11 \\
-1.40 \\
-0.78 \\
-0.52 \\
-0.86\end{array}$ & & & & & & $\begin{array}{l}-1.11 \\
-1.40 \\
-0.78 \\
-0.52 \\
-0.86\end{array}$ & $\begin{array}{l}2.27 \\
1.99 \\
2.61 \\
2.87 \\
2.53\end{array}$ & $\begin{array}{l}4.57 \\
4.27 \\
4.89 \\
5.14 \\
4.80\end{array}$ \\
\hline $\begin{array}{l}2439.743 \\
2440.106 \\
2442.567 \\
2443.873 \\
2445.213\end{array}$ & $\begin{array}{r}19390-60366 \\
19788-60758 \\
19621-60549 \\
6928-47835 \\
6928-47812\end{array}$ & $\begin{array}{l}2.40 \\
2.45 \\
2.43 \\
0.86 \\
0.86\end{array}$ & $\begin{array}{l}\text { U V } 157 \\
\text { U V } 157 \\
\text { UV } 157 \\
\text { UV } 63 \\
\text { U V } 63\end{array}$ & $\begin{array}{r}0.89 \\
0.48 \\
0.56 \\
-0.37 \\
-1.09\end{array}$ & & $\begin{array}{r}0.50 \\
0.37 \\
0.66 \\
-0.33\end{array}$ & & & & $\begin{array}{r}0.70 \\
0.42 \\
0.61 \\
-0.35 \\
-1.09\end{array}$ & $\begin{array}{l}4.09 \\
3.81 \\
4.00 \\
3.04 \\
2.30\end{array}$ & $\begin{array}{l}6.36 \\
6.08 \\
6.27 \\
5.31 \\
4.57\end{array}$ \\
\hline $\begin{array}{l}2447.711 \\
2450.439 \\
2451.384 \\
2453.477 \\
2453.568\end{array}$ & $\begin{array}{r}0-40842 \\
12561-53358 \\
12969-53749 \\
7377-48123 \\
19621-60366\end{array}$ & $\begin{array}{l}0.00 \\
1.56 \\
1.61 \\
0.91 \\
2.43\end{array}$ & $\begin{array}{l}\text { U V } 62 \\
\text { U V } 157\end{array}$ & $\begin{array}{l}-1.08 \\
-0.84 \\
-1.03 \\
-0.21 \\
-0.40\end{array}$ & & $\begin{array}{l}-1.04 \\
-0.24\end{array}$ & & & & $\begin{array}{l}-1.06 \\
-0.84 \\
-1.03 \\
-0.22 \\
-0.40\end{array}$ & $\begin{array}{l}2.33 \\
2.55 \\
2.36 \\
3.17 \\
2.99\end{array}$ & $\begin{array}{l}4.60 \\
4.82 \\
4.63 \\
5.43 \\
5.25\end{array}$ \\
\hline $\begin{array}{l}2457.598 \\
2458.564 \\
2462.182 \\
2462.648 \\
2463.728\end{array}$ & $\begin{array}{r}6928-47606 \\
6928-47590 \\
416-41018 \\
0-40594 \\
7728-48305\end{array}$ & $\begin{array}{l}0.86 \\
0.86 \\
0.05 \\
0.00 \\
0.96\end{array}$ & $\begin{array}{lr}\text { UV } & 62 \\
\text { UV } & 59 \\
\text { UV } & 9 \\
\text { UV } & 9 \\
\text { UV } & 65\end{array}$ & $\begin{array}{r}0.30 \\
-1.38 \\
-0.72 \\
-0.26 \\
-0.53\end{array}$ & & & & & & $\begin{array}{r}0.30 \\
-1.38 \\
-0.72 \\
-0.26 \\
-0.53\end{array}$ & $\begin{array}{l}3.69 \\
2.01 \\
2.67 \\
3.13 \\
2.86\end{array}$ & $\begin{array}{l}5.95 \\
4.27 \\
4.93 \\
5.39 \\
5.12\end{array}$ \\
\hline $\begin{array}{l}2465.150 \\
2467.733 \\
2468.880 \\
2470.961 \\
2472.343\end{array}$ & $\begin{array}{l}7377-47930 \\
7728-48239 \\
6928-47420 \\
7377-47834 \\
6928-47363\end{array}$ & $\begin{array}{l}0.91 \\
0.96 \\
0.86 \\
0.91 \\
0.86\end{array}$ & $\begin{array}{ll}U V & 62 \\
U V & 62 \\
U V & 59 \\
U V & 63 \\
U V & 59\end{array}$ & $\begin{array}{r}0.19 \\
-0.48 \\
0.20 \\
-0.70 \\
0.00\end{array}$ & & 更 & & & & $\begin{array}{r}0.19 \\
-0.48 \\
0.20 \\
-0.70 \\
0.03\end{array}$ & $\begin{array}{l}3.58 \\
2.91 \\
3.59 \\
2.69 \\
3.42\end{array}$ & $\begin{array}{l}5.84 \\
5.17 \\
5.85 \\
4.95 \\
5.67\end{array}$ \\
\hline $\begin{array}{l}2472.896 \\
2473.156 \\
2474.815 \\
2476.654 \\
2476.861\end{array}$ & $\begin{array}{r}416-40842 \\
0-40422 \\
7728-48123 \\
7986-48351 \\
8155-48516\end{array}$ & $\begin{array}{l}0.05 \\
0.00 \\
0.96 \\
0.99 \\
1.01\end{array}$ & $\begin{array}{lr}\text { UV } & 9 \\
\text { UV } & 8 \\
\text { UV } & 62 \\
\text { UV } & 62 \\
\text { UV } & 65\end{array}$ & $\begin{array}{r}-0.06 \\
-0.98 \\
0.23 \\
-0.38 \\
-1.37\end{array}$ & & $\begin{array}{l}0.14 \\
0.33\end{array}$ & & & & $\begin{array}{r}0.04 \\
-0.98 \\
0.28 \\
-0.38 \\
-1.37\end{array}$ & $\begin{array}{l}3.43 \\
2.41 \\
3.67 \\
3.01 \\
2.02\end{array}$ & $\begin{array}{l}5.68 \\
4.66 \\
5.92 \\
5.26 \\
4.27\end{array}$ \\
\hline $\begin{array}{l}2479.481 \\
2479.777 \\
2483.272 \\
2483.531 \\
2484.186\end{array}$ & $\begin{array}{r}7986-48305 \\
704-41018 \\
0-40257 \\
7986-48239 \\
888-41131\end{array}$ & $\begin{array}{l}0.99 \\
0.09 \\
0.00 \\
0.99 \\
0.11\end{array}$ & $\begin{array}{lr}\text { UV } & 65 \\
\text { UV } & 9 \\
\text { UV } & 9 \\
\text { UV } & 62 \\
\text { UV } & 9\end{array}$ & $\begin{array}{r}-0.27 \\
0.00 \\
-0.18 \\
-0.02\end{array}$ & & $\begin{array}{r}-0.15 \\
0.14 \\
0.49 \\
0.11\end{array}$ & & & & $\begin{array}{r}-0.21 \\
0.07 \\
0.49 \\
-0.18 \\
0.05\end{array}$ & $\begin{array}{l}3.18 \\
3.46 \\
3.89 \\
3.22 \\
3.45\end{array}$ & $\begin{array}{l}5.43 \\
5.71 \\
6.13 \\
5.46 \\
5.69\end{array}$ \\
\hline $\begin{array}{l}2484.530 \\
2485.989 \\
2486.372 \\
2486.690 \\
2487.064\end{array}$ & $\begin{array}{r}11976-52213 \\
7377-47590 \\
0-40207 \\
7728-47930 \\
8155-48351\end{array}$ & $\begin{array}{l}1.48 \\
0.91 \\
0.00 \\
0.96 \\
1.01\end{array}$ & $\begin{array}{lr}\text { UV } & 59 \\
\text { UV } & 8 \\
U V & 62 \\
\text { UV } & 62\end{array}$ & $\begin{array}{l}-0.92 \\
-0.74 \\
-0.15 \\
-0.02\end{array}$ & & $\begin{array}{l}-1.00 \\
-0.18 \\
-0.03\end{array}$ & & & & $\begin{array}{l}-0.92 \\
-0.74 \\
-1.00 \\
-0.16 \\
-0.02\end{array}$ & $\begin{array}{l}2.48 \\
2.66 \\
2.40 \\
3.24 \\
3.38\end{array}$ & $\begin{array}{l}4.72 \\
4.90 \\
4.64 \\
5.48 \\
5.62\end{array}$ \\
\hline $\begin{array}{l}2487.370 \\
2488.144 \\
2488.942 \\
2489.751 \\
2489.917\end{array}$ & $\begin{array}{r}704-40895 \\
416-40594 \\
20641-60807 \\
978-41131 \\
8155-48305\end{array}$ & $\begin{array}{l}0.09 \\
0.05 \\
2.56 \\
0.12 \\
1.01\end{array}$ & $\begin{array}{lr}\text { U V } & 10 \\
\text { U V } & 9 \\
\text { U V } 164 \\
\text { UV } & 9 \\
\text { U V } & 65\end{array}$ & $\begin{array}{r}-1.28 \\
0.06 \\
0.07 \\
-0.25\end{array}$ & & $\begin{array}{r}-1.33 \\
0.51 \\
0.41\end{array}$ & & & & $\begin{array}{r}-1.30 \\
0.51 \\
0.06 \\
0.24 \\
-0.25\end{array}$ & $\begin{array}{l}2.10 \\
3.91 \\
3.46 \\
3.64 \\
3.15\end{array}$ & $\begin{array}{l}4.34 \\
6.15 \\
5.70 \\
5.88 \\
5.39\end{array}$ \\
\hline $\begin{array}{l}2490.645 \\
2491.156 \\
2491.983 \\
2492.640 \\
2493.998\end{array}$ & $\begin{array}{r}704-40842 \\
888-41018 \\
20641-60758 \\
7728-47834 \\
7728-47812\end{array}$ & $\begin{array}{l}0.09 \\
0.11 \\
2.56 \\
0.96 \\
0.96\end{array}$ & $\begin{array}{lr}\text { U V } & 9 \\
\text { U V } & 9 \\
\text { U V } 163 \\
\text { UV } & 63 \\
\text { U V } & 63\end{array}$ & $\begin{array}{r}0.16 \\
-0.02 \\
-1.06 \\
-0.55\end{array}$ & & $\begin{array}{l}0.38 \\
0.29\end{array}$ & & & & $\begin{array}{r}0.38 \\
0.22 \\
-0.02 \\
-1.06 \\
-0.55\end{array}$ & $\begin{array}{l}3.78 \\
3.62 \\
3.38 \\
2.34 \\
2.85\end{array}$ & $\begin{array}{l}6.01 \\
5.85 \\
5.61 \\
4.57 \\
5.08\end{array}$ \\
\hline $\begin{array}{l}2494.252 \\
2495.869 \\
2496.534 \\
2501.133 \\
2501.695\end{array}$ & $\begin{array}{r}6928-47008 \\
6928-46982 \\
7377-47420 \\
0-39970 \\
6928-46889\end{array}$ & $\begin{array}{l}0.86 \\
0.86 \\
0.91 \\
0.00 \\
0.86\end{array}$ & $\begin{array}{lr}\text { U V } & 57 \\
\text { U V } & 57 \\
\text { U V } & 59 \\
\text { U V } & 7 \\
\text { U V } & 56\end{array}$ & $\begin{array}{r}-1.06 \\
-0.55 \\
0.17 \\
-0.06 \\
-0.66\end{array}$ & $\begin{array}{l}-0.11 \\
-0.57\end{array}$ & $\begin{array}{r}0.08 \\
-0.02 \\
-0.58\end{array}$ & & -0.58 & & $\begin{array}{r}-1.06 \\
-0.55 \\
0.12 \\
-0.06 \\
-0.60\end{array}$ & $\begin{array}{l}2.34 \\
2.85 \\
3.52 \\
3.34 \\
2.80\end{array}$ & $\begin{array}{l}4.57 \\
5.08 \\
5.75 \\
5.57 \\
5.03\end{array}$ \\
\hline
\end{tabular}


TABLE 3.-Oscillator Strengths for Ultraviolet Lines of Fe I-Continued

Wave-

Le-

ength

.

Energy Levels Low K Volts

Mult.

Arc Spk

CB HC R(N) KOC MS

Best

Log

No.

$-\log g f$

2503.491

2504.101

2504.635

2505.004

2506.569

$20875-60807$

2.59

$20641-60564 \quad 2.56$

$17727-57641 \quad 2.20$

$20641-60549 \quad 2.56$

$20875-60758 \quad 2.59$

U V164 - -0.15

-1.20
-2.01

U V163 -0.54

2507.899

2508.751

2508.948

2509.390

2510.836

2512.266

2512.361

2515.848

2516.249

2516.572

2517.658

2518.103

2518.824

2519.630

2521.920

2522.488

2522.850

2524.294

2527.436

2528.910

2529.136

2529.837

2530.694

2531.510

2532.876

2535.128

2535.609

2537.460

2539.358

2539.575

2540.973

2542.101

2543.920

2544.706

2545.980

2549.614

2550.812

2552.604

2552.832

2554.518

2555.648

2556.304

2556.862

2557.268

2560.558

2561.262

2561.856

2562.224

2563.820

2564.561

2567.860

2568.862

2569.595

2569.742

2571.570
$7728-47590 \quad 0.96$

$7986-47834$

$7986-47831$

$17727-57565$

416-40231

$6928-46721$

$416-40207$

$12561-52297$

$7377-47107$

$7728-47453$

$7986-47693$

$704-40405$

$20875-60564$

$8155-4783$

$7377-47017$

$7377-47008$

$0-39626$

$888-40491$

$416-39970$

$20641-60172$

$704-40231$

888-40405

704-40207

$20875-60365$

$7728-47197$

$7986-47420$

$978-40405$

$11976-51374$

$7377-46745$

$7728-47093$

$888-40231$

21039-60365

$20875-60172$

$20641-59927$

$704-39970$

$416-39626$

$7986-47177$

$888-40052$

$7728-46889$

22947-62081

$8155-47272$

$12561-51668$

$6928-46027$

$11976-51069$

$8155-47197$

$7986-47017$

$8155-47177$

$8155-47172$

$7728-46721$

$8155-47136$

$17927-56859$

$7986-46902$

$6928-45833$

$7986-46889$

$12561-51436$
0.96 U V 59

$\begin{array}{lll}0.99 & \text { UV } 63 & -0.98\end{array}$

$0.99 \quad$ U V 59 -2.01

2.20

0.05

UV 7

$-0.16$

UV $55-0.68$

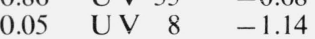

1.56 UV104 -1.15

0.91 UV $57 \quad-1.48$

$0.96 \quad$ U V $61 \quad-0.96$

$0.91 \quad$ UV $58 \quad-0.84$

0.91 U V 57

$-0.75$

UV 7

0.11 UV 7

0.05 UV 7

2.56 U V 162

$-0.17$

0.15

UV 7 -0.06

$\begin{array}{lll}0.09 & \text { UV } 8 & -1.28\end{array}$

2.59 UV162 -0.20

0.96 UV $56-1.20$

$0.99 \quad$ U V 60

0.12 UV 7

1.48 U V 102

0.91 UV 55

$-1.20$

$-0.18$

$-0.49$

$-0.78$

UV 7

2.61 UV162

2.59 U V 162

$2.56 U$ V 162

0.09 U V 7

0.9

0.91

0.76

$-1.28$

UV 55

U V 8

$-1.58$

-1.07
-0.93

U V 58

U V 102

U V 53

U V 101

$-1.58$

$-0.50$

$-1.02$

$-1.40$

U V $56-1.11$

$0.99 \quad$ U V 58

$-1.39$

U V 55

$-1.20$

$-0.88$

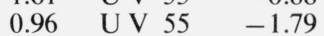

$1.01 \quad$ U V $58 \quad-1.19$

U V130 -0.72

U V $54-1.06$

U V $52-1.03$

U V $55 \quad-0.99$

U V103
$0.04 \quad 0.01$

$-0.07-0.05$

0.04

$-1.12$

0.27

$-1.10$

$-1.16$

$-0.98$

$-1.13$

$-0.12$

$-0.18$

$-0.27$

$\begin{array}{llll}\text { U V } 59 & -0.31 & -0.01 & -0.24\end{array}$

$0.56-0.44$

$\begin{array}{rr}-0.23 & -0.20 \\ -0.37 & 0.18\end{array}$

$-0.25$

0.18

$0.11-0.23$

$-0.71$

0.96 UV 56

$-0.18-0.06$

-0.42
-0.75

-0.13
-1.52
0.32

$-1.32$

$-1.62$

$-0.33$

$-0.45$

$-0.77$

$-2.00$

$-0.22-0.02$

$\begin{array}{ll}1.07 & 0.87 \\ 0.99 & 0.86\end{array}$

0.50
$-0.11 \quad-0.05$

2.05

1.94

$-0.39-0.18$

$-1.72$

1.01 U V 55

$-1.80$

$\begin{array}{lll}-0.15 & 3.25 & 5.48\end{array}$

$-1.20$

$\begin{array}{lll}-2.01 & 1.39 & 3.62\end{array}$

$\begin{array}{lll}-0.54 & 2.86 & 5.09\end{array}$

$\begin{array}{lll}-0.54 & 2.86 & 5.09 \\ -0.24 & 3.16 & 5.39\end{array}$

$\begin{array}{lll}-0.01 & 3.39 & 5.62\end{array}$

$\begin{array}{lll}-1.05 & 2.35 & 4.58\end{array}$

$\begin{array}{lll}-2.01 & 1.39 & 3.62\end{array}$

$\begin{array}{lll}-1.48 & 1.92 & 4.15\end{array}$

$\begin{array}{lll}0.04 & 3.44 \quad 5.66\end{array}$

$\begin{array}{lll}-0.68 & 2.72 & 4.94\end{array}$

$\begin{array}{lll}-1.14 & 2.26 & 4.48\end{array}$

$\begin{array}{lll}-1.15 & 2.25 & 4.47\end{array}$

$\begin{array}{lll}-0.98 & 2.42 & 4.64\end{array}$

$\begin{array}{lll}-1.04 & 2.36 & 4.58\end{array}$

$\begin{array}{lll}-0.12 & 3.28 & 5.50\end{array}$

$\begin{array}{lll}-0.08 & 3.32 & 5.54\end{array}$

$\begin{array}{lll}-0.49 & 2.91 & 5.13\end{array}$

$\begin{array}{lll}-0.21 & 3.19 & 5.41\end{array}$

$\begin{array}{lll}-0.84 & 2.56 & 4.78\end{array}$

$\begin{array}{lll}-0.75 & 2.65 & 4.87\end{array}$

$\begin{array}{lll}0.50 & 3.90 & 6.12\end{array}$

$\begin{array}{lll}-0.20 & 3.20 & 5.42\end{array}$

$\begin{array}{lll}0.23 & 3.63 & 5.85\end{array}$

$\begin{array}{rrr}0.23 & 3.63 & 5.85 \\ -0.25 & 3.15 & 5.37\end{array}$

$\begin{array}{lll}-0.08 & 3.32 & 5.54\end{array}$

$\begin{array}{lll}-0.59 & 2.81 & 5.02\end{array}$

$\begin{array}{lll}-1.30 & 2.10 \quad 4.31\end{array}$

$-1.26$

2.14

4.35

$\begin{array}{ll}-0.19 & 3.21\end{array}$

$\begin{array}{ll}-0.45 & 2.95\end{array}$

$-0.77 \quad 2.63$

5.42

$\begin{array}{ll}.63 & 4.84\end{array}$

$\begin{array}{lll}-0.12 & 3.29 & 5.49\end{array}$

$\begin{array}{lll}0.63 & 4.04 \quad 6.24\end{array}$

$\begin{array}{lll}-0.08 & 3.33 & 5.53\end{array}$

$\begin{array}{lll}-0.28 & 3.13 & 5.32\end{array}$

$\begin{array}{ll}-1.28 & 2.13 \\ -1.65 & -4.32\end{array}$

$\begin{array}{lll}-1.65 & 1.76 & 3.95\end{array}$

$\begin{array}{lll}-1.12 & 2.29 & 4.48\end{array}$

$\begin{array}{lll}-0.93 & 2.48 & 4.67\end{array}$

$-1.69$

1.72 
Table 3.-Oscillator Strengths for Ultraviolet Lines of Fe I-Continued

length Energy Levels e.p. K Volts

Mult

CB HC R(N) KOC MS Best

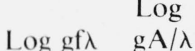

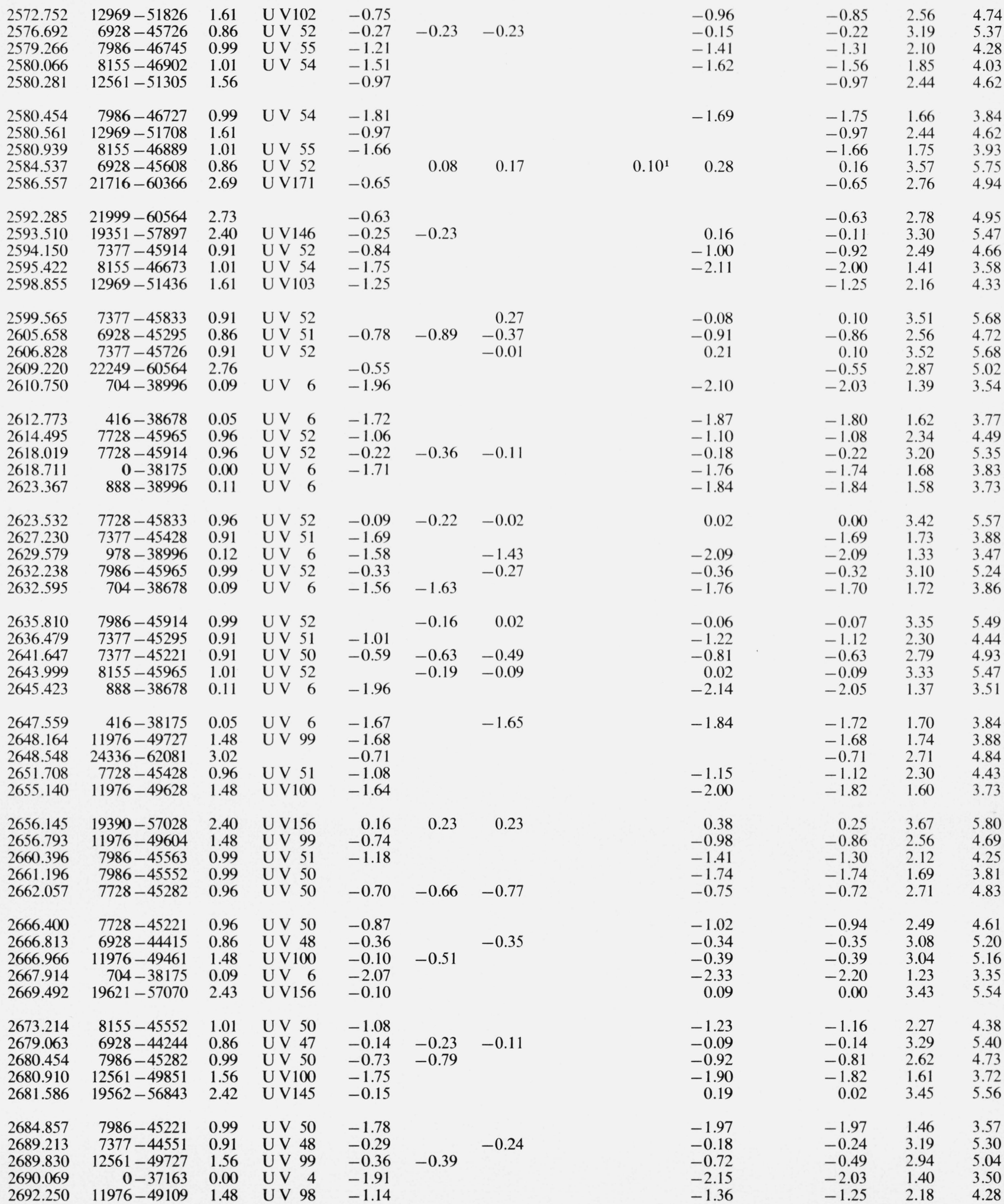


TABLE 3.-Oscillator Strengths for Ultraviolet Lines of $\mathrm{Fe} \mathrm{I}-\mathrm{Continued}$

Wave- Low

length Energy Levels Low $\AA \quad K \quad$ Volts

Mult.

CB HC R(N) KOC MS Best

No. $-\log g f$

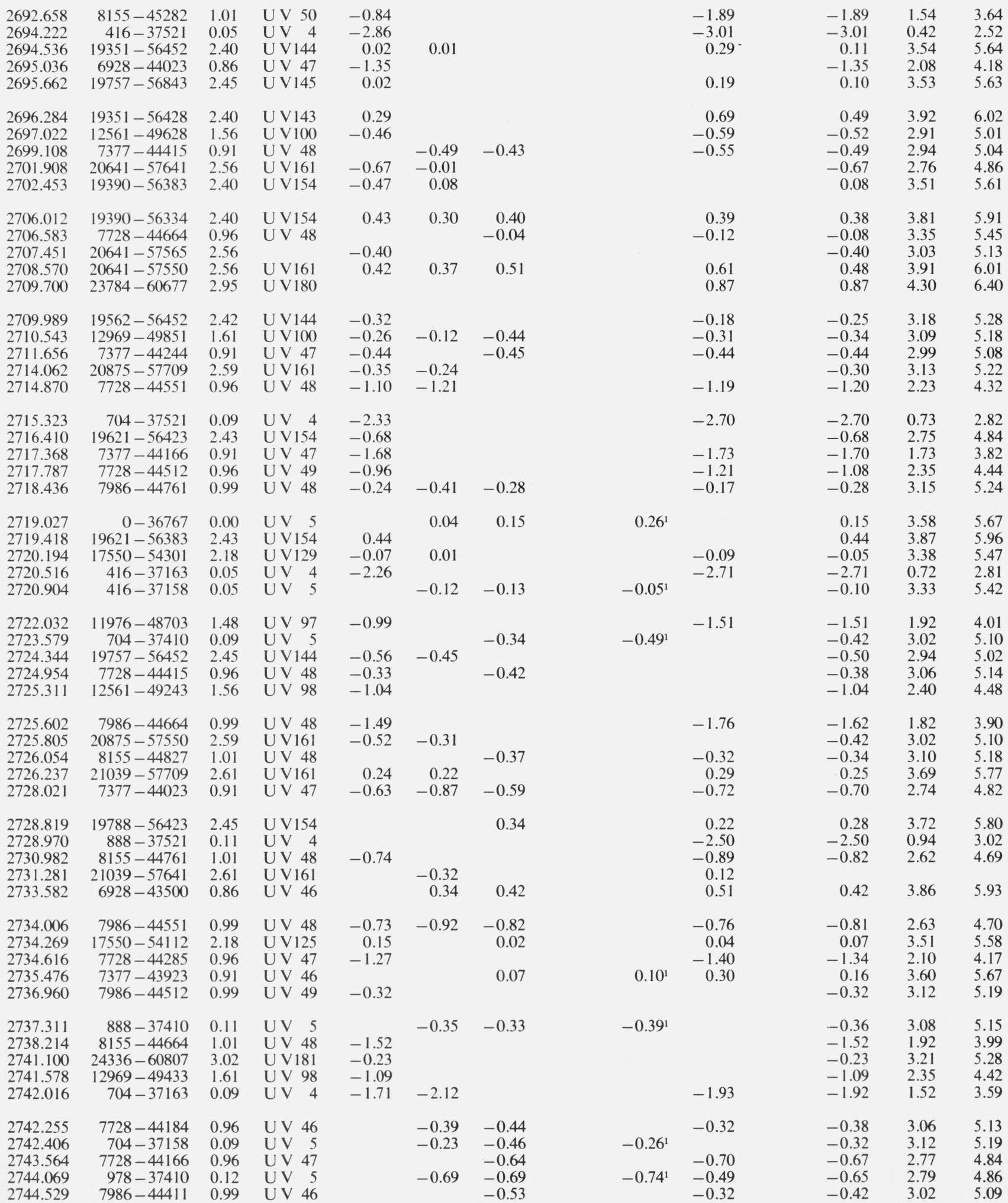


TABLE 3.-Oscillator Strengths for Ultraviolet Lines of Fe I-Continued

Wave- Low

length Energy Levels Low A $\quad \mathrm{K} \quad$ Volts

Mult. No.

Arc Spk $\quad \mathrm{CB} \quad \mathrm{HC}$

2746.982

2747.556

2749.688

2750.142

2750.708

2750.872

2751.808

2753.687

2754.033

2754.427

2755.184

2756.268

2756.330

2757.317

2759.814

2761.480

2761.78

2762.028

2762.773

2763.108

2764.32

2765.700

2766.910

2767.523

2768.432

2769.298

2769.672

2770.695

2772.075

2772.320

2772.511

2772.860

2773.907

2774.150

2774.730

2778.221

2780.526

2780.700

2781.837

2782.055

2784.017

2784.346

2786.180

2787.120

2787.933

2788.106

2789.477

2789.803

2790.762

2791.787

2792.397

2794.157

2794.703

2795.006

2795.541

2796.872

2797.776

2802.285

2803.168

2803.613

$$
\begin{array}{r}
6928-43321 \\
17727-54112 \\
8155-44512 \\
416-36767 \\
17927-54271
\end{array}
$$

$12969-49298$

$8155-44459$

$7986-44285$

$7728-44023$

$19621-55906$

416-36686

$888-37158$

$8155-44411$

$8155-44378$

$19552-55754$

$7986-44184$

$7728-43923$

$17927-54112$

$7986-44166$

$17727-53892$

$11976-48123$

$8155-44285$

$7377-43500$

$17550-53661$

$19390-55490$

$6928-43023$

$17727-53808$

$6928-42992$

$17550-53610$

$17727-53785$

$24119-60172$

$19390-55430$

$17727-53763$

$8155-44184$

$6928-42912$

$11976-47930$

$20641-56593$

$7986-43923$

$17727-53661$

$20875-56783$

$19621-55526$

$17927-53808$

19621

$11976-47835 \quad 1.48$

$6928-42784 \quad 0.86$

$17550-53389 \quad 2.18$

$21716-57550 \quad 2.69$

$17927-53749 \quad 2.22$

$19621-55430 \quad 2.43$

$12561-48362$

$17550-53329$

$7728-43500$

$0-35768$

$7377-43138$

$12561-48305$

$7377-43109$

$26406-62081$

$416-36079$

$19788-55446$

2.99

2.40

1.01

0.86
1.48

2.56

0.99
2.20

1.56

0.96

0.00

1.56

0.91

3.27

0.05
$17550-53892$

UV $5-2.00$
0.86 U V 45

2.20 U V125

1.01 UV 49

0.05 UV 5

U V 125

$-0.21$

$-0.11$

0.13

$-0.38 \quad-0.40$

$-0.35^{1}$

$-1.00$

$-0.34$

U V128

0.18

$-1.34$

U V $46 \quad-0.55$

UV $47 \quad-0.58$

U V $47-0.87$

U V153

UV $4-1.06$

U V $46 \quad-0.35$

U V 47

$-0.69$

$\begin{array}{ll}-0.84 & -0.87\end{array}$

$-0.62-0.40$

$-0.84-1.01$

U V $140 \quad-0.87$

0.99 U V 46

U V 46

U V 125

U V 47

0.87

$-0.15$

$-0.16$

$-0.16$

$-0.46$

$-0.64$

0.14

$-1.80$

$-0.76$

$-0.82$

$-0.16$

U V 92

U V 47

U V 46

U V126

$-0.54$

$-1.15-1.02$
-

U V151

U V 44

U V 123

UV 45

0.26

$-1.31$

$-0.73$

$\begin{array}{lll}-0.24 & -0.44 & -0.22\end{array}$

$-0.35$

$-0.43$

U V179

$-0.57$

U V151 -1.40

U V127 - -1.50

U V $46-0.93$

U V 44

U V $92-1.74$

U V $160 \quad-0.48$

U V 46

U V126

$-0.74$

$-0.76-0.81$

U V160 -0.95

UV152 - -0.71

UV123 -1.20

UV151 -1.20

U V 93

U V 44

U V 125

U V 170

$-0.43$

-0.43
-0.06

$\begin{array}{ll}\text { U V151 } & -1.58 \\ -0.25\end{array}$

U V $95-1.03$

2.18 UV124 -1.32

U V 46

UV $3-2.29$

U V $44-1.10$

U V $96-1.51$

U V 45

$-1.01$

UV $3 \quad-2.74$

$-0.64 \quad-0.54$

$0.54 \quad 0.35$

0.01

$0.08 \quad 0.02$

$-1.11$

$-1.22$

$-2.05$

U V151 -0.43

$-1.99$

$-0.83$

$-0.39$

$-0.60$

0.26

$-1.47$

$-0.07$

$-0.39$

$-1.05$

$\mathrm{R}(\mathrm{N}) \quad \mathrm{KOC} \quad \mathrm{MS}$

Best $\log g f \lambda \quad g A / \lambda$

$\begin{array}{lll}0.13 & 3.57 & 5.64\end{array}$

$\begin{array}{lll}-0.18 & 3.26 & 5.33\end{array}$

$\begin{array}{lll}-1.33 & 2.11 \quad 4.18\end{array}$

$\begin{array}{lll}-0.38 & 3.06 & 5.13\end{array}$

$\begin{array}{lll}-0.34 & 3.10 \quad 5.17\end{array}$

$\begin{array}{lll}0.16 & 3.60 & 5.67\end{array}$

$\begin{array}{lll}-1.34 & 2.10 & 4.17\end{array}$

$\begin{array}{lll}-0.59 & 2.85 & 4.91\end{array}$

$\begin{array}{lll}-0.63 & 2.81 & 4.87\end{array}$

$\begin{array}{lll}-1.02 & 2.42 \quad 4.48\end{array}$

$\begin{array}{lll}-0.53 & 2.91 & 4.97\end{array}$

$\begin{array}{lll}-1.04 & 2.40 \quad 4.46\end{array}$

$\begin{array}{lll}-0.60 & 2.84 & 4.90\end{array}$

$\begin{array}{lll}-0.30 & 3.14 & 5.20\end{array}$

$\begin{array}{lll}-0.83 & 2.61 & 4.67\end{array}$

$\begin{array}{lll}-0.87 & 2.57 & 4.63\end{array}$

$\begin{array}{lll}-0.20 & 3.24 & 5.30\end{array}$

$\begin{array}{lll}-0.15 & 3.29 & 5.35\end{array}$

$\begin{array}{lll}-0.30 & 3.14 & 5.20\end{array}$

$\begin{array}{lll}-0.70 & 2.74 & 4.80\end{array}$

$\begin{array}{lll}-0.01 & 3.43 & 5.49\end{array}$

$-1.90 \quad 1.54$

$-0.94 \quad 2.50$

$\begin{array}{lll}0.26 & 3.70 & 5.76\end{array}$

$\begin{array}{lll}-1.39 & 2.05 & 4.11\end{array}$

$\begin{array}{lll}-0.32 & 3.12 & 5.18\end{array}$

$\begin{array}{lll}-0.35 & 3.09 & 5.15\end{array}$

$-0.43 \quad 3.01 \quad 5.07$

$\begin{array}{lll}-0.46 & 2.98 & 5.04\end{array}$

$\begin{array}{lll}-1.40 & 2.04 & 4.09\end{array}$

$\begin{array}{lll}-1.50 & 1.94 & 3.99\end{array}$

$\begin{array}{lll}-0.94 & 2.50 \quad 4.55\end{array}$

$\begin{array}{lll}-0.34 & 3.10 & 5.15\end{array}$

$\begin{array}{lll}-1.74 & 1.70 & 3.75\end{array}$ 
TABLE 3.-Oscillator Strengths for Ultraviolet Lines of Fe I-Continued

Wave-

Low

Low
ength Energy Levels e.p.

A K Volts

2804.521

2805.808

2806.072

2806.985

2807.246

2808.328

2810.834

2811.160

2812.042

2813.288

2815.017

2815.506

2817.505

2819.286

2820.804

2821.630

2823.277

2824.700

2825.557

2825.689

2825.995

2827.893

2828.809

2832.436

2833.401

2834.177

2834.414

2834.755

2835.457

2835.951

2836.315

2838.120

2840.423

2840.938

2843.631

2843.978

2845.596

2845.714

2846.831

2848.715

2851.798

2852.952

2853.685

2853.774

2857.996

2858.897

2861.996

2862.495

2863.431

2863.864

2866.626

2867.311

2867.563

2867.880

2868.213

2868.454

2869.308

2869.833

2871.730

$21999-57641$

$18378-54005 \quad 2.28$

$0-35612 \quad 0.00$

$7728-43326 \quad 0.96$

$21999-57565 \quad 2.73$

$12561-48123 \quad 1.56$

$21999-57550 \quad 2.73$

$7377-42912 \quad 0.91$

$18378-53892$

$12969-48476$

$7728-43210$

$22249-57709$

$18378-53808 \quad 2.28$

$7728-43138$
0.96

$22249-57641 \quad 2.76$

$0-35379$

0.00

$704-36079$

416-35768

$7986-43326$

$7728-43023$

0.05

$18378-53661$

$12561-47834$

$12969-48239$

$20641-55907$

$0-35257$

2.28

$12561-47812$

$22650-57897$

$7986-43210$

416-35612

$17727-52916$

$7377-42533$

$7986-43138$

$7728-42860$

$11976-47107$

$11976-47093$

$7986-43079$

$8155-43210$

$11976-47017$

$11976-47008$

$20875-55906$

0.00

2.20

0.99

$888-35856$

$17927-52858$

$8155-43079$

11976-46889

2.28

$704-35612$

$12969-47834 \quad 1.6$

$12561-47420$

$19757-54612 \quad 2.45$

$416-35257 \quad 0.05$

$19913-54748 \quad 2.47$

$\begin{array}{ll}19788-54600 & 2.45\end{array}$
Mult.
No.

Arc

$7377-43023 \quad 0.91 \quad$ U V 44

U V170

UV 92

$-0.39$

U V $139-1.03$
I

UV 45

$-2.52$

U V 2

-2.52
-1.55

$-1.21$

U V $92-1.90$

2.28

U V170

$-0.85$

1.61 UV 95

$-1.14$

$-1.06$

0.2

0.16

$-1.12$

$-0.21$

$\begin{array}{ll}416-35856 & 0.05\end{array}$

U V170

$-2.83$

U V 2

$-2.83$

UV134

U V 44

$-1.73$

$-0.95$

$-0.15 \quad 0.27$

$-0.30^{1}$

$-0.20-0.26$

$-2.01$

U V 45

U V

0.99 U V 45

$-2.92$

$-2.29$

U V 44

$\begin{array}{rrr}-2.29 & -0.81 & -1.01\end{array}$

$\begin{array}{rr}-0.81 & -1.01 \\ 0.05\end{array}$

$-0.91$

$-1.97$

$\begin{array}{ll}\text { UV 92 } & -1.95 \\ \text { UV159 } & -0.74 \\ \text { UV } 2 & -2.04\end{array}$

$\begin{array}{ll}\text { UV 92 } & -1.95 \\ \text { UV159 } & -0.74 \\ \text { UV } 2 & -2.04\end{array}$

U V 2

$-1.11$

2.81 UV175 -0.78

0.99 U V 44

0.05 UV 2

U V 123

$-2.02$

$-1.11$

$-1.99$

U V 43

0.99 U V 44

0.96 U V 43

1.48 U V 88

U V 87

U V 43

U V 44

U V 89

UV 88

$18378-53358$

U V159

$-1.97$

$-0.61$

\begin{tabular}{ll}
$-0.47 \quad-0.13$ \\
\hline
\end{tabular}

$$
-0.42
$$$$
-1.95
$$

$\begin{array}{rr}-1.22 & -1.20\end{array}$

$-0.53-0.54$

$\begin{array}{rr}0.13 & -0.01 \\ -0.80 & -0.61\end{array}$

$-0.07$

$\begin{array}{ll}-0.95 & -0.47\end{array}$

$-2.20-2.5$

U V

$\begin{array}{ll} & -1.78 \\ \text { U V } 43 & -1.33\end{array}$

UV 87

$12969-47831 \quad 1.61$

1.56

UV 2

$-0.41$

$-0.56 \quad-0.25$

$$
\begin{array}{r}
-1.22 \\
0.17
\end{array}
$$

$\begin{array}{lll}-1.63 & -1.84 & -1.73\end{array}$

U V $43 \quad-0.97 \quad-0.99 \quad-1.05$

$18378-53230 \quad 2.28$
UV 93

$-0.86$

$-1.62$

U V142

U V135

U V 2

U V142

U V149

$-0.72$

$-0.45$ $\begin{array}{lllll}-1.48 & -1.57 & -1.47 & -1.68^{1} & -1.62\end{array}$

HC R(N) KOC MS Best

$\log$

$\begin{array}{lll}-0.32 & 3.13 & 5.16\end{array}$

$\begin{array}{lll}-0.39 & 3.06 & 5.09\end{array}$

$\begin{array}{lll}-1.61 & 1.84 & 3.87\end{array}$

$\begin{array}{lll}-1.03 & 2.42 & 4.45\end{array}$

$\begin{array}{lll}-0.21 & 3.24 & 5.27\end{array}$

$\begin{array}{lll}-2.68 & 0.77 & 2.80\end{array}$

$\begin{array}{lll}-1.43 & 2.02 \quad 4.05\end{array}$

$\begin{array}{lll}-1.21 & 2.24 & 4.27\end{array}$

$\begin{array}{lll}-1.90 & 1.55 & 3.58\end{array}$

$\begin{array}{lll}-0.85 & 2.60 & 4.63\end{array}$

$\begin{array}{lll}0.23 & 3.68 & 5.71\end{array}$

$\begin{array}{lll}-1.14 & 2.31 \quad 4.34\end{array}$

$\begin{array}{lll}-1.08 & 2.37 & 4.40\end{array}$

$\begin{array}{lll}-1.12 & 2.33 & 4.35\end{array}$

$\begin{array}{lll}-0.21 & 3.24 & 5.26\end{array}$

$\begin{array}{lll}-2.83 & 0.62 & 2.64\end{array}$

$\begin{array}{lll}-1.73 & 1.72 & 3.74\end{array}$

$\begin{array}{lll}-0.22 & 3.23 & 5.25\end{array}$

$\begin{array}{lll}-0.95 & 2.50 & 4.52\end{array}$

$\begin{array}{lll}-0.23 & 3.22 & 5.24\end{array}$

$\begin{array}{lll}-1.96 & 1.49 & 3.51\end{array}$

$\begin{array}{lll}-2.92 & 0.53 & 2.55\end{array}$

$\begin{array}{lll}-2.29 & 1.16 & 3.18\end{array}$

$\begin{array}{lll}-0.91 & 2.54 & 4.56\end{array}$

$\begin{array}{lll}0.00 & 3.45 & 5.47\end{array}$

$\begin{array}{lll}-0.91 & 2.54 & 4.56\end{array}$

$\begin{array}{lll}-1.97 & 1.48 & 3.50\end{array}$

$\begin{array}{lll}-1.95 & 1.50 & 3.52\end{array}$

$\begin{array}{lll}-0.74 & 2.71 & 4.73\end{array}$

$\begin{array}{lll}-2.02 & 1.43 & 3.45\end{array}$

$\begin{array}{lll}-1.16 & 2.29 & 4.31\end{array}$

$\begin{array}{lll}-0.78 & 2.67 & 4.69\end{array}$

$\begin{array}{lll}-0.44 & 3.01 & 5.02\end{array}$

$\begin{array}{lll}-1.98 & 1.47 & 3.48\end{array}$

$\begin{array}{lll}-1.11 & 2.34 & 4.35\end{array}$

$\begin{array}{lll}-0.54 & 2.91 \quad 4.92\end{array}$

$\begin{array}{lll}0.02 & 3.47 & 5.48\end{array}$

$\begin{array}{lll}-0.70 & 2.75 & 4.76\end{array}$

$\begin{array}{lll}0.09 & 3.54 & 5.55\end{array}$

$\begin{array}{lll}-1.27 & 2.18 & 4.19\end{array}$

$\begin{array}{lll}-1.18 & 2.27 & 4.28\end{array}$

$\begin{array}{lll}-0.02 & 3.44 & 5.44\end{array}$

$\begin{array}{lll}-1.99 & 1.47 & 3.47\end{array}$

$\begin{array}{lll}-1.50 & 1.96 & 3.96\end{array}$

$\begin{array}{lll}-0.28 & 3.18 \quad 5.18\end{array}$

$\begin{array}{lll}-0.47 & 2.99 & 4.99\end{array}$ 
TABLE 3.-Oscillator Strengths for Ultraviolet Lines of Fe I-Continued

Wave- Low

length Energy Levels Low

A $\quad \mathrm{K}$ Volts

2872.335

2873.655

2874.173

2874.890

2875.303

2877.302

2878.762

2878.962

2879.461

2880.581

2886.317

2887.360

2887.806

2887.961

2889.890

2889.991

2890.414

2890.868

2891.410

2891.705

2891.904

2892.479

2893.763

2893.882

2894.506

2895.036

2897.635

2898.867

2899.416

2901.382

2901.910

2905.570

2907.518

2908.864

2909.313

2910.930

2912.159

2914.306

2918.023

2918.354

2919.838

2920.290

2920.691

2922.383

2922.620

2923.288

2923.851

2924.002

2925.359

2925.901

2928.105

2929.008

2929.118

2929.620

2930.590

2931.420

2931.803

2934.370

2936.120

2936.905

\begin{tabular}{|c|c|c|c|c|c|}
\hline $7728-42533$ & 0.96 & U V 43 & -0.86 & & -0.78 \\
\hline $20641-55430$ & 2.56 & U V158 & -0.83 & & \\
\hline $0-34782$ & 0.00 & U V 2 & & -1.59 & -1.43 \\
\hline $19351-54125$ & 2.40 & U V142 & -0.63 & -0.23 & \\
\hline $11976-46745$ & 1.48 & UV 86 & -0.84 & & -0.56 \\
\hline $11976-46721$ & 1.48 & U V 86 & -0.36 & -0.45 & -0.26 \\
\hline $22838-57565$ & 2.83 & & -0.53 & & \\
\hline $12969-47693$ & 1.61 & UV 90 & -1.67 & & \\
\hline $19552-54271$ & 2.42 & U V136 & -1.38 & & \\
\hline $8155-42860$ & 1.01 & U V 43 & -1.93 & & \\
\hline $12561-47197$ & 1.56 & UV 87 & -1.17 & & \\
\hline $19390-54014$ & 2.40 & U V150 & -1.65 & & \\
\hline $21716-56334$ & 2.69 & U V167 & -0.19 & & -0.29 \\
\hline $19621-54237$ & 2.43 & U V149 & -1.14 & & \\
\hline $19390-53983$ & 2.40 & U V149 & -0.90 & & \\
\hline $20020-54612$ & 2.48 & U V142 & -0.80 & & \\
\hline $12969-47556$ & 1.61 & & -2.24 & & \\
\hline $26225-60807$ & 3.25 & U V184 & -1.02 & & \\
\hline $12561-47136$ & 1.56 & UV 89 & -1.99 & & \\
\hline $20875-55446$ & 2.59 & U V158 & & -0.34 & \\
\hline $19788-54357$ & 2.45 & & -0.94 & -0.38 & \\
\hline $19562-54125$ & 2.42 & U V142 & -0.63 & & \\
\hline $7986-42533$ & 0.99 & U V 43 & -1.80 & & \\
\hline $12561-47107$ & 1.56 & UV 88 & -1.11 & & \\
\hline $18378-52916$ & 2.28 & U V134 & 0.19 & 0.28 & 0.45 \\
\hline $12561-47093$ & 1.56 & UV 87 & -0.45 & -0.42 & -0.31 \\
\hline $19788-54289$ & 2.45 & & & -0.73 & \\
\hline $17727-52213$ & 2.20 & & -1.37 & & \\
\hline $18378-52858$ & 2.28 & U V133 & 0.00 & 0.12 & 0.15 \\
\hline $12561-47017$ & 1.56 & UV 89 & -0.72 & -0 & \\
\hline $19351-53801$ & 2.40 & U V142 & -0.15 & & -0.10 \\
\hline $26351-60758$ & 3.27 & 32 & -0.66 & -0.38 & \\
\hline $21999-56383$ & 2.73 & U V167 & -0.07 & 0.13 & 0.12 \\
\hline $19757-54125$ & 2.45 & U V142 & -0.57 & & \\
\hline $19621-53983$ & 2.43 & U V149 & -1.40 & & \\
\hline $22249-56593$ & 2.76 & U V168 & -0.98 & & \\
\hline $0-34329$ & 0.00 & & & -1.35 & -1.17 \\
\hline $12969-47272$ & 1.61 & UV 89 & -0.76 & -0.79 & \\
\hline $26106-60366$ & 3.24 & U V182 & 0.59 & 0.64 & 0.75 \\
\hline $19552-53808$ & 2.42 & U V134 & -0.41 & -0 & \\
\hline $19562-53801$ & 2.42 & U V142 & -0.69 & -0 & \\
\hline $20038-54271$ & 2.4 & & -1.50 & & \\
\hline $12969-47197$ & 1.61 & UV 87 & -0.47 & -0.59 & -0.19 \\
\hline $12969-47177$ & 1.61 & UV 86 & -1.51 & & \\
\hline $17550-51756$ & 2.18 & U V122 & -0.84 & -0.89 & \\
\hline $26351-60549$ & 3.27 & L & 0.25 & 0. & 0.75 \\
\hline $21716-55907$ & 2.6 & & 0.10 & 0. & 0.41 \\
\hline $21716-55906$ & 2.6 & & 0.30 & 0.2 & \\
\hline $22249-56423$ & 2.76 & & -0.10 & 0. & \\
\hline $12969-47136$ & 1.61 & UV 89 & -0.79 & -0 & \\
\hline $17550-51692$ & 2.1 & U V121 & -1.07 & & \\
\hline $416-34547$ & 0.0 & & & -1 . & \\
\hline $26628-60758$ & 3.3 & U V182 & & 0.17 & \\
\hline $12969-47093$ & 1.61 & UV 87 & -1.23 & & -1.14 \\
\hline $20020-54132$ & 2.48 & UV141 & -1.95 & & \\
\hline $19788-53892$ & 2.4 & & -1.15 & & \\
\hline $21999-56098$ & 2.7 & & -0.94 & & \\
\hline $17550-51619$ & 2.1 & & -1.38 & & \\
\hline $12969-47017$ & 1.61 & UV 89 & -1.21 & & \\
\hline $0-34040$ & 0.00 & UV 1 & & -0.56 & -0.59 \\
\hline
\end{tabular}

Mult.

CB HC R(N) KOC MS Best $\log \operatorname{gf} \lambda \quad \mathrm{gA} / \lambda$

$\log$

4.59

$\begin{array}{rrr}-0.86 & 2.60 & 4.59 \\ -0.83 & 2.63 & 4.62\end{array}$

$\begin{array}{lllll}-1.65^{1} & -1.54 & -1.55 & 1.91 & 3.90\end{array}$

$\begin{array}{llll}-0.88 & -0.76 & 2.70 & 4.69\end{array}$

$-0.22$

$-0.32$

3.14

5.13

2.93

$\begin{array}{lll}-1.67 & 1.79 & 3.78\end{array}$

$\begin{array}{lll}-1.38 & 2.08 & 4.07\end{array}$

$-1.96$

$-1.94$

1.52

3.51

$-1.37$

$-1.27$

2.19

4.17

$\begin{array}{lll}-1.65 & 1.81 & 3.79\end{array}$

$\begin{array}{lll}-0.24 & 3.22 & 5.20\end{array}$

$\begin{array}{lll}-1.14 & 2.32 & 4.30\end{array}$

$\begin{array}{lll}-0.90 & 2.56 & 4.54\end{array}$

$\begin{array}{lll}-0.80 & 2.66 & 4.64\end{array}$

$\begin{array}{lll}-2.24 & 1.22 & 3.20\end{array}$

$\begin{array}{lll}-1.02 & 2.44 & 4.42\end{array}$

$\begin{array}{lll}-1.99 & 1.47 & 3.45\end{array}$

$\begin{array}{lll}-0.34 & 3.12 & 5.10\end{array}$

$\begin{array}{lll}-0.94 & 2.52 & 4.50\end{array}$

$-1.85$

$\begin{array}{lll}-0.63 & 2.83 & 4.81\end{array}$

$\begin{array}{lll}-1.82 & 1.64 & 3.62\end{array}$

$\begin{array}{lll}-1.06 & 2.40 \quad 4.38\end{array}$

-1.02
0.45

0.34

$3.80 \quad 5.78$

$-0.35$

3.11

5.09

$-0.73$

2.73

$\begin{array}{lll}-1.37 & 2.09 & 4.07\end{array}$

$\begin{array}{rrrr}0.06 & 0.08 & 3.54 & 5.52 \\ -0.47 & -0.65 & 2.81 & 4.79\end{array}$

$-0.01$

$-0.09$

3.37

5.35

$\begin{array}{lll}-0.52 & 2.94 & 4.91\end{array}$

$\begin{array}{lll}0.06 & 3.52 & 5.49\end{array}$

$\begin{array}{lll}-0.57 & 2.89 & 4.86\end{array}$

$\begin{array}{lll}-1.40 \quad 2.06 & 4.03\end{array}$

$\begin{array}{lll}-0.98 & 2.48 & 4.45\end{array}$

$\begin{array}{llllll}-1.22^{2} & -1.38 & -1.11 & -1.24 & 2.22 & 4.19\end{array}$

$-1.28$

$\begin{array}{lll}0.66 & 4.13 \quad 6.09\end{array}$

$\begin{array}{lll}-0.42 & 3.05 & 5.01\end{array}$

$\begin{array}{lll}-0.61 & 2.86 & 4.82\end{array}$

$\begin{array}{lll}-1.50 & 1.97 \quad 3.93\end{array}$

$\begin{array}{llll}-0.44 & -0.42 & 3.05 & 5.01 \\ -1.61 & -1.56 & 1.91 & 3.87\end{array}$

$\begin{array}{lll}-0.86 & 2.61 & 4.57\end{array}$

$\begin{array}{lll}0.47 & 3.94 & 5.90\end{array}$

$\begin{array}{lll}0.27 & 3.74 & 5.70\end{array}$

$\begin{array}{lll}0.29 & 3.76 & 5.72\end{array}$

$\begin{array}{lll}-0.03 & 3.44 & 5.40\end{array}$

$\begin{array}{lll}-0.84 & 2.63 & 4.59\end{array}$

$-0.94$

$-1.07$

2.40

4.35

$\begin{array}{lll}-1.12^{2} & -1.21 & -1.03\end{array}$

$-1.12$

2.35

3.64

4.30

5.59

$\begin{array}{lll}-1.24 & 2.23 & 4.18\end{array}$

$\begin{array}{lll}-1.95 & 1.52 & 3.47\end{array}$

$\begin{array}{lll}-1.15 & 2.32 & 4.27\end{array}$

$\begin{array}{lll}-0.94 & 2.53 & 4.48\end{array}$

$\begin{array}{lll}-1.38 & 2.09 & 4.04\end{array}$

$\begin{array}{lll}-1.21 & 2.26 & 4.21\end{array}$

$-0.66^{3}$

$-0.60 \quad 2.87$

4.82 
TABLE 3.-Oscillator Strengths for Ultraviolet Lines of Fe I-Continued

Wave-

length Energy Levels Low

A K Volts

$2937.806 \quad 17727-51756 \quad 2.20$

2939.072

$17927-51942 \quad 2.22$

2940.586

2941.344

2941.770

2945.050

2.83

$704-34692-0.09$

$19562-53546 \quad 2.42$

$19788-53734 \quad 2.45$

2945.870

2946.095

2947.116

2947.363

$26628-60564$

$12969-46902$

$26628-60549$

$18378-52297$

2947.877

2948.433

2948.733

2949.688

2950.240

416-34329

$21999-55906$

$17727-51630$

$17727-51619$

$17550-51436$

$19788-53661$

2951.356

2953.486

2953.941

2954.654

2956.710

2956.860

2957.366

2958.462

2959.682

2959.993

2960.299

2960.666

2961.700

2962.110

2963.710

2964.196

2965.256

2965.81

2966.260

2966.900

$22249-56098$

$704-34547$

$18378-52213$

$17550-51361$

$21716-55526$

$888-34692$

$21999-55791$

$22650-56428$

$21716-55490$

3.30

1.61

3.30

2.28

0.05

2.73

2.20

2.20

2.18

2.45

2.76

0.09

2.28

2.18

20038-53808

$23784-57550$

$17550-51305$

$11976-45726$

$23111-56843$

2.69

0.11

2.73

2.81

2.69

2.48

2.95

2.18

1.48

$20875-54600$

$978-34692$

$19621-53329$

$\begin{array}{rr}19621-53329 & 2.43 \\ 17927-51630 & 2.22\end{array}$

$0-33695$

2.59

0.12

2968.481

2969.361

2969.476

2970.106

2972.277

$19552-53230 \quad 2.42$ $888-34556 \quad 0.11$ $6928-40594 \quad 0.86$ $888-34547 \quad 0.11$

$17727-51361 \quad 2.20$

2973.134

2973.237

2974.780

2975.655

2976.126

2976.550

2976.922

2978.060

2980.532

2981.446

2981.852

2982.234

2983.571

2984.785

2986.457

$416-34040$

$22846-56452$

$20641-54237$

$18378-51969$

$11976-45563$

$22846-56428$

$19788-53358$

$22249-55791$

$416-33947$

$17550-51077$

24119-57641

$0-33507$

$6928-40422$

$888-34363$

$19621-53094 \quad 2.43$

2986.653

2987.292

2988.473

2988.942

2990.393

2.83

2.56

Mult. No.

U V 122

UV118

UV173

U V 1

U V 141

\begin{tabular}{lrr} 
& -0.45 & -0.52 \\
& -1.39 & \\
U V182 & -2.25 & \\
U V131 & 0.24 & \\
\hline & -0.42 & -0.32
\end{tabular}

U V 1

U V 166

U V118

U V 117

U V120

0.25

$-0.45 \quad-0.55$

$-0.99$

$-1.54$

0.08

$\begin{array}{lr}0.23 & -0.26\end{array}$

$\mathrm{HC}$
$-\mathrm{Log} g f$

$\mathrm{R}(\mathrm{N})$ KOC MS Best

$\begin{array}{ll}\log g f \lambda & \operatorname{gA} / \lambda\end{array}$

$-1.27$

U V 166

0.12

U V132

U V 118

$-0.13$

0.36

$-0.62-0.65$

U V165 -0.72

$\begin{array}{rr}10 & \\ 317 & -1.39\end{array}$

U V172

$-0.01$

0.62

$0.74 \quad 0.61$

148

$-0.39$

$-0.28$

$-0.63$

U V119 - -2.29

$-2.02$

U V173 -1.24

10

$-1.18$

U V 147

104
10

$-0.49$

$-0.80$

$-0.96-1.15$

$-0.92$

$-0.29$

U V135 -0.67

$11-1.65$

30
10

104

$-0.50$

$-1.61-1.82$

$\begin{array}{ll}-0.74 & -0.77\end{array}$

$-0.73-0.89$

$-0.50$

10

10

335

$-0.95$

$$
-0.46 \quad-0.67
$$

$-0.39-0.40$

146

$-0.55$

$-0.20$

$-0.12-0.47$

$-1.74$

$-1.05$

$-1.39$

$317-0.04$

11

$104-0.07$

$460-0.98$

9

29

11

$-0.89$

$-2.12$

$\begin{array}{ll}0.16 & -0.27\end{array}$

$-1.00 \quad-1.04$

$0.06-0.45$

$-0.38 \quad-0.39$
-0.61

$$
-2.35
$$

$-0.53^{3}$

$-0.48$

-

$-0.34$

3.13

$-0.78$

$\begin{array}{llll}-1.32^{2} & -1.28 & -1.25 & -0.40\end{array}$

2.69

3.07

2.16

$-0.81$

2.66

$-0.48$

$-1.39$

$-2.25$

2.99

2.08

1.22
3.71

$-0.37$

3.10

$-0.42$

$-0.43$

0.25

$-0.99$

$-1.54$

$-0.39$

$-0.28$

$\begin{array}{rr} & -1.27 \\ & 0.24 \\ -0.50 \quad-0.61\end{array}$

$\begin{array}{rr} & -1.27 \\ & 0.24 \\ -0.50 \quad-0.61\end{array}$

$-0.63^{3}$

$-0.23$

3.04

3.72

2.48

1.93

3.19

2.20

3.71

2.86

3.24

2.47

$-0.72$

2.75

$-0.72^{2} \quad-0.75 \quad-0.69$

$-0.77$

$-1.39$

2.70

2.08

0.66

3.46
4.13

$-0.34$

$-0.63$ 
TABLE 3.-Oscillator Strengths for Ultraviolet Lines of Fe I-Continued

Wave-

length Low $\begin{array}{lll}\text { i } & \mathrm{K} & \text { e.p. } \\ & & \end{array}$

\begin{tabular}{|c|c|c|}
\hline 2994.428 & $416-33802$ & 0.05 \\
\hline 2994.503 & $978-34363$ & 0.12 \\
\hline 2995.838 & $24339-57709$ & 3.02 \\
\hline 2996.386 & $19552-52916$ & 2.42 \\
\hline 2999.512 & $6928-40257$ & 0.86 \\
\hline 3000.453 & $11976-45295$ & 1.48 \\
\hline 3000.949 & $704-34017$ & 0.09 \\
\hline 3001.663 & $24336-57641$ & 3.02 \\
\hline 3003.032 & $7728-41018$ & 0.96 \\
\hline 3004.119 & $19621-52899$ & 2.43 \\
\hline 3004.620 & $12561-45833$ & 1.56 \\
\hline 3005.302 & $19390-52655$ & 2.40 \\
\hline 3006.598 & $20641-53892$ & 2.56 \\
\hline 3007.147 & $11976-45221$ & 1.48 \\
\hline 3007.283 & $704-33947$ & 0.09 \\
\hline 3008.140 & $888-34122$ & 0.11 \\
\hline 3009.094 & $19390-52613$ & 2.40 \\
\hline 3009.571 & $7377-40594$ & 0.91 \\
\hline 3011.482 & $22249-55446$ & 2.76 \\
\hline 3011.883 & $20038-53230$ & 2.48 \\
\hline 3014.175 & $7728-40895$ & 0.96 \\
\hline 3015.913 & $19621-52769$ & 2.43 \\
\hline 3016.186 & $7986-41131$ & 0.99 \\
\hline 3017.629 & $888-34017$ & 0.11 \\
\hline 3018.134 & $19390-52514$ & 2.40 \\
\hline 3018.985 & $7728-40842$ & 0.96 \\
\hline 3019.290 & $19788-52899$ & 2.45 \\
\hline 3020.492 & $704-33802$ & 0.09 \\
\hline 3020.640 & $0-33096$ & 0.00 \\
\hline 3021.074 & $416-33507$ & 0.05 \\
\hline 3024.034 & $888-33947$ & 0.11 \\
\hline 3025.283 & $7377-40422$ & 0.91 \\
\hline 3025.638 & $19390-52431$ & 2.40 \\
\hline 3025.844 & $978-34017$ & 0.12 \\
\hline 3026.464 & $7986-41018$ & 0.99 \\
\hline 3029.235 & $12561-45563$ & 1.56 \\
\hline 3030.149 & $19621-52613$ & 2.43 \\
\hline 3030.605 & $18378-51365$ & 2.28 \\
\hline 3031.213 & $19788-52769$ & 2.45 \\
\hline 3031.638 & $8155-41131$ & 1.01 \\
\hline 3033.101 & $19552-52512$ & 2.42 \\
\hline 3034.510 & $12969-45914$ & 1.61 \\
\hline 3037.390 & $888-33802$ & 0.11 \\
\hline 3037.781 & $7986-40895$ & 0.99 \\
\hline 3039.322 & $19621-52514$ & 2.43 \\
\hline 3040.428 & $7377-40257$ & 0.91 \\
\hline 3041.639 & $12561-45428$ & 1.56 \\
\hline 3041.740 & $7728-40594$ & 0.96 \\
\hline 3042.022 & $8155-41018$ & 1.01 \\
\hline 3042.667 & $7986-40842$ & 0.99 \\
\hline 3045.077 & $7377-40207$ & 0.91 \\
\hline 3045.594 & $19788-52613$ & 2.45 \\
\hline 3046.930 & $19621-52431$ & 2.43 \\
\hline 3047.050 & $23784-56593$ & 2.95 \\
\hline 3047.606 & $704-33507$ & 0.09 \\
\hline 3053.065 & $19552-52297$ & 2.42 \\
\hline 3053.440 & $8155-40895$ & 1.01 \\
\hline 3055.264 & $12561-45282$ & 1.56 \\
\hline 3056.250 & $21039-53749$ & 2.61 \\
\hline 3057.447 & $6928-39626$ & 0.86 \\
\hline
\end{tabular}

Mult. Arc Spk $\mathrm{CB} \quad \mathrm{HC} \quad \mathrm{R}(\mathrm{N})$ KOC MS Best No. $-\log g f$

$\begin{array}{rrrr}9 & & -0.34 & -0.42 \\ 11 & -0.23 & & \\ 460 & -1.30 & & \\ 148 & -0.09 & 0.01 & -0.40 \\ 30 & & 0.10 & -0.15 \\ & & & \\ 56 & -0.23 & -0.33 & -0.39 \\ 9 & & -0.35 & -0.43 \\ 506 & -0.85 & & \\ 30 & & -0.50 & -0.61 \\ 199 & -0.56 & & \\ & & & \\ 57 & -1.87 & & \\ 199 & -0.38 & -0.18 & \\ & -1.20 & & \\ 55 & -0.17 & -0.32 & -0.55 \\ 11 & & -1.26 & -1.48 \\ & & & \\ 9 & & -0.50 & -0.50 \\ 198 & -0.03 & 0.04 & \\ 30 & & -0.09 & -0.21 \\ 316 & 0.50 & 0.54 & 0.43\end{array}$

31
198
30
9
199
30
199
9
9
9

11

29
198
9
30

30

$\begin{array}{rrrr}56 & -1.05 & -1.02 & \\ 198 & 0.71 & 0.78 & 0.87 \\ 145 & -1.37 & & \\ 198 & 0.57 & 0.72 & 0.88 \\ 30 & & -0.47 & -0.31 \\ 146 & -0.73 & -0.65 & \end{array}$

$\begin{array}{ll}57 & -0.73 \\ 9 & -1.30\end{array}$

$31-1.56$

$0.32-0.50$

$199 \quad-0.77 \quad-0.38$

$\begin{array}{llll}30 & -0.60 & -0.64 & -0.46 \\ 56 & & -0.26 & -0.21\end{array}$

$\begin{array}{lll}30 & -0.50 & -0.57 \\ 30 & -0.69 & -0.82\end{array}$

$\begin{array}{llll}30 & -0.21 & -0.53 & -0.34\end{array}$

$\begin{array}{rrrr}29 & -1.12 & -1.29 & \\ 198 & -0.88 & -0.51 & \\ 198 & -0.76 & -0.52 & \\ 457 & -0.32 & -0.17 & \\ 9 & & -0.31 & -0.40 \\ & & & \\ 146 & 0.05 & 0.06 & \\ 31 & -1.67 & & \\ 55 & -0.13 & -0.33 & -0.29 \\ & -0.72 & & \\ 28 & & 0.22 & 0.05\end{array}$

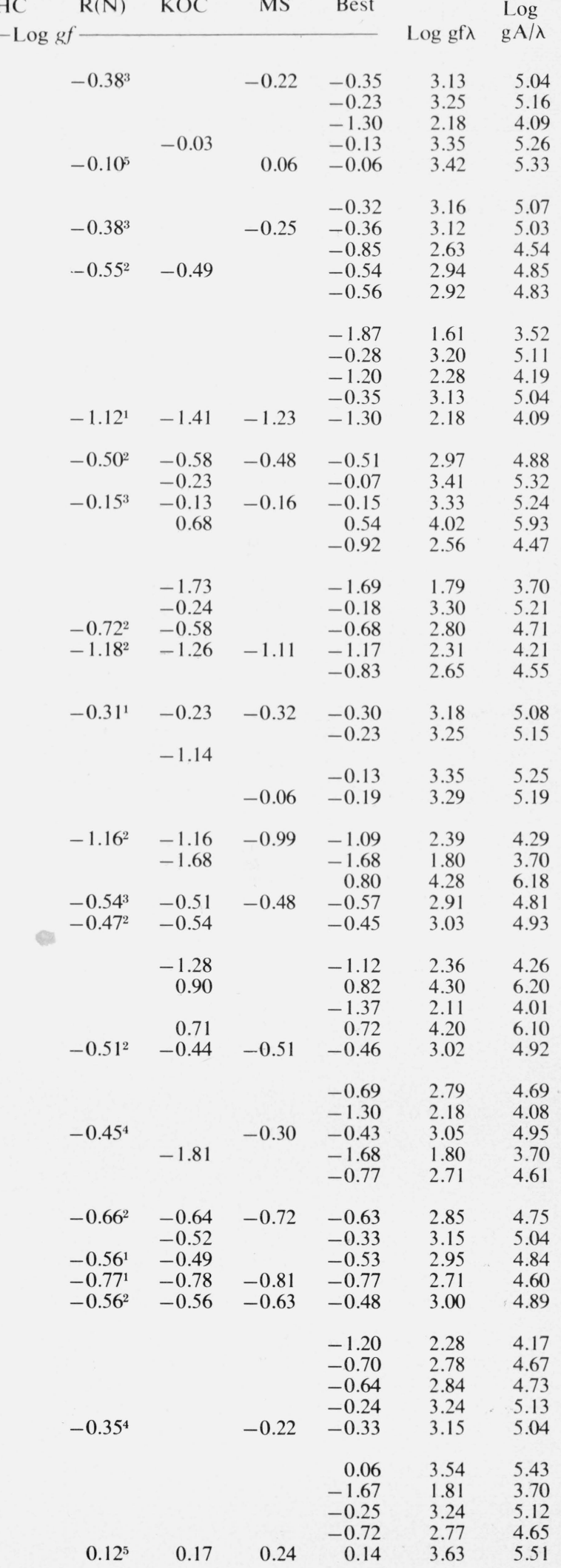


TABLE 3.-Oscillator Strengths for Ultraviolet Lines of Fe I-Continued

Wave-

$\AA \quad$ K $\quad$ Volts

3057.802

3059.087

3060.545

3060.985

3062.872

3063.149

3063.933

3066.483

3067.246

3067.952

3068.175

3073.244

3073.982

3074.157

3075.721

3078.014

3078.436

3081.278

3081.832

3083.152

3083.743

3087.420

3090.209

3091.579

3092.778

3093.806

3094.870

3095.270

3096.044

3098.192

3098.963

3099.897

3099.970

3100.305

3100.667

3100.838

3101.004

3102.644

3103.760

3107.978

3109.050

3111.686

3112.079

3113.592

3114.054

3115.656

3115.862

3116.250

3116.634

3116.984

3117.640

3119.496

3120.220

3120.436

3121.760

3123.353

3123.545

3124.099

3125.653

3128.901
$7728-40422 \quad 0.96$ 416-33096 0.05 $\begin{array}{ll}24119-56783 & 2.99\end{array}$

$12561-45221 \quad 1.56$

$23784-56423 \quad 2.95$

$17550-50187 \quad 2.18$

$19552-52181 \quad 2.42$

$21999-54600 \quad 2.73$

$7377-39970 \quad 0.91$

$21716-54301 \quad 2.69$

$12969-45552 \quad 1.61$

$24575-57104 \quad 3.05$

$21716-54237 \quad 2.69$

$24339-56859 \quad 3.02$

$7728-40231 \quad 0.96$

$7728-40207 \quad 0.96$

$20038-52512 \quad 2.48$

$24339-56783 \quad 3.02$

$11976-44415 \quad 1.48$

$19788-52213 \quad 2.45$

$7986-40405 \quad 0.99$

$26140-58520 \quad 3.24$

$22249-54600 \quad 2.76$

$8155-40491 \quad 1.01$

$7728-40052 \quad 0.96$

$12969-45282 \quad 1.61$

$21999-54301 \quad 2.73$

$21716-54014 \quad 2.69$

$21999-54289 \quad 2.73$

$21716-53983 \quad 2.69$

$17927-50189 \quad 2.22$

$8155-40405 \quad 1.01$

$7377-39626 \quad 0.91$

$\begin{array}{ll}7986-40231 & 0.99\end{array}$

$7728-39970 \quad 0.96$

$19390-51630 \quad 2.40$

$21999-54237 \quad 2.73$

7986-40207 0.99

$19552-51762 \quad 2.42$

$\begin{array}{ll}21716-53882 & 2.69\end{array}$

$19913-52067 \quad 2.47$

$20641-52769 \quad 2.56$

23784-55907 2.95

$22249-54357 \quad 2.76$

$12561-44664 \quad 1.56$

$24772-56859 \quad 3.07$

$24339-56423 \quad 3.02$

$19757-51837 \quad 2.45$

$8155-40231 \quad 1.01$

$26140-58213 \quad 3.24$

$7986-40052 \quad 0.99$

$19621-51668 \quad 2.43$

$22249-54289 \quad 2.76$

$19788-51826 \quad 2.45$

$17927-49951 \quad 2.22$

$19562-51570 \quad 2.42$

$21999-54005 \quad 2.73$

$20020-52020 \quad 2.48$

$7986-39970 \quad 0.99$

$12561-44512 \quad 1.56$ $\begin{array}{lllllll}\text { Arc } & \text { Spk } & \text { CB } & \text { HC } & \text { R(N) KOC } & \text { MS Best }\end{array}$

Mult. No.

$-\log g f$

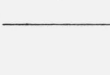

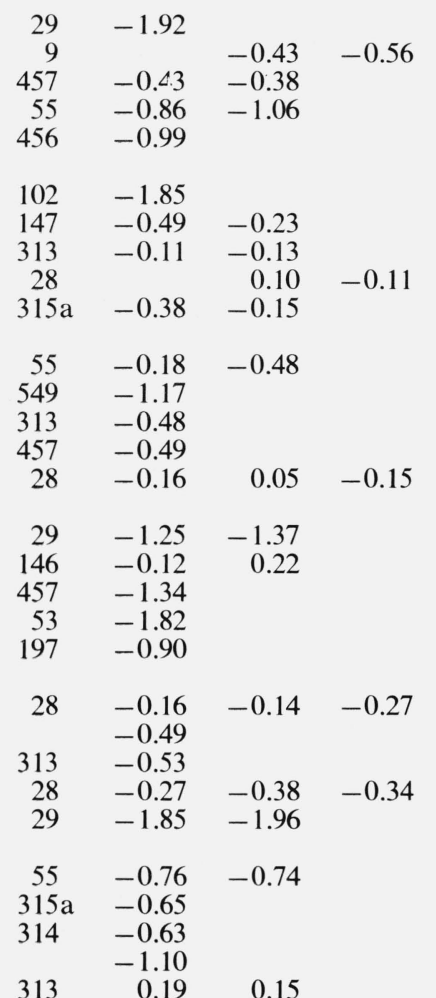

$\begin{array}{rrr}-0.44^{4} & & -0.37 \\ & -1.23 & \\ & & \\ & & \\ & & \\ -0.05^{5} & 0.30\end{array}$

$-1.92$

$\log \operatorname{gf} \lambda$

$\log$

$\mathrm{gA} / \lambda$

$$
\begin{array}{rrrr}
102 & -1.37 & & \\
28 & 0.04 & -0.02 & 0.10
\end{array}
$$

$\begin{array}{lll}-0.26 & 3.23\end{array}$

\begin{tabular}{|c|c|c|}
\hline \multirow{4}{*}{-0.28} & -0.31 & 3.18 \\
\hline & -1.17 & 2.32 \\
\hline & -0.48 & 3.01 \\
\hline & -0.49 & 3.00 \\
\hline
\end{tabular}

5.21

3.97

5.41

4.02

3.54

4.46

5.14

4.87

4.82

4.95

3.45

$-1.21$

$-0.19-0.16 \quad-0.25$

$\begin{array}{lll}-0.17 & -0.13 & -0.28\end{array}$

$$
-0.44^{2}
$$

$-0.53$

$-1.90$

1.59

4.70

$-0.65 \quad 2.84$

$\begin{array}{ll}-0.65 & 2.86 \\ -0.63 & -10\end{array}$

$-1.10 \quad 2.39$
-0.16

0.15

4.72

4.25

5.51

3.98

5.31

5.08

5.11

28

$196 \mathrm{a}-0.38$

$\begin{array}{rr}313 & -0.88 \\ 29 & -2.10\end{array}$

-1.32
-0.73

16

$-1.36$

26

455

$-0.79$

$-0.03$

$-0.41$

0.32

$\begin{array}{rrrrr} & -0.29 & & -0.04 & 3.45 \\ -0.19^{1} & -0.27 & & -0.27 & 3.22 \\ -0.16^{1} & -0.25 & -0.39 & -0.24 & 3.25 \\ & -0.22 & -0.26 & -0.20 & 3.29\end{array}$

$-1.37$

$-0.27$

3.45

$\begin{array}{ll}-0.38 & 3.11\end{array}$

4.97

$-0.88$

$-2.10$

1.39

$\begin{array}{ll}-1.32 & 2.17\end{array}$

$-0.73 \quad 2.76$

$-1.36 \quad 2.13$

$\begin{array}{ll}-0.79 & 2.70\end{array}$

$0.14 \quad 3.63$

$53-2.55$

$-2.55$

3.08

$\begin{array}{ll} & -1.18 \\ 456 & -1.47\end{array}$

$165-0.96$

$\begin{array}{ll}28 & -0.69\end{array}$

$578 \mathrm{a}-1.24$

$\begin{array}{rrr}29 & -1.98 & \\ 194 & 0.20 & 0.07 \\ & -0.83 & \\ 194 & 0.12 & 0.01\end{array}$

$-0.88$

102

$-1.19$

$\begin{array}{rrrr}164 & -0.88 & & \\ & -1.22 & & \\ 165 & -1.28 & & \\ 28 & -0.52 & -0.70 & -0.65 \\ 54 & -1.47 & & \end{array}$ $\begin{array}{lll}-1.18 & 2.31 & 4.16\end{array}$

$\begin{array}{ll}-1.47 & 2.02 \\ -\end{array}$

$-0.96 \quad 2.53$

$\begin{array}{llll}-0.73^{2} & -1.07 & -0.82 & 2.67 \\ & & -1.24 & 2.25\end{array}$

3.87

4.38

4.52

3.16

-2.39
-0.09

$-2.18 \quad 1.31$

$\begin{array}{ll}0.06 & 3.55 \\ -0.83 & 2.66\end{array}$

$\begin{array}{rr}-0.83 & 2.66 \\ -1.00 & 3.49\end{array}$

$\begin{array}{ll}-1.19 & 2.30\end{array}$

5.40

5.34

4.15

$-0.88 \quad 2.61$

$\begin{array}{ll}-1.22 & 2.27\end{array}$

$\begin{array}{ll}-1.28 & 2.21\end{array}$

$-0.76 \quad 2.73$

4.46

4.12

4.06

4.58

3.87 
TABI.E 3.-Oscillator Strengths for Ultraviolet Lines of Fe I-Continued

Wave-

Low $\therefore$ Energy Levels e.p.

3129.178

3129.335

3132.514

3134.112

3135.590

3135.863

3139.661

3140.391

3142.453

3142:891

3143.243

3143.990

3144.488

3144.924

3145.057

3146.475

3147.793

3148.178

3148.408

3149.492

3150.304

3151.353

3151.867

3153.200

3154.505

3155.134

3155.293

3155.796

3156.275

3156.464

3157.040

3157.880

3158.990

3159.248

3159.437

3160.200

3160.344

3160.658

3161.373

3161.558

3161.949

3162.335

3164.308

3165.005

3165.860

3166.435

3166.596

3166.982

3167.907

3168.858

3171.353

3171.663

3172.067

3172.292

3173.400

3173.663

3174.222

3175.447

3175.970

3176.366

$\begin{array}{rr}19757-51705 & 2.45 \\ 11976-43923 & 1.48 \\ 25900-57814 & 3.21 \\ 7728-39626 & 0.96 \\ 21999-53882 & 2.73\end{array}$

$19788-51668 \quad 2.45$

$19351-51192 \quad 2.40$

$26140-57974 \quad 3.24$

$19757-51570 \quad 2.45$

$18378-50187 \quad 2.28$

$0-31805 \quad 0.00$

$25900-57698 \quad 3.21$

$19621-51409 \quad 2.43$

$24119-55906 \quad 2.99$

$24339-56098 \quad 3.02$

$22249-54005 \quad 2.76$

$19621-51374 \quad 2.43$

$23784-55526 \quad 2.95$

$26479-58213 \quad 3.28$

$21999-53722 \quad 2.73$

$416-32134 \quad 0.05$

$19757-51462 \quad 2.45$

$20020-51705 \quad 2.48$

$19621-51305 \quad 2.43$

$19390-51069 \quad 2.40$

$26140-57814 \quad 3.24$

$24119-55791 \quad 2.99$

$19562-51229 \quad 2.42$

$19913-51570 \quad 2.47$

$23784-55430 \quad 2.95$

$21039-52683 \quad 2.61$

$22249-53892 \quad 2.76$

$26340-57974 \quad 3.26$

$19390-51023 \quad 2.40$

$19562-51192 \quad 2.42$

$12561-44184 \quad 1.56$

$19788-51409 \quad 2.45$

$19351-50968 \quad 2.40$

$19757-51370 \quad 2.45$

$19757-51351 \quad 2.45$

$19562-51149 \quad 2.42$

$19757-51335 \quad 2.45$

$20641-52213 \quad 2.56$

$17727-49298 \quad 2.20$

$24339-55906 \quad 3.02$

$26140-57698 \quad 3.24$

$19913-51461 \quad 2.47$

$11976-43500 \quad 1.48$

$20020-51540 \quad 2.48$

$17727-49243 \quad 2.20$

$22249-53763 \quad 2.76$

$23245-54748 \quad 2.88$

$17727-49227 \quad 2.20$

$26479-57974 \quad 3.28$

$19351-50833 \quad 2.40$

$23270-54748 \quad 2.88$

$21039-52512 \quad 2.61$
$19913-51705 \quad 2.47$

$19562-51335 \quad 2.42$

$19913-51604 \quad 2.47$
Mult. Arc Spk $\mathrm{CW}(\mathrm{N})$ HC $\mathrm{R}(\mathrm{N})$ KOC $\mathrm{MS}$ Best $\log \operatorname{gf} \lambda$

$\log$ No.

\begin{tabular}{|c|c|c|c|}
\hline 161 & -0.72 & & \\
\hline 52 & -0.76 & -1.04 & \\
\hline 578 & 0.08 & -0.10 & \\
\hline 28 & -0.92 & -1.05 & \\
\hline 194 & -0.97 & & \\
\hline 155 & -0.90 & -0.47 & \\
\hline 578 & 0.19 & -0.07 & \\
\hline 164 & 0.04 & -0.25 & \\
\hline 144 & -0.09 & -0.20 & \\
\hline 7 & -2.67 & & \\
\hline 578 & 0.47 & 0.19 & \\
\hline 161 & -0.18 & -0.37 & \\
\hline 195 & -1.46 & & \\
\hline 455 & -0.29 & -0.13 & \\
\hline 160 & -1.50 & & \\
\hline 455 & $\begin{array}{l}-0.50 \\
-1.22\end{array}$ & -0.24 & $-0.30^{1}$ \\
\hline $\begin{array}{l}194 \\
453\end{array}$ & $\begin{array}{l}-0.65 \\
-1.49\end{array}$ & -0.38 & $-0.63^{1}$ \\
\hline $578 \mathrm{a}$ & -0.13 & -0.24 & $0.02^{1}$ \\
\hline 311 & 0.41 & & $0.45^{1}$ \\
\hline 7 & -2.91 & & \\
\hline 161 & 0.23 & -0.06 & $0.10^{1}$ \\
\hline 161 & -0.17 & & $-0.31^{1}$ \\
\hline 161 & -1.26 & & \\
\hline 193 & -0.52 & -0.50 & $-0.63^{1}$ \\
\hline $192 a$ & -1.35 & & \\
\hline 578 & 0.30 & 0.05 & $0.40^{1}$ \\
\hline 454 & -0.64 & -0.32 & \\
\hline 160 & 0.36 & 0.06 & $0.35^{1}$ \\
\hline 164 & 0.26 & -0.10 & $0.26^{1}$ \\
\hline 452 & -0.89 & & \\
\hline 259 & -1.43 & & \\
\hline
\end{tabular}

$\begin{array}{lll}-0.72 & 2.78 & 4.62 \\ -0.01 & 3.49 & 5.33 \\ -1.12 & 2.38 & 4.22 \\ -0.92 & 2.58 & 4.41\end{array}$

$-0.97$

2.53

$-0.66^{1}$

$\begin{array}{rrr}0.06 & 3.56 & 5.39 \\ -0.17 & 3.33 & 5.16\end{array}$

$-0.30$

$\begin{array}{lll}-0.26 & 3.24 & 5.07\end{array}$

$\begin{array}{lll}-3.20 & 0.30 & 2.13\end{array}$

$\begin{array}{lll}0.33 & 3.83 & 5.66\end{array}$

$\begin{array}{lll}-0.28 & 3.22 & 5.05\end{array}$

$\begin{array}{lll}-1.46 & 2.04 & 3.87\end{array}$

$\begin{array}{lll}-0.21 & 3.29 & 5.12\end{array}$

$\begin{array}{lll}-1.50 & 2.00 & 3.83\end{array}$

$\begin{array}{lll}-0.35 & 3.15 & 4.98\end{array}$

$\begin{array}{lll}-1.22 & 2.28 & 4.11\end{array}$

$\begin{array}{lll}-0.55 & 2.95 & 4.78\end{array}$

$\begin{array}{lll}-1.49 & 2.01 \quad 3.84\end{array}$

$\begin{array}{lll}-0.12 & 3.38 & 5.21\end{array}$

$\begin{array}{lll}0.43 & 3.93 & 5.76\end{array}$

$\begin{array}{lll}-2.91 & 0.59 & 2.42\end{array}$

$\begin{array}{rrr}0.02 & 3.52 & 5.35 \\ -0.24 & 3.26 & 5.09\end{array}$

$-0.18$

$\begin{array}{lll}-1.26 & 2.24 & 4.07\end{array}$

$\begin{array}{lll}-0.55 & 2.95 & 4.78\end{array}$

$\begin{array}{lll}-1.35 & 2.15 & 3.98\end{array}$

$\begin{array}{lll}0.25 & 3.75 & 5.58\end{array}$

$\begin{array}{lll}-0.48 & 3.02 & 4.85\end{array}$

$\begin{array}{lll}0.21 & 3.71 & 5.54\end{array}$

$\begin{array}{lll}0.06 & 3.56 & 5.39\end{array}$

$\begin{array}{lll}-0.89 & 2.61 & 4.44\end{array}$

$\begin{array}{lll}-1.43 & 2.07 & 3.90\end{array}$

$\begin{array}{lll}-1.38 & 2.12 \quad 3.95\end{array}$

$\begin{array}{lrrr}578 & 0.00 & -0.08 & \\ 192 \mathrm{a} & -0.37 & -0.22 & \\ 155 & 0.46 & 0.14 & 0.39^{1} \\ 52 & -0.94 & -1.09 & -0.96^{1} \\ 195 & -1.25 & & \end{array}$

$\begin{array}{lll}-0.04 & 3.46 & 5.28\end{array}$

$\begin{array}{lll}-0.30 & 3.20 & 5.02\end{array}$

$\begin{array}{lll}0.29 & 3.79 & 5.61\end{array}$

$\begin{array}{lll}-1.25 & 2.25 & 4.07\end{array}$

$\begin{array}{llll}160 & 0.16 & 0.13 & 0.13\end{array}$

$\begin{array}{lll}0.07 & 3.57 & 5.39\end{array}$

$\begin{array}{rrr}0.07 & 2.96 & 4.78\end{array}$

$\begin{array}{lll}-0.73 & 2.77 & 4.59\end{array}$

$\begin{array}{lll}-0.36 & 3.14 & 4.96\end{array}$

$155-0.24 \quad-0.43 \quad-0.32^{1}$

$-0.47^{1}$

$\begin{array}{llll}160 & -0.01 & -0.18 & -0.06^{1}\end{array}$

$-0.29$

$-0.14 \quad 3.36$

5.18

$\begin{array}{lrrrr}259 & 0.28 & 0.21 & 0.22^{1} & 0.15\end{array}$

$\begin{array}{lll}0.22 & 3.72 & 5.54\end{array}$

$-0.01 \quad 3.49 \quad 5.31$

$\begin{array}{lll}-1.51 & 1.99 & 3.81\end{array}$

$\begin{array}{lll}0.13 & 3.63 & 5.45 \\ -0.32 & 3.18 & 5.00\end{array}$

$0.19^{1}$
$-0.21^{1}$

$\begin{array}{lll}-0.32 & 3.18 & 5.00\end{array}$

$\begin{array}{lll}-0.86 & 2.64 & 4.46\end{array}$

$\begin{array}{lll}-0.59 & 2.91 \quad 4.73\end{array}$

$\begin{array}{lll}-0.60 & 2.90 & 4.72\end{array}$

$\begin{array}{lll}-1.27 & 2.23 & 4.05\end{array}$

$\begin{array}{lll}-0.55 & 2.95 & 4.77\end{array}$

$\begin{array}{lll}-0.52 & 2.98 & 4.80\end{array}$

$\begin{array}{lll}-1.45 & 2.05 & 3.87\end{array}$

$\begin{array}{lll}0.32 & 3.82 & 5.64\end{array}$

$\begin{array}{lll}-0.87 & 2.63 & 4.45\end{array}$

$\begin{array}{lll}-0.40 & 3.10 & 4.92\end{array}$ 
TABLE 3.-Oscillator Strengths for Ultraviolet Lines of Fe I-Continued

Wave $\stackrel{1}{A} \quad \underset{K}{ } \quad$ Volts

$\begin{array}{lll}3178.015 & 19351-50808 & 2.40 \\ 3178.545 & 24339-55791 & 3.02 \\ 3178.967 & 19621-51069 & 2.43 \\ 3179.479 & 12969-44411 & 1.6 \\ 3180.223 & 19757-51192 & 2.4\end{array}$

$\begin{array}{lll}3180.756 & 704-32134 & 0.09\end{array}$

3181.522

3181.922

3182.060

3182.980

3183.582

3184.112

3184.622

3184.896

3187.171

3188.026

3188.567

3188.819

3190.020

3190.651

3190.816

3191.116
3191.660

3192.417

3192.799

3193.227

3193.303

3194.422

3196.147

3196.930

3197.521

3198.266

3199.530

3200.475

3200.785

3202.562

3205.400

3207.089

3207.649

3208.470

3209.297

3210.230

3210.830

3211.487

3211.683

3211.872

3211.989

3214.044

3214.396

3214.624

3215.940

3217.380

3219.581

3219.806

3221.931

3222.069

3223.273

3223.480

3223.844

3225.789 $\begin{array}{rr}704-32134 & 0.09 \\ 20875-52297 & 2.59 \\ 24336-55754 & 3.02 \\ 19562-50980 & 2.42 \\ 17727-49135 & 2.20\end{array}$

$19621-51023 \quad 2.43$

$26875-58272 \quad 3.33$

$19757-51149 \quad 2.45$

$416-31805 \quad 0.05$

$23245-54612 \quad 2.88$

$21999-53358 \quad 2.73$

$19351-50704 \quad 2.40$

$20020-51370 \quad 2.48$

$20875-52213 \quad 2.59$

$24575-55907 \quad 3.05$

$24575-55906 \quad 3.05$

$20641-51969 \quad 2.56$

$0-31323 \quad 0.00$

$17927-49243 \quad 2.22$

$20020-51331 \quad 2.48$

$19913-51219 \quad 2.47$

$19913-51208 \quad 2.47$

$22846-54125 \quad 2.83$

$19562-50833 \quad 2.42$

$27560-58825 \quad 3.42$

$21039-52297 \quad 2.61$

$19562-50808 \quad 2.42$

$\begin{array}{ll}19913-51149 & 2.47\end{array}$

$704-31937 \quad 0.09$

$24575-55791 \quad 3.05$

$20020-51208 \quad 2.48$

$19351-50523 \quad 2.40$

$22838-54005 \quad 2.83$

$27666-58825 \quad 3.43$

$27560-58710 \quad 3.42$

$19562-50704 \quad 2.42$

$\begin{array}{ll}19913-51048 & 2.47\end{array}$

$20020-51149 \quad 2.48$

$17927-49053 \quad 2.22$

$19351-50475 \quad 2.40$

$19757-50862 \quad 2.45$

$\begin{array}{ll}704-31805 & 0.09\end{array}$

$18378-49477 \quad 2.28$

$19913-50999 \quad 2.47$

$19351-50423 \quad 2.40$

$19757-50808 \quad 2.45$

$\begin{array}{ll}19562-50611 & 2.42\end{array}$

$20020-51048 \quad 2.48$

$19351-50378 \quad 2.40$

$11976-42992 \quad 1.48$

$26628-57641 \quad 3.30$

$\begin{array}{ll}7986-38996 & 0.99\end{array}$

$19351-50342 \quad 2.40$
$0-31307 \quad 0.00$

$26875-58002 \quad 3.33$
Mult.
No.

Arc Spk CW(N) HC

$\mathrm{HC}$

R(N) KOC MS Best $\log$ gf $\lambda$

$\log$

\begin{tabular}{|c|c|c|c|c|c|c|c|c|c|c|}
\hline 156 & 0.32 & 0.01 & $0.33^{1}$ & & $0.16^{1}$ & 0.06 & & 0.18 & 3.68 & 5.50 \\
\hline 454 & -0.42 & -0.24 & & & & & & -0.33 & 3.17 & 4.99 \\
\hline $192 a$ & -0.48 & -0.35 & $-1.21^{1}$ & & & & & -0.42 & 3.08 & 4.90 \\
\hline 52 & -1.14 & & & & & & & -1.14 & 2.36 & 4.18 \\
\hline 155 & 0.77 & & $0.74^{2}$ & & $0.66^{1}$ & 0.54 & & 0.69 & 4.19 & 6.01 \\
\hline 7 & -2.10 & -2.19 & & & & -2.48 & & -2.26 & 1.24 & 3.06 \\
\hline 258 & -0.06 & 0.06 & & & & & & 0.00 & 3.50 & 5.32 \\
\hline 505 & -0.13 & & & & $-0.52^{1}$ & & & -0.32 & 3.18 & 5.00 \\
\hline 159 & -0.22 & -0.42 & & & & & & -0.32 & 3.18 & 5.00 \\
\hline 100 & -0.25 & & $-0.25^{1}$ & & & & & -0.25 & 3.25 & 5.07 \\
\hline $192 a$ & -1.22 & & & & & & & -1.22 & 2.28 & 4.10 \\
\hline 711 & -0.72 & & & & & & & -0.72 & 2.78 & 4.59 \\
\hline 155 & -0.41 & -0.52 & & & $-0.49^{1}$ & & & -0.47 & 3.03 & 4.84 \\
\hline 7 & -1.80 & -1.99 & $-1.87^{2}$ & & $-2.29^{1}$ & -2.15 & & -2.00 & 1.50 & 3.31 \\
\hline 333 & -0.95 & & & & & & & -0.95 & 2.55 & 4.36 \\
\hline & -0.63 & & & & & & & -0.63 & 2.87 & 4.68 \\
\hline 159 & -0.09 & -0.19 & & & & -0.36 & & -0.21 & 3.29 & 5.10 \\
\hline 159 & 0.29 & 0.05 & & & & 0.01 & & 0.12 & 3.62 & 5.43 \\
\hline 259 & -0.74 & -0.60 & $-0.61^{1}$ & & & & & -0.65 & 2.85 & 4.66 \\
\hline 548 & -0.23 & -0.07 & & & & & & -0.15 & 3.35 & 5.16 \\
\hline 548 & -0.28 & 0.12 & & & & & & -0.08 & 3.42 & 5.23 \\
\hline 258 & -0.72 & & & & & & & -0.72 & 2.78 & 4.59 \\
\hline 8 & -1.80 & -1.99 & $-1.86^{2}$ & & $-2.29^{1}$ & -2.19 & & -2.00 & 1.50 & 3.31 \\
\hline 100 & -0.96 & & & & & & & -0.96 & 2.54 & 4.35 \\
\hline 155 & 0.58 & & $0.43^{1}$ & & $0.48^{1}$ & 0.35 & & 0.46 & 3.96 & 5.77 \\
\hline 7 & & -1.41 & $-1.68^{1}$ & & $-1.99^{1}$ & -1.85 & -1.74 & -1.73 & 1.77 & 3.58 \\
\hline 159 & & 0.47 & & & & & & 0.47 & 3.97 & 5.78 \\
\hline 155 & -0.21 & -0.28 & $-0.07^{1}$ & & $-0.29^{1}$ & & & -0.21 & 3.29 & 5.10 \\
\hline 333 & 0.13 & & $0.24^{1}$ & & & & & 0.18 & 3.68 & 5.49 \\
\hline 155 & 1.04 & 0.77 & $1.00^{2}$ & & $1.01^{1}$ & & 0.74 & 0.93 & 4.43 & 6.24 \\
\hline 711 & -0.43 & & & & & & & -0.43 & 3.07 & 4.88 \\
\hline 258 & -1.03 & & & & & & & -1.03 & 2.47 & 4.28 \\
\hline 156 & 0.64 & 0.29 & $0.67^{2}$ & & $0.57^{1}$ & & & 0.57 & 4.08 & 5.88 \\
\hline 155 & 0.73 & 0.52 & $0.81^{2}$ & & $0.70^{1}$ & 0.41 & & 0.66 & 4.17 & 5.97 \\
\hline 8 & -2.59 & & & -2.89 & & -2.96 & & -2.81 & 0.70 & 2.50 \\
\hline 547 & -0.09 & -0.01 & $-0.02^{1}$ & -0.08 & & & & -0.05 & 3.46 & 5.26 \\
\hline 155 & 0.69 & 0.42 & $0.67^{2}$ & & $0.62^{1}$ & & & 0.60 & 4.11 & 5.91 \\
\hline 159 & -0.50 & & $-0.51^{1}$ & -0.79 & & & & -0.60 & 2.91 & 4.71 \\
\hline 382 & -1.45 & & & -1.60 & & & & -1.52 & 1.99 & 3.79 \\
\hline
\end{tabular}

$\begin{array}{lllll}159 & 0.30 & 0.03 & 0.34^{2} & 0.25 \\ 156 & 0.59 & 0.36 & 0.54^{2} & 0.48\end{array}$

$\begin{array}{ll}162 & 0.09\end{array}$

0.03

-0.22
1.03

$0.53^{1}$

0.25

3.76

$0.51 \quad 4.02$

5.55

0.91

0.77

-0.03
0.90

3.48
4.41

5.27

6.20

$98-0.02$

158

156

7

0.73

1.02

$0.70^{1} \quad-0.17$

$-0.02$

3.49

0.72

$1.03 \quad 4.54$

$-1.82 \quad 1.69$

$-1.38$

2.13

5.28

6.02

6.33

3.48

156

157

156

158

156

0.59

0.45

$0.57^{2}$

0.41

0.41
0.75
0.57

0.22

$0.37^{2}$

$-1.38$

0.57

0.44

$0.63^{1}$

$0.64^{1}$

0.56

0.36

0.68

0.46

4.07

$0.68 \quad 0.74^{1}$

0.45

$-0.98^{1}$

$-0.98$

4.19

3.97

2.53

$\begin{array}{llllll}156 & 1.05 & 0.92 & 1.09^{2} & 1.11 & 1.09^{1}\end{array}$

1.06

4.57

$\begin{array}{rr}-1.89 & 1.62\end{array}$

$-0.97-0.38$

$27-1.19$

1.29

1.07

$1.20^{2}$

1.27

$1.26^{1}$

3.92

5.86

5.66

5.98

5.76

6.36

3.41

4.33

4.11
6.52 
TABLE 3.-Oscillator Strengths for Ultraviolet Lines of Fe I-Continued

Wave- Low

length Energy Levels e.p.

$\AA \quad K \quad$ Volts

Mult. No.

3226.714
3227.063
3227.798
3228.003
3228.254

$704-31686$

$20020-50999-0.09$

$19562-50534 \quad 2.42$

$22838-53808 \quad 2.83$

$19913-50880 \quad 2.47$

3228.900

3229.122

3229.595

3229.994

3230.210

3230.963

3231.576

3232.155

3233.053

3233.967

$20020-50981 \quad 2.48$

$978-31937 \quad 0.12$

22846 - $53801 \quad 2.83$

$24575-55526 \quad 3.05$

$19913-50861 \quad 2.47$

$19757-50699 \quad 2.45$

$11976-42912 \quad 1.48$

$21039-51969 \quad 2.61$

$26106-57028 \quad 3.24$

$19562-50475 \quad 2.42$

3234.614

3235.312

3235.592

3235.833

3236.223

3237.234

3238.313

3238.535

3239.029

3239.436

3240.013

3240.122

3241.502

3242.268

3243.109

3243.406

3244.190

3246.005

3246.482

3246.962

416-31323 0.05

$21999-52899 \quad 2.73$

$21716-52613 \quad 2.69$

$22838-53734 \quad 2.83$

$416-31307 \quad 0.05$

$20875-51756 \quad 2.59$

$24575-55446 \quad 3.05$

$24507-55376 \quad 3.04$

$18378-49243 \quad 2.28$

$19562-50423 \quad 2.42$

$24575-55430 \quad 3.05$

$19757-50611 \quad 2.45$

$8155-38996 \quad 1.01$

$20875-51708 \quad 2.59$

$19788-50614 \quad 2.45$

$26875-57698 \quad 3.33$

$19562-50378 \quad 2.42$

$888-31686 \quad 0.11$

$20875-51668 \quad 2.59$

$17727-48516 \quad 2.20$

3247.278

3248.206

3249.037

3249.19

$19913-50699 \quad 2.47$

$19757-50534 \quad 2.45$

$21999-52769 \quad 2.73$

$20641-51409 \quad 2.56$

$12561-43326 \quad 1.56$

3250.394

3250.625

3251.236

3252.916

3253.610

3253.834

3253.949

3254.363

3254.734

3257.244

3257.594

3259.991

3260.261

3261.332

3262.009

$23052-53808 \quad 2.86$

$17550-48305 \quad 2.18$

$17727-48476 \quad 2.20$

$20641-51374 \quad 2.56$

$26225-56951 \quad 3.25$

$20641-51365 \quad 2.56$

$21039-51762 \quad 2.61$

$26351-57070 \quad 3.27$

$21716-52431 \quad 2.69$

$24119-54811 \quad 2.99$

$17550-48239 \quad 2.18$

$19757-50423 \quad 2.45$

$20641-51305 \quad 2.56$

$27560-58213 \quad 3.42$

$27167-57814 \quad 3.37$

3263.370

$19552-50187 \quad 2.42$

3264.512

3264.710

3265.047

$17727-48351 \quad 2.20$

$19913-50534 \quad 2.47$

$\begin{array}{ll}704-31323 & 0.09\end{array}$

$17550-48163 \quad 2.18$

9 $\begin{array}{llllll}\text { Arc Spk } & \mathrm{CW}(\mathrm{N}) \mathrm{HC}(\mathrm{N}) \text { KOC MS Best }\end{array}$ $-\log g f$

-

$\log \operatorname{gf} \lambda \operatorname{gA} / \lambda$

\begin{tabular}{|c|c|c|c|c|c|}
\hline 8 & -3.13 & & & -3.44 & \\
\hline 156 & -0.43 & & $-0.29^{1}$ & -0.76 & $-0.38^{1}$ \\
\hline 157 & 0.80 & & & 0.69 & \\
\hline 379 & 0.19 & & & -0.26 & \\
\hline 157 & 0.23 & 0.12 & & -0.03 & \\
\hline 157 & -0.18 & -0.32 & & -0.34 & \\
\hline 8 & -2.26 & -2.31 & & -2.28 & \\
\hline 333 & -0.63 & -0.52 & & & \\
\hline 546 & 0.28 & 0.37 & & & \\
\hline 158 & 0.27 & 0.00 & & -0.08 & \\
\hline $\begin{array}{r}157 \\
50\end{array}$ & 0.47 & 0.21 & $0.47^{2}$ & 0.29 & \\
\hline 258 & -1.66 & & & & \\
\hline 620 & 0.68 & 0.91 & $0.93^{1}$ & 0.94 & \\
\hline
\end{tabular}

$\begin{array}{rrr}-3.28 & 0.23 & 2.02 \\ -0.37 & 3.14 & 4.93 \\ 0.74 & 4.25 & 6.04 \\ 0.19 & 3.70 & 5.49\end{array}$

$\begin{array}{lll}0.11 & 3.62 & 5.41\end{array}$

$\begin{array}{lll}-0.28 & 3.23 & 5.02\end{array}$

$\begin{array}{lll}-2.28 & 1.23 & 3.02\end{array}$

$\begin{array}{lll}-0.58 & 2.93 & 4.72\end{array}$

$\begin{array}{lll}0.32 & 3.83 & 5.62\end{array}$

$\begin{array}{lll}0.06 & 3.57 & 5.36\end{array}$

$\begin{array}{lll}0.38 & 3.89 & 5.68\end{array}$

$\begin{array}{lll}-1.57 & 1.94 & 3.73\end{array}$

$\begin{array}{lll}-1.66 & 1.85 & 3.64\end{array}$

$\begin{array}{lll}0.86 & 4.37 & 6.16\end{array}$

$\begin{array}{lll}0.41 & 3.92 & 5.70\end{array}$

$\begin{array}{lll}-2.12 & 1.39 & 3.17\end{array}$

$\begin{array}{lll}-1.93 & 1.58 & 3.36\end{array}$

$\begin{array}{lll}-1.24 & 2.27 & 4.05\end{array}$

$\begin{array}{lll}-1.37 & 2.14 & 3.92\end{array}$

$-1.11$

-1.37
-1.88

$\begin{array}{lll}-1.91 & -1.96^{1} & -1.77\end{array}$

$\begin{array}{ll}-1.88 & 1.63\end{array}$

$\begin{array}{lll}-1.54 & 1.97 & 3.75\end{array}$

$\begin{array}{lll}-1.52 & 1.99 & 3.77\end{array}$

$\begin{array}{lll}-1.36 & 2.15 & 3.93\end{array}$

$\begin{array}{rrr}-1.20 & 2.31 & 4.09 \\ 0.77 & 4.28 & 6.06\end{array}$

$\begin{array}{lll}-1.33 & 2.18 & 3.96\end{array}$

$\begin{array}{lll}-1.28 & 2.23 & 4.01\end{array}$

$\begin{array}{lll}-3.26 & 0.25 & 2.03\end{array}$

$\begin{array}{lll}-1.50 & 2.01 & 3.79\end{array}$

$-3.26$

$-1.46$

$-1.53$

$-1.03$

$-0.98 \quad 2.53$

4.31

$\begin{array}{lll}-0.02 & 3.49 & 5.27\end{array}$

$\begin{array}{lll}0.71 & 4.22 & 6.00\end{array}$

$\begin{array}{lll}-2.08 & 1.43 & 3.21\end{array}$

$\begin{array}{lll}-0.39 & 3.12 & 4.90\end{array}$

$\begin{array}{lll}-0.11 & 3.40 & 5.18\end{array}$

$\begin{array}{lll}-0.18 & 3.33 & 5.11\end{array}$

$\begin{array}{lll}0.27 & 3.78 & 5.56\end{array}$

$\begin{array}{lll}-1.39 & 2.12 & 3.90\end{array}$

$\begin{array}{lll}-0.54 & 2.97 & 4.75\end{array}$

$\begin{array}{lllll}253 & -0.56 & -0.34 & -0.68^{1} & -0.56\end{array}$

$-2.52$

$379-0.65$

252

681

$-0.41$

$-0.23$

$-0.31$

0.10

0.21

0.21
-0.59

$\begin{array}{lll}-0.45 & -0.391 & -0.59 \\ -0.15 & -0.08^{1} & -0.65\end{array}$

$\begin{array}{lll}-0.18 & -0.32^{1} & -0.37\end{array}$

$0.14^{1}-0.07$

250

257

620

308

$\begin{array}{rrrr} & & & -0.85 \\ -0.69 & -0.33 & & -0.82 \\ 0.67 & 0.90 & 0.82^{1} & 0.83 \\ -0.92 & & & -1.14\end{array}$

$-0.65$

$-0.46$

$-0.15$

$-0.30$

0.13

$-0.85$

$-0.51$

0.80

$-1.03$

$-0.25$

$-0.16$

$-0.30$

$-0.48$

$-0.26$

$-0.14$

$-0.71$

$-0.36$

$-0.78$

-2.18
0.46

$0.42^{1}$

-2.14
0.51

$\begin{array}{ll}2.86 & 4.64\end{array}$

$3.05 \quad 4.83$

$\begin{array}{ll}3.36 & 5.14\end{array}$

$3.21 \quad 4.99$

$3.64 \quad 5.42$

$2.66 \quad 4.44$

$3.00-4.78$

$\begin{array}{ll}4.31 & 6.09\end{array}$

$2.48 \quad 4.26$

$3.35-5.13$

$\begin{array}{ll}3.21 & 4.98\end{array}$

$3.03-4.80$

$\begin{array}{ll}3.25 & 5.02\end{array}$

5.14

4.57

$\begin{array}{ll}2.80 & 4.57 \\ 2.15 & 4.92\end{array}$

$\begin{array}{ll}2.73 & 4.50\end{array}$

$\begin{array}{ll}1.33 & 3.10\end{array}$ 
TABLE 3.-Oscillator Strengths for Ultraviolet Lines of Fe I-Continued

Wave-

- Low

Energy Levels Low

A $\mathrm{K} \quad$ Volts

3268.234

$17927-48516 \quad 2.22$

(2)

$27395-57974-3.40$

3269.416

$17727-48305$

2.20

3269.964

$17550-48123$

2.18

$3271.001 \quad 17727-48290 \quad 2.20$

$3271.487 \quad 26225-56783 \quad 3.25$

$3271.684 \quad 11976-42533 \quad 1.48$

$\begin{array}{lll}3272.596 & 12561-43109 & 1.56\end{array}$

$3272.710 \quad 27666-58213 \quad 3.43$

3274.227

$17927-48460$

2.22

3274.453

3275.685

$27167-57698 \quad 3.37$

3275.848

3276.471

3278.741

3279.739

3280.26

3280.763

3281.824

3282.720

3282.89

3283.430

3284.589

3285.200

3286.022

$3286.444 \quad 27395-57814 \quad 3.40$

$\begin{array}{lll}3286.4454 & 27395-57814 & 3.40 \\ 3286.754 & 17550-47967 & 2.18\end{array}$

$3287.117 \quad 23711-54125 \quad 2.94$

$3288.651 \quad 19552-49951 \quad 2.42$

3288.967

3289.44

3290.714

3292.022

3292.591

3293.142

3296.467

3296.806

3298.133

3298.537

3299.077

3301.227

3301.917

3303.574

3304.346

3305.972

3306.356

3306.490

3307.008

3307.234

3308.76

3310.347

3310.496

3311.45

3312.224

3313.723

3314.070

3314.441

3314.742
$17727-48123 \quad 2.20$

$22838-53230 \quad 2.83$

$17550-47930 \quad 2.18$

$17927-48305 \quad 2.22$

$26225-56593 \quad 3.25$

$17927-48290 \quad 2.22$

$12969-43326 \quad 1.61$

$20875-51201 \quad 2.59$

$26628-56951 \quad 3.30$

$17927-48239 \quad 2.22$

$27666-57974 \quad 3.43$

$27395-57698 \quad 3.40$

$12561-42860 \quad 1.56$

$22947-53230 \quad 2.84$

$26106-56383 \quad 3.24$

$24339-54600 \quad 3.02$

$27560-57814 \quad 3.42$

$17727-47967 \quad 2.20$

$17927-48163 \quad 2.22$

$26624-56859 \quad 3.30$

$23784-54014 \quad 2.95$

$26106-56334 \quad 3.24$

$19390-49604 \quad 2.40$

$23784-53983 \quad 2.95$

$26225-56423 \quad 3.25$

$7986-38175 \quad 0.99$

$24119-54301 \quad 2.99$

$12969-43138 \quad 1.61$

$27543-57709 \quad 3.41$

$21039-51201 \quad 2.61$

$26624-56783 \quad 3.30$
Mult. Arc Spk CW(N) HC R(N) KOC MS Best No.

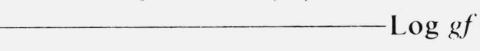

-

$\begin{array}{rrrrrr}95 & -0.41 & -0.38 & -0.38^{1} & -0.64 & \\ 710 & -0.52 & & -0.29^{1} & & \\ 95 & -1.94 & & & & \\ 90 & -1.67 & & & -0.57 & \\ 91 & 0.48 & 0.41 & 0.51^{2} & 0.54 & 0.46^{1} \\ & & & & & \\ 680 & -0.51 & -0.27 & & -0.37 & \\ 49 & -1.57 & & & -1.70 & \\ 95 & -1.98 & & & & \\ 712 & -1.04 & & -0.60^{1} & -0.73 & \\ 95 & -1.84 & & & & \\ 710 & -0.11 & -0.12 & -0.01^{1} & -0.09 & \\ 308 & -1.34 & & & -1.30 & \\ 450 \mathrm{a} & -1.53 & & & -1.58 \\ 90 & -0.54 & -0.54 & -0.65^{1} & -0.70 \\ 144 & -0.45 & -0.38 & -0.47^{1} & -0.56 \\ & & & & & \\ 449 & -0.69 & & & -0.84 \\ 620 & 0.66 & 0.81 & 0.81^{1} & 0.82 & \\ 451 & -2.17 & & & -1.07 & \end{array}$

$-0.45$

$-0.40$

$\log \mathrm{gf} \lambda$

$\log$

$50-2.38$

$449-1.35$

$\begin{array}{rrrrrr}680 & 0.21 & 0.24 & 0.12^{1} & 0.01 & \\ 27 & -2.99 & & & -2.26 & \\ 91 & -0.27 & -0.35 & -0.27^{1} & -0.46 & -0.27^{1} \\ 396 & -0.79 & & -0.05^{1} & -0.05 & \\ 90 & -0.92 & -0.41 & & -1.01 & \end{array}$

$-1.94$

0.48

3.06

3.11

1.57

1.84

$-0.38$

$-1.64$

$-1.98$

$-0.79$

(3.13

3.13

1.87

$-1.84$

1.53
2.72

2.72
1.68

$\begin{array}{ll}-0.08 & 3.44\end{array}$

$\begin{array}{ll}-1.32 & 2.20\end{array}$

$-1.56 \quad 1.96$
-0.61

$\begin{array}{ll}-0.61 & 2.91 \\ -0.46 & 3.06\end{array}$

$-0.46$

$-0.76$

0.78
-2.17
-2.38

2.76

$\begin{array}{ll}-2.38 & 1.14 \\ -1.35 & 2.17\end{array}$

4.30

1.35

0.14

$-2.99$

3.66

$-0.32$

0.53

$-0.96$

3.20

2.56

$\begin{array}{ll}-0.30 & 3.22\end{array}$

$\begin{array}{ll}0.85 & 4.37\end{array}$

$\begin{array}{ll}-0.57 & 2.95\end{array}$

$-1.01 \quad 2.51$
-0.86

$-0.86 \quad 2.66$

$-0.86 \quad 2.66$

$-1.30$

$-0.23$

2.66
3.29
4.18

$0.66 \quad 4.18$

$0.19 \quad 3.71$

$-2.06 \quad 1.46$

$\begin{array}{ll}-0.82 & 2.70\end{array}$

$\begin{array}{ll}-1.14 & 2.38\end{array}$

$-0.12 \quad 3.40$
-1.30

$-1.30 \quad 2.22$

$-0.92$

$-2.56$

-2.56
-0.77

$-0.44$

2.60

-0.92
-1.22

49
380
617

$-2.56$

$-0.54$

$-0.84$

$449-0.52$

$-0.69$

$710-1.42$

$\begin{array}{ll}91 & 0.6 \\ 91 & 0.74\end{array}$

$\begin{array}{rrrr}0.61 & 0.80^{1} & 0.72 & 0.82^{1} \\ 0.81 & 0.82^{1} & 0.94 & 0.87^{1}\end{array}$

$-0.60$

2.75

3.08

$-1.42-2.10$
-0.73

0.73

2.10
4.25

$0.84 \quad 4.36$

$\begin{array}{ll}0.82 & 3.30\end{array}$

$-0.80 \quad 2.72$

$\begin{array}{ll}680 & \\ 450 & -0.85\end{array}$

$-0.76$

0.44

$-1.43$

$-0.17$

$-0.10$

$-3.52$

$-1.22$

$-2.54$

$-1.02$

$-0.86$

0.71
$\mathrm{gA} / \lambda$

4.83

4.88

3.34

\begin{tabular}{l}
5.76 \\
\hline
\end{tabular}

4.90

3.64

3.30

4.49

5.20

3.96

3.72

4.67

4.52

6.06

3.11

2.90
3.93

5.42

2.28

4.95

4.31

4.97

6.12

4.70

4.26

4.41

3.97

5.04

5.93
5.46

3.21

4.45

4.13

5.15

3.97

4.35

2.71

4.50

4.67

3.85

6.00

6.11

5.05

5.71

3.83

5.09

5.16
1.74

4.04

2.72

4.24

4.40

5.97 
TABLE 3.-Oscillator Strengths for Ultraviolet Lines of Fe I-Continued

Wave-

length Energy Levels Low

A $\mathrm{K} \quad$ Volts

$3315.164 \quad 26628-56783 \quad 3.30$

3317.121

$18378-48516 \quad 2.28$

3319.258

3320.650

$24119-54237 \quad 2.99$

$19621-49727 \quad 2.43$

3320.779

3322.474

$24507-54612 \quad 3.04$

3323.738

3324.372

3324.538

3325.468

$23711-53801 \quad 2.94$

$22838-52916 \quad 2.83$

$26351-56423 \quad 3.27$

$19390-49461 \quad 2.40$

$19788-49851 \quad 2.45$

$3327.497 \quad 19390-49434 \quad 2.40$

$\begin{array}{lll}3327.961 & 17550-47590 & 2.18\end{array}$

$3328.867 \quad 26351-56383 \quad 3.27$

$3329.532 \quad 24575-54600 \quad 3.05$

$3329.970 \quad 24336-54357 \quad 3.02$

3330.316

3331.613

3331.778

3334.220

3334.278

3335.513

3335.770

3336.254

3337.666

3338.638

3339.195

3339.582

3340.567

3341.906

3342.216

3342.298

3343.240

3343.678

3345.679

3346.936

$24339-54357 \quad 3.02$

$19621-49628 \quad 2.43$

$20038-50043 \quad 2.48$

$19621-49604 \quad 2.43$

$26351-56334 \quad 3.27$

$12561-42533 \quad 1.56$

$22947-52916 \quad 2.84$

$26628-56593 \quad 3.30$

$21716-51668 \quad 2.69$

$24181-54125 \quad 3.00$

$19788-49727 \quad 2.45$

$24336-54271 \quad 3.02$

$18378-48305 \quad 2.28$

$21716-51630 \quad 2.69$

$18378-48290 \quad 2.28$

$22947-52858 \quad 2.84$

$17550-47453 \quad 2.18$

$24339-54237 \quad 3.02$

$19552-49433 \quad 2.42$

$17550-47420 \quad 2.18$

3347.507

3347.927

3349.739

3350.284

3351.524

3351.746

3352.929

3353.267

3354.064

3355.229

$24119-53983$

$18378-48239 \quad 2.28$

$22838-52683 \quad 2.83$

$19788-49628 \quad 2.45$

$17727-47556 \quad 2.20$

$21999-51826 \quad 2.73$

$19788-49604 \quad 2.45$

$19621-49434 \quad 2.43$

$23052-52858 \quad 2.86$

$26628-56423 \quad 3.30$

3355.517

$7728-37521$

0.96

3356.403

3356.695

3359.488

3359.814

3360.922

3361.959

3363.815

3364.639

3366.789

$18378-48163 \quad 2.28$

$24575-54357 \quad 3.05$

$6928-36686 \quad 0.86$

$26628-56383 \quad 3.30$

$19552-49298 \quad 2.42$

$22947-52683 \quad 2.84$

$22249-51969 \quad 2.76$

$\begin{array}{ll}20875-50587 & 2.59\end{array}$

$21716-51409 \quad 2.69$

3366.867

3367.159

3368.248

3368.983

$17727-47420 \quad 2.20$

$19552-49243 \quad 2.42$

$26225-55906 \quad 3.25$

$22838-52512 \quad 2.83$

$19788-49461 \quad 2.45$
Mult. Arc Spk $\mathrm{CW}(\mathrm{N})$ HC $\mathrm{R}(\mathrm{N})$ KOC MS Best

$\log$ No.

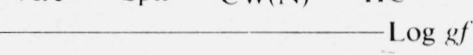

$\log g$

\begin{tabular}{|c|c|c|c|c|}
\hline 618 & -2.30 & & & \\
\hline 139 & -0.54 & -0.56 & $-0.61^{1}$ & -0.6 \\
\hline 449 & -0.37 & -0.31 & $-0.43^{1}$ & -0 . \\
\hline 190 & -0.89 & -0.61 & & \\
\hline 396 & -0.59 & -0.46 & & \\
\hline 396 & -0.18 & -0.37 & $-0.12^{1}$ & -0 . \\
\hline 379 & 0.22 & 0.35 & $0.30^{1}$ & \\
\hline 617 & -0.73 & & & -0 \\
\hline 191 & -0.41 & -0.34 & $-0.39^{1}$ & -0 . \\
\hline 191 & -0.43 & -0.41 & $-0.46^{1}$ & -0 \\
\hline 190 & -1.05 & -0.76 & $-1.09^{1}$ & \\
\hline 86 & -2.29 & & & \\
\hline 617 & 0.42 & 0.59 & $-1.59^{1}$ & 0 . \\
\hline \multirow[t]{2}{*}{$542 a$} & $\begin{array}{l}-0.68 \\
-1.31\end{array}$ & & & \\
\hline & -1.03 & & & \\
\hline 191 & -0.53 & -0.61 & $-0.61^{1}$ & -0 . \\
\hline 144 & -0.91 & -0.53 & & \\
\hline 190 & -1.22 & -0.64 & $-0.82^{1}$ & \\
\hline 617 & & & & \\
\hline
\end{tabular}

$\begin{array}{rll}-2.30 & 1.22 & 2.96 \\ -0.60 & 2.92 & 4.66 \\ -0.37 & 3.15 & 4.89 \\ -0.75 & 2.77 & 4.51 \\ -0.52 & 3.00 & 4.74 \\ -0.20 & 3.32 & 5.06 \\ 0.29 & 3.81 & 5.55 \\ -0.60 & 2.92 & 4.66 \\ -0.39 & 3.13 & 4.87 \\ -0.45 & 3.07 & 4.81 \\ -0.93 & 2.59 & 4.33 \\ -2.29 & 1.23 & 2.97 \\ 0.59 & 4.11 & 5.85 \\ -0.54 & 2.98 & 4.72 \\ -1.31 & 2.21 & 3.95 \\ -1.03 & 2.49 & 4.23 \\ -0.58 & 2.94 & 4.68 \\ -0.72 & 2.80 & 4.54 \\ -0.78 & 2.74 & 4.47 \\ -0.45 & 3.07 & 4.80\end{array}$

$\begin{array}{rrrrr}49 & -2.42 & -1.30 & & \\ 379 & -0.07 & -0.35 & -0.16^{1} & -0.07 \\ 618 & -0.31 & -0.02 & & -0.30 \\ 304 & -0.12 & -0.15 & -0.09^{1} & -0.10 \\ 396 & -0.44 & & -0.53^{1} & -0.53 \\ & & & & \\ 190 & -0.69 & -0.62 & -0.81^{1} & -0.68 \\ 502 & -0.70 & & & \\ 139 & -0.35 & -0.35 & -0.38^{1} & -0.25 \\ 303 & -0.24 & -0.21 & & -0.06 \\ 137 & -0.52 & -0.41 & -0.29^{1} & -0.31 \\ & & & & \\ 378 & -0.15 & -0.40 & & 0.32 \\ 88 & -1.95 & & & -0.78 \\ 449 & -0.79 & & & -0.76 \\ 141 & -2.40 & & & -1.02 \\ 87 & -1.24 & -0.97 & -1.38^{1} & -1.34 \\ & & & & \\ 449 & -1.50 & & & -0.83 \\ 138 & -0.44 & -0.43 & -0.45^{1} & -0.37 \\ 377 & -1.65 & & & \\ 191 & -1.36 & & & \\ 89 & -0.88 & -0.75 & & -0.79 \\ & & & & \\ 304 & -0.36 & -0.30 & -0.39^{1} & -0.40 \\ 190 & -1.45 & & & \\ 190 & -1.19 & -1.07 & & \\ 378 & -0.27 & -0.34 & -0.31^{1} & -0.04 \\ 617 & 0.45 & 0.53 & 0.59^{2} & 0.60\end{array}$

$-2.42$

$1.10 \quad 2.83$

$\begin{array}{lll}-0.16 & 3.36 & 5.09\end{array}$

$\begin{array}{lll}-0.21 & 3.31 & 5.04\end{array}$

$\begin{array}{lll}-0.12 & 3.40 & 5.13\end{array}$

$\begin{array}{lll}-0.50 & 3.02 \quad 4.75\end{array}$

$\begin{array}{lll}-0.70 & 2.82 & 4.55\end{array}$

$-0.70 \quad 2.82 \quad 4.55$

$\begin{array}{lll}-0.33 & 3.19 & 4.92\end{array}$

$\begin{array}{lll}-0.17 & 3.35 & 5.08\end{array}$

$\begin{array}{lll}-0.38 & 3.14 & 4.87\end{array}$

$\begin{array}{lll}-0.28 & 3.24 & 4.97\end{array}$

$\begin{array}{lll}-1.95 & 1.57 & 3.30\end{array}$

$\begin{array}{lll}-0.78 & 2.74 & 4.47\end{array}$

$\begin{array}{lll}-2.40 & 1.12 & 2.85\end{array}$

$\begin{array}{lll}-1.23 & 2.29 & 4.02\end{array}$

$\begin{array}{lll}-1.50 & 2.02 & 3.75\end{array}$

$\begin{array}{lll}-0.42 & 3.10 & 4.83\end{array}$

$\begin{array}{lll}-1.65 & 1.88 & 3.60\end{array}$

$\begin{array}{lll}-1.36 & 2.17 & 3.89\end{array}$

$\begin{array}{lll}-0.81 & 2.72 & 4.44\end{array}$

$\begin{array}{lll}-0.36 & 3.17 & 4.89\end{array}$

$\begin{array}{lll}-1.45 & 2.08 & 3.80\end{array}$

$\begin{array}{lll}-1.13 & 2.40 \quad 4.12\end{array}$

$\begin{array}{lll}-0.30 & 3.23 & 4.95\end{array}$

$\begin{array}{lll}0.55 & 4.08 & 5.80\end{array}$

$\begin{array}{lll}-3.14 & 0.39 & 2.11\end{array}$

$\begin{array}{lll}-0.60 & 2.93 & 4.65\end{array}$

$\begin{array}{lll}-0.78 & 2.75 & 4.47\end{array}$

$\begin{array}{lll}-2.08 & 1.45 & 3.17\end{array}$

$\begin{array}{lll}-0.35 & 3.18 & 4.89\end{array}$

$\begin{array}{rrrrr}25 & -2.05 & -2.01 & & -2.19 \\ 617 & -0.78 & -0.35 & -0.35^{1} & -0.35\end{array}$

$\begin{array}{lll}-1.30 & 2.23 & 3.94\end{array}$

$\begin{array}{lll}-0.97 & 2.56 & 4.27\end{array}$

$\begin{array}{lll}-1.30 & 2.23 & 3.94\end{array}$

$\begin{array}{lll}-1.56 & 1.97 & 3.68 \\ 0.19 & 3.72 & 5.43\end{array}$

0.19

$\begin{array}{lll}0.19 & 3.72 & 5.43\end{array}$

$\begin{array}{lll}-0.40 \quad 3.13 & 4.84\end{array}$

0.29

$\begin{array}{rrr}-0.40 & 3.13 & 4.84 \\ 0.29 & 3.82 & 5.53 \\ -0.89 & 2.64 & 4.35 \\ -1.63 & 1.90 & 3.61 \\ -1.31 & 2.22 & 3.93\end{array}$


TABLE 3.-Oscillator Strengths for Ultraviolet Lines of Fe I-Continued

Wave$\AA \quad K \quad$ Volts

$\begin{array}{ll}3370.785 & 21716-51374 \\ 3372.074 & 17550-47197\end{array}$

$3372.074 \quad 17550-47197$

$3372.352 \quad 24119-53763$

$3373.874 \quad 21999-51630$

$3374.176 \quad 17927-47556$

$3375.724 \quad 24119-53734$

$3378.676 \quad 21716-51305$

$3379.021 \quad 17550-47136 \quad 2.18$

$3380.112 \quad 22249-51826 \quad 2.76$

$\begin{array}{lll}3381.340 & 22947-52512 & 2.84\end{array}$

$3381.990 \quad 24507-54067 \quad 3.04$

$3382.404 \quad 17550-47107 \quad 2.18$

$\begin{array}{lll}3383.387 & 21039-50587 & 2.61\end{array}$

$3383.692 \quad 17727-47272 \quad 2.20$

$3383.981 \quad 17550-47093 \quad 2.18$

$3384.765 \quad 7986-37521 \quad 0.99$

$\begin{array}{lll}3387.410 & 22249-51762 & 2.76\end{array}$

$\begin{array}{lll}3388.966 & 24772-54271 & 3.07\end{array}$

$3389.748 \quad 17927-47420 \quad 2.22$

$3392.014 \quad 24336-53808 \quad 3.02$

$3392.306 \quad 17727-47197 \quad 2.20$

$3392.654 \quad 17550-47017 \quad 2.18$

$3393.382 \quad 23052-52512 \quad 2.86$

$3393.623 \quad 22838-52297 \quad 2.83$

$3393.915 \quad 18378-47834 \quad 2.28$

$\begin{array}{lll}3394.085 & 19788-49243 & 2.45\end{array}$

$3394.585 \quad 17727-47177 \quad 2.20$

$3395.080 \quad 24339-53785 \quad 3.02$

$3396.386 \quad 7728-37163 \quad 0.96$

$\begin{array}{lll}3396.977 & 7728-37158 & 0.96\end{array}$

$3397.221 \quad 24336-53763 \quad 3.02$

$3397.560 \quad 24339-53763 \quad 3.02$

$\begin{array}{lll}3397.640 & 7986-37410 & 0.99\end{array}$

$3398.220 \quad 22249-51668 \quad 2.76$

$3399.230 \quad 21999-51409 \quad 2.73$

$3399.336-17727-47136 \quad 2.20$

$\begin{array}{lll}3400.662 & 24336-53734 & 3.02\end{array}$

$3401.007 \quad 24339-53734 \quad 3.02$

$\begin{array}{lll}3401.520 & 7377-36767 & 0.91\end{array}$

$3402.256 \quad 26106-55490 \quad 3.24$

$3403.299 \quad 21999-51374 \quad 2.73$

$\begin{array}{lll}3404.356 & 17727-47093 & 2.20\end{array}$

$3404.755 \quad 21999-51361 \quad 2.73$

$3404.923 \quad 21716-51077 \quad 2.69$

$\begin{array}{lll}3405.830 & 21716-51069 & 2.69\end{array}$

$3406.442 \quad 26406-55754 \quad 3.27$

$\begin{array}{lll}3406.802 & 17927-47272 & 2.22\end{array}$

$3407.461 \quad 17550-46889 \quad 2.18$

$3409.218 \quad 26106-55430 \quad 3.24$

$\begin{array}{lll}3409.605 & 19788-49109 & 2.45\end{array}$

$3410.031 \quad 24575-53892 \quad 3.05$

$3410.171 \quad 27543-56859 \quad 3.41$

$\begin{array}{lll}3410.905 & 7377-36686 & 0.91\end{array}$

$\begin{array}{lll}3411.134 & 21716-51023 & 2.69\end{array}$

$3411.353 \quad 21999-51305 \quad 2.73$

$\begin{array}{lll}3413.134 & 17727-47017 & 2.20\end{array}$

$\begin{array}{lll}3414.564 & 26628-55906 & 3.30\end{array}$

$\begin{array}{lll}3415.532 & 17927-47197 & 2.22\end{array}$

$3416.679 \quad 20038-49298 \quad 2.48$
Mult. Arc Spk $\mathrm{CW}(\mathrm{N})$ HC R(N) KOC MS Best No. - $\log g f-\log g f \lambda \quad g A / \lambda$

$\begin{array}{rrrrrrrrr}304 & 0.62 & 0.53 & 0.51^{2} & 0.48 & 0.53 & 4.06 & 5.77 \\ 304 & 0.72 & 0.71 & 0.69^{2} & 0.93 & 0.75 & 4.28 & 5.99 \\ 83 & -0.66 & -0.74 & -0.82^{1} & -0.74 & & -0.74 & 2.79 & 4.50 \\ 447 & -1.73 & & & & -1.73 & 1.80 & 3.51 \\ 303 & -1.14 & -0.74 & & -0.66 & -0.85 & 2.68 & 4.39 \\ & & & & & & -1.07 & 2.46 & 4.17 \\ 89 & & -1.07 & & & & -1.93 & 1.60 & 3.31 \\ & -1.93 & & & & & 0.18 & 3.71 & 5.42 \\ 301 & 0.17 & 0.13 & & 0.24 & & -0.18 & 3.35 & 5.06 \\ 85 & -0.15 & -0.27 & & -0.10 & -0.20^{1} & 0.36 & 3.89 & 5.60 \\ 304 & 0.36 & 0.41 & 0.33^{1} & 0.36 & & & & \\ & & & & & & -0.62 & 2.91 & 4.62 \\ 376 & -0.75 & -0.50 & 0.40^{1} & 0.37 & & -1.44 & 2.09 & 3.80 \\ & -1.44 & & & & & -0.75 & 2.78 & 4.49 \\ 84 & -0.75 & -0.77 & -0.74^{1} & -0.74 & & -1.84 & 1.69 & 3.40 \\ 245 & -1.84 & & & & -0.27 & 3.26 & 4.97\end{array}$

$\begin{array}{lllll}83 & 0.10 & 0.01 & 0.17^{2} & 0.1\end{array}$

0.12

3.65

$-3.20 \quad 0.33$

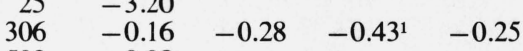

$-0.28$

3.25

$-0.82$

2.61

$\begin{array}{rrrrr}87 & -0.77 & -0.69 & -1.01^{1} & -0.83\end{array}$

2.71

$-0.13 \quad 3.40$

$0.04 \quad 3.57$

$0.43 \quad 3.96$

$-0.64 \quad 2.89$

$-1.28 \quad 2.25$

5.36

2.04

4.95

4.31

4.41

499
83

85

376
376

$\begin{array}{rr}0.42 & 0.37 \\ -0.75 & -0.63\end{array}$

$0.46^{2}-0.06$

$0.54^{1}$

5.10

5.27

5.66

4.59

$\begin{array}{lll}-1.14 & 2.39 & 4.09\end{array}$

$136-1.18$

$188-0.93$

$-1.10$

$-1.06$

$-1.00$

2.39
2.53

$-0.16 \quad 3.37$

$-1.70$

3.37
1.83

$\begin{array}{ll}-2.99 & 0.54\end{array}$

4.23

5.07

$\begin{array}{ll}25 & -2.99\end{array}$

26

503

447

26
304

$-0.83$

$\begin{array}{lll}-1.70 & -1.63^{1} & -1.62\end{array}$

$-1.65$

1.88

$-0.83$

2.70

$-0.90$

$-0.90$

$-1.90$

2.63

$\begin{array}{llll}-1.92 & -1.93 & -1.90^{1} & -1.87 \\ -0.96 & -0.60 & & -1.00\end{array}$

302

85
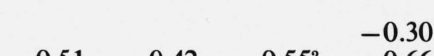

0.51
-1.06

$0.55^{2}$

0.66

$0.61^{1}$

$-1.32$

26

$-1.45$

$-1.35^{1}-1.24$

$-0.85$

1.63

$-0.30 \quad 3.23$

$0.55 \quad 4.08$

$\begin{array}{ll}-1.06 & 2.47\end{array}$

$-1.32$

$-1.34$

2.21
2.19

0.53

$-0.47$

4.06

$\begin{array}{ll}0.43^{1} & 0.44\end{array}$

$-0.44$

0.36
0.32

0.46

$-0.45$

0.42

$-0.98$

$-1.23$

3.06

3.95

2.55

2.30

$-0.61 \quad 2.92$

0.22

0.13

3.75

$\begin{array}{ll}0.091 & 0.19 \\ 0.77^{2} & 0.24\end{array}$

676
85

$\begin{array}{lr}0.64 & -0.58 \\ 0.23 & 0.23\end{array}$

$0.09 \quad 0.03$

$0.21^{1}$

0.86

3.66

4.39

$-0.40 \quad 3.13$

$-1.87 \quad 1.66$

$-0.57$

1.66
2.96

$0.36 \quad 3.89$

$-2.14$

3.89
1.39

$-0.96$

2.57

0.01

3.54

0.68

3.54
4.21

0.68
$-1.00 \quad 2.53$

$-0.29 \quad 3.24$

$-1.82$ 
TABLE 3.-Oscillator Strengths for Ultraviolet Lines of Fe I-Continued

Wave-

length Energy Levels e.p.

K

Mult. Arc Spk $\mathrm{CW}(\mathrm{N}) \quad \mathrm{HC} \quad \mathrm{R}(\mathrm{N})$ KOC $\quad$ MS Best

$\log$ gf $\lambda \quad \begin{array}{ll}\log \\ \mathrm{gA} / \lambda\end{array}$

$\begin{array}{lll}3417.273 & 8155-37410 & 1.0\end{array}$

$3417.843 \quad 17927-47177 \quad 2.22$

$3418.176 \quad 26479-55727 \quad 3.28$

$\begin{array}{lll}3418.507 & 17927-47172 & 2.22 \\ 3418.905 & 24507-53748 & 3.04\end{array}$

$3419.154 \quad 26140-55379 \quad 3.24$

$3419.706 \quad 22947-52181 \quad 2.84$

$\begin{array}{lll}3422.499 & 24119-53329 & 2.99\end{array}$

$3422.658 \quad 17927-47136 \quad 2.22$

$3424.286 \quad 17550-46745 \quad 2.18$

$3425.009 \quad 24575-53763 \quad 3.05$

$\begin{array}{lll}3426.337 & 18378-47556 & 2.28\end{array}$

$3426.383 \quad 17550-46727 \quad 2.18$

$3426.637 \quad 17727-46902 \quad 2.20$

$3427.121 \quad 17550-46721 \quad 2.18$

$3428.195 \quad 17727-46889 \quad 2.20$

$3428.746 \quad 29056-58213 \quad 3.60$

$3431.815 \quad 22838-51969 \quad 2.83$

$3432.023 \quad 23052-52181 \quad 2.86$

$3434.029 \quad 22249-51361 \quad 2.76$

$\begin{array}{lll}3436.045 & 26351-55446 & 3.27\end{array}$

$3437.046 \quad 24575-53661 \quad 3.05$

$3437.631 \quad 19621-48703 \quad 2.43$

$3437.952 \quad 26351-55430 \quad 3.27$

$3439.039 \quad 21999-51069 \quad 2.73$

$\begin{array}{lll}3440.607 & 0-29056 & 0.00\end{array}$

$\begin{array}{lll}3440.990 & 416-29469 & 0.05\end{array}$

$\begin{array}{lll}3442.364 & 18378-47420 & 2.28\end{array}$

$3442.671 \quad 7728-36767 \quad 0.96$

$3442.979 \quad 24772-53808 \quad 3.07$

$\begin{array}{lll}3443.878 & 704-29733 & 0.09\end{array}$

$3445.151 \quad 17727-46745 \quad 2.20$

$3446.791 \quad 21039-50043 \quad 2.61$

$3446.947 \quad 8155-37158 \quad 1.01$

$\begin{array}{lll}3447.280 & 17727-46727 & 2.20\end{array}$

3448.786

$22838-51826 \quad 2.83$

$20641-49628 \quad 2.56$

3450.330

3451.628

3451.917

$17927-46902 \quad 2.22$

$19552-48516 \quad 2.42$

$17927-46889 \quad 2.22$

$3452.276 \quad 7728-36686 \quad 0.96$

$3453.022 \quad 22249-51201 \quad 2.76$

$3457.090 \quad 29056-57974 \quad 3.60$

$\begin{array}{lll}3457.512 & 19788-48703 & 2.45\end{array}$

$3458.304 \quad 19552-48460 \quad 2.42$

$3459.429 \quad 21716-50614 \quad 2.69$

$3459.911 \quad 24336-53230 \quad 3.02$

$3462.354 \quad 17727-46601 \quad 2.20$

3462.808

3463.304

$22838-51708 \quad 2.83$

$11976-40842 \quad 1.48$

3464.914

$20875-49727 \quad 2.59$

$888-29733 \quad 0.11$

$\begin{array}{rrr}3466.279 & 19390-48231 & 2.40 \\ 3466.500 & 6928-35768 & 0.86 \\ 3468.847 & 20641-49461 & 2.56\end{array}$

$\begin{array}{rrr}3466.279 & 19390-48231 & 2.40 \\ 3466.500 & 6928-35768 & 0.86 \\ 3468.847 & 20641-49461 & 2.56\end{array}$

3469.012

$26628-55446 \quad 3.30$

$3469.390 \quad 22947-51762 \quad 2.84$

$3469.834 \quad 21039-49851 \quad 2.61$

$\begin{array}{lll}3471.267 & 17927-46727 & 2.22\end{array}$

3471.346

$\begin{array}{ll}17927-46727 & 2.22 \\ 18378-47177 & 2.28\end{array}$

$$
\text { No. }
$$

$26-2.14$

$81 \quad 0.44$

$577-0.51$

$\begin{array}{ll}81 & 0.37\end{array}$

$-0.94$

0.39

$0.45^{2}$

1.17

$0.48^{1}$

$\begin{array}{llll}0.29 & 0.40^{2} & 0.62 & 0.40^{1}\end{array}$

$\begin{array}{rr}576 & -0.65 \\ 377 & -0.46 \\ 444 & 0.30 \\ 85 & 0.23 \\ 81 & 0.41\end{array}$

541

135

82

82

81

81

836

376

377

300

$614-0.88$

$\begin{array}{rr}639 & -0.88 \\ 187 & -1.63\end{array}$

$187-1.63$

$614-0.09$

299

6
6
134
26
499

26
499

8

84
24

244

26
82

$-0.73$

$\begin{array}{rrrrr} & -0.52 & -0.30^{1} & -0.11 & -0.49^{1} \\ -0.19 & -0.69 & -0.93^{2} & -0.75 & -0.79^{1} \\ -1.64 & -0.23 & -0.13^{1} & -0.60 & \\ & -1.89 & & -1.64 & \\ & & & -0.50 & \end{array}$

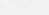

0.52

$-0.96$

$-0.50$

$-1.08$

-0.96
0.45

$-1.21$

$-0.9$

$-1.19^{1}$

$0.35^{1}$
$0.43^{1}$

$\begin{array}{ll}0.41^{2} & 0.54\end{array}$

$\begin{array}{ll}0.18^{2} & -0.19 \\ 0.09 & -0.09\end{array}$

$\begin{array}{lr}0.18^{2} & -0.09 \\ 0.13^{1} & 0.24\end{array}$

$0.05^{1}$

$0.05^{1}$

$0.98^{1}$

$0.34^{1}$

1.13

1.82

-2.53
0.06

-0.44
$-0.05 \quad-0.97$

$372-1.36$

242

$-1.25$

0.14

$-0.45$

$-1.02$

$0.04^{1}$

0.02
-0.54

$\begin{array}{rr}0.14^{2} & 0.27 \\ & -0.54\end{array}$

$0.14^{1}$

81

25

301

835

187

139

$\begin{array}{llll}-1.08 & -1.34 & -1.06^{1} & -1.07 \\ -0.05 & -0.22 & -0.29^{1} & -0.06\end{array}$

0.01

$\begin{array}{rr}0.20^{1} & -0.54 \\ & 0.25\end{array}$

$0.14^{1}$

$-0.20$

$-1.58$

$\begin{array}{lll}-0.22 & -0.29^{1} & -0.06\end{array}$

$-0.32^{1}$

$-0.17$

$\begin{array}{lll}-0.29 & -0.31^{1} & -0.23\end{array}$

$\begin{array}{rrrrr}297 & -0.41 & -0.53 & & 0.19\end{array}$

501

79

373
48

0.28

$-1.34$

$\begin{array}{rr}0.18^{1} & 0.22 \\ -1.18^{1} & -0.88\end{array}$

$-1.65-1.66$

$\begin{array}{rrrrrr}241 & -1.00 & -0.92 & & -0.98 & \\ 6 & & -0.84 & -1.03^{1} & -0.87 & -1.00^{1} \\ 185 & & & & -1.36 & \\ 24 & -1.86 & -1.94 & & & \end{array}$

$\begin{array}{lllll}242 & -0.12 & -0.11 & -0.15^{1} & -0.16\end{array}$

$\begin{array}{rrrrrr}614 & 0.08 & 0.13 & & & \\ 375 & -1.32 & & & -0.01 & \\ 242 & -0.32 & -0.44 & -0.50^{1} & -0.26 & \\ 82 & & & & -0.28 & -0.21^{1} \\ 130 & & & & -0.09 & \end{array}$ $\begin{array}{rrr}-2.14 & 1.39 & 3.08 \\ -0.44 & 3.97 & 5.66\end{array}$

5.66

$\begin{array}{lll}0.41 & 3.94 & 5.63\end{array}$

$\begin{array}{lll}-0.94 & 2.59 & 4.28\end{array}$

$\begin{array}{lll}-0.56 & 2.97 \quad 4.66\end{array}$

$\begin{array}{lll}-0.52 & 3.01 \quad 4.70\end{array}$

$\begin{array}{lll}0.18 & 3.71 & 5.40\end{array}$

$\begin{array}{lll}0.27 & 3.80 & 5.49\end{array}$

$\begin{array}{lll}0.42 & 3.95 & 5.64\end{array}$

$\begin{array}{lll}0.42 & 3.95 & 5.64\end{array}$

$\begin{array}{lll}-0.19 & 3.34 & 5.03\end{array}$

$\begin{array}{lll}0.09 & 3.62 & 5.31\end{array}$

$\begin{array}{lll}0.13 & 3.66 & 5.35\end{array}$

$\begin{array}{lll}0.87 & 4.40 & 6.09\end{array}$

$\begin{array}{lll}0.30 & 3.84 & 5.52\end{array}$

$\begin{array}{lll}-0.14 & 3.40 & 5.08\end{array}$

$\begin{array}{lll}-0.14 & 3.40 & 5.08\end{array}$

$\begin{array}{lll}-1.10 & 2.44 & 4.12\end{array}$

$\begin{array}{lll}-0.96 & 2.58 & 4.26\end{array}$

$\begin{array}{lll}-0.88 & 2.66 & 4.34\end{array}$

$\begin{array}{lll}0.07 & 3.61 & 5.29\end{array}$

$\begin{array}{lll}-1.44 & 2.10 & 3.78\end{array}$

$\begin{array}{lll}-0.02 & 3.52 & 5.20\end{array}$

$\begin{array}{lll}-0.68 & 2.86 & 4.53\end{array}$

$\begin{array}{lll}-0.44 & 3.10 & 4.77\end{array}$

$\begin{array}{lll}-0.82 & 2.72 & 4.39\end{array}$

$\begin{array}{lll}-0.18 & 3.36 & 5.03\end{array}$

$\begin{array}{lll}-1.72 & 1.82 & 3.49\end{array}$

$\begin{array}{lll}-0.50 & 3.04 & 4.71\end{array}$

$\begin{array}{lll}-1.09 & 2.45 & 4.12\end{array}$

$\begin{array}{lll}0.57 & 4.11 & 5.78\end{array}$

$\begin{array}{lll}-1.08 & 2.46 & 4.13\end{array}$

$\begin{array}{lll}-2.53 & 1.01 & 2.68\end{array}$

\begin{tabular}{l}
$0.02-3.56-5.23$ \\
\hline
\end{tabular}

$\begin{array}{lll}-1.19 & 2.35 & 4.02\end{array}$

$\begin{array}{lll}-1.25 & 2.29 & 3.96\end{array}$

$\begin{array}{lll}0.14 & 3.68 & 5.35\end{array}$

$\begin{array}{lll}-0.51 & 3.03 & 4.70\end{array}$

$\begin{array}{lll}0.15 & 3.69 & 5.36\end{array}$

$\begin{array}{lll}-1.14 & 2.40 \quad 4.07\end{array}$

$\begin{array}{lll}-0.16 & 3.38 & 5.05\end{array}$

$\begin{array}{lll}-0.26 & 3.28 & 4.95\end{array}$

$\begin{array}{lll}-1.58 & 1.96 & 3.63\end{array}$

$\begin{array}{lll}-0.25 & 3.29 & 4.96\end{array}$

$\begin{array}{lll}-0.47 & 3.07 & 4.74\end{array}$

$\begin{array}{lll}0.21 & 3.75 & 5.42\end{array}$

$\begin{array}{lll}-1.00 & 2.54 & 4.21\end{array}$

$\begin{array}{lll}-1.34 & 2.20 & 3.87\end{array}$

$\begin{array}{lll}-1.66 & 1.88 & 3.55\end{array}$

$\begin{array}{lll}-0.97 & 2.57 & 4.23\end{array}$

$\begin{array}{lll}-0.94 & 2.60 & 4.26\end{array}$

$\begin{array}{lll}-1.36 & 2.18 & 3.84\end{array}$

$\begin{array}{lll}-1.90 & 1.64 \quad 3.30\end{array}$

$\begin{array}{lll}-0.14 & 3.40 & 5.06\end{array}$

$\begin{array}{lll}0.10 & 3.64 & 5.30\end{array}$

$\begin{array}{lll}-1.32 & 2.22 & 3.88\end{array}$

$\begin{array}{lll}-0.38 & 3.16 & 4.82\end{array}$

$\begin{array}{lll}-0.24 & 3.30 & 4.96\end{array}$ 
TABLE 3.-Oscillator Strengths for Ultraviolet Lines of Fe I-Continued

Wave-

length Energy Levels e.p.

A

3473.303

3473.497

3475.451

3475.651

3475.867

3476.336

3476.704

3476.853

3477.007

3477.856

3478.374

3478.788

3479.683

3481.558

3483.009

3484.858

3484.972

3485.342

3486.556

3489.670

\section{4}

$21999-50614 \quad 2.73$

$3494.170 \quad 19552-48163 \quad 2.42$

$3495.288 \quad 20641-49243 \quad 2.56$

$3496.190 \quad 19788-48383 \quad 2.45$

3497.110

3497.842

3500.164

3500.568

3504.455

3504.864

3505.065

3506.500

3507.139

3507.390

3508.494

3509.120

3509.736

3509.870

3510.446

3511.748

3512.080

3512.239

3512.970

3513.065

3513.820

3514.626

3516.403

3516.550

3518.680

3518.860

3520.855

3521.263

3521.833

3522.268

3522.896

3523.300

3524.075

3524.242
$416-29056 \quad 0.05$

3.05

$704-29469 \quad 0.09$

$17550-46314 \quad 2.18$

$18378-47136 \quad 2.28$

$19552-48305 \quad 2.42$

$19621-48362 \quad 2.43$

$\begin{array}{ll}19552-48290 & 2.42 \\ 23784-52514 & 2.95\end{array}$

$18378-47093 \quad 2.28$

$19788-48476 \quad 2.45$

$17727-46410 \quad 2.42$

$17927-46601 \quad 2.22$

$23784-52431 \quad 2.95$

$17550-46137 \quad 2.18$

$888-29469 \quad 0.11$

$23193-51755 \quad 2.87$

$20875-49433 \quad 2.59$

$22838-51365 \quad 2.83$

$18378-46902 \quad 2.28$

$24336-52858 \quad 3.02$

$18378-46889 \quad 2.28$

$29469-57974 \quad 3.65$

$24772-53275 \quad 3.07$

$24119-52613 \quad 2.99$

$22846-51335 \quad 2.83$

$23270-51755 \quad 2.88$

$17927-46410 \quad 2.22$

$20038-48516 \quad 2.48$

$20641-49109 \quad 2.56$

$22997-51462 \quad 2.85$

$22997-51461 \quad 2.85$

$24772-53230 \quad 3.07$

$12561-41018 \quad 1.56$

$6928-35379 \quad 0.86$

$19390-47835 \quad 2.40$

$24339-52769 \quad 3.02$

$23111-51540 \quad 2.86$

$23193-51604 \quad 2.87$

$17727-46137 \quad 2.20$

$21039-49433 \quad 2.61$

$7377-35768 \quad 0.91$

$17927-46314 \quad 2.22$

$22846-51229 \quad 2.83$

$23193-51570 \quad 2.87$

$23193-51567 \quad 2.87$

$20875-49243 \quad 2.59$

$18378-46745 \quad 2.28$ $\begin{array}{rr}978-29733 & 0.12 \\ 20875-49628 & 2.59\end{array}$
Mult.

No.

$\begin{array}{lllllll}\text { Arc } & \text { Spk } & \mathrm{CW}(\mathrm{N}) & \mathrm{HC} & \mathrm{R}(\mathrm{N}) & \mathrm{KOC} & \mathrm{MS}\end{array}$

$-0.31$

$\begin{array}{rrrrrr}26 & -2.49 & & & -1.10 & \\ 6 & & -0.74 & -0.93^{1} & -0.80 & -0.89^{1} \\ 78 & 0.28 & -0.09 & & 0.39 & \\ 186 & -0.57 & & & -0.20 & \\ & & & & & \\ 133 & -0.86 & -0.78 & & -0.50 & \\ 6 & & -1.07 & -1.36^{2} & -1.19 & -1.22^{2} \\ 242 & & -0.08 & & -0.13 & \\ 139 & & -0.29 & & -0.54 & \\ 82 & -0.68 & -0.83 & -0.97^{1} & -0.84 & -0.78^{1} \\ 185 & -1.45 & & & & \\ 137 & -1.24 & & & -1.30 & \\ 443 & -1.04 & & & -0.70 & \\ 132 & -1.60 & & & 0.86 & \\ 24 & -1.72 & -2.01 & -2.12^{1} & -1.13 & \\ & & & & & \end{array}$

$185-1.19$

$\begin{array}{ll}185 & -1.19 \\ 138 & -0.78\end{array}$

$\begin{array}{rr}78 & -0.02 \\ 79 & -1.15\end{array}$

-0.79
-0.15
-1.08
0.18

$$
-2.12^{1}
$$

$-0.17$

-0.17
-0.76

$\begin{array}{rr}-0.01^{2} & -0.76 \\ -0.05\end{array}$

$0.121 \quad-1.13$

0.15

$\begin{array}{lll}-1.04^{2} & -0.47 & -0.93^{1}\end{array}$

$-1.72$

$-1.37$

$-0.40$

$-1.10 \quad-0.86$

$-0.46$

$\begin{array}{rr}0.24^{2} & -0.29\end{array}$

$-1.03$

0.27

0.20

$-0.94$

0.12

$0.20^{2}$

0.12
-1.29

$-1.32^{4}$

$-1.23-1.45^{1}$

$-0.66$

$-0.16$

$-0.33$

$-0.81^{1}$

$-0.47$

$-1.09$

-0.57
0.03

$-0.73$

$-0.38$

$-0.11$

$-0.13$

$\begin{array}{rr} & -0.47 \\ & -0.58 \\ & 0.07 \\ 0.09^{1} & 0.08\end{array}$

835

500

442

326

327

78

139

238

327

326

501

48

24
183

442
326

327

78

238

24

78

326

330

326

239

130

$\begin{array}{rrrr}0.32 & 0.18 & -0.21^{1} & -0.38 \\ -0.77 & & & -0.74 \\ -0.78 & & & \\ -0.66 & -0.63 & -1.29^{1} & -0.80 \\ & & & \\ -0.86 & -0.39 & -0.85^{1} & -0.40 \\ & & & -0.92 \\ -0.49 & & & -0.92 \\ \end{array}$

$-0.63$

$-1.41-1.41$

$-0.77$

0.08

$-0.20$

$-0.39$

-0.68
-0.52

$-0.32$

$-0.35 \quad-0.58$

$-0.50^{1} \quad-0.30$

$-0.40^{1} \quad-0.18$

$-0.23$

$-0.31$

$-1.48^{1} \quad-0.87$
-0.76

$\begin{array}{lll} & -0.76 & \\ -0.49^{1} & -0.34 & -0.46^{1}\end{array}$

$-0.10$

0.00

$-0.99$

$-0.57-0.71$

$-0.22-0.55$

$0.05-0.01$

$0.01-0.21$
$-0.39$

0.08
$-1.38$

$-1.24$

$-1.04$

$-1.60$

$-1.97$

$-1.19$

$-0.78$

$-0.05$

$-1.12$

0.18

$-1.00$

$-1.54$

$-0.40$

-0.98
0.25

$-1.03$

0.18

$-1.27 \quad-1.27$

$-1.45$

$-1.09$

$-0.63$

$-0.38$

$-0.76$

$\log$ gf $\lambda$

$3.23 \quad 4.89$

$\begin{array}{lll}-2.49 & 1.05 & 2.71\end{array}$

$\begin{array}{lll}-0.84 & 2.70 & 4.36\end{array}$

$\begin{array}{rrr}0.10 & 3.64 & 5.30\end{array}$

4.82

4.49

3.96

5.10

4.78

4.38

3.82

2.30

2.50

1.94

3.96

4.16

3.60

3.23

2.35

4.01

$\begin{array}{ll}2.76 & 4.42\end{array}$

$\begin{array}{ll}3.42 & 4.08\end{array}$

3.72

5.38

2.54

4.20

2.00

$\begin{array}{ll}3.14 & 4.79\end{array}$

2.56
3.79

4.21
5.44

\subsection{1}

3.72

2.09

4.16

5.37

3.92

3.74

2.45

2.91

4.10

$0.00 \quad 3.54$

$0.00 \quad 3.54$

$-0.44 \quad 3.10$

4.56

5.19

5.19

3.16

4.81

2.79

2.77

4.43 
TABLE 3.-Oscillator Strengths for Ultraviolet Lines of Fe I-Continued

Wave- Low

Mult. Arc Spk $\mathrm{CW}(\mathrm{N})$ HC $\mathrm{R}(\mathrm{N})$ KOC MS Best

$\log$

No. $\quad \log g f-\log g f \lambda \quad \mathrm{gA} / \lambda$

\begin{tabular}{|c|c|c|c|c|c|c|c|c|c|c|c|c|}
\hline 3525.856 & $22997-51351$ & 2.85 & 329 & -0.33 & & & -0.14 & & & -0.24 & 3.31 & 4.94 \\
\hline 3526.042 & $704-29056$ & 0.09 & 6 & -1.36 & & $-1.61^{1}$ & -1.19 & $-1.59^{1}$ & & -1.52 & 2.03 & 3.66 \\
\hline 3526.168 & $7728-36079$ & 0.96 & 24 & & & $-0.89^{1}$ & -0.67 & & & -0.78 & 2.77 & 4.40 \\
\hline 3526.230 & $23111-51462$ & 2.86 & 327 & & & & 0.67 & & & 0.67 & 4.22 & 5.85 \\
\hline 3526.377 & $23111-51461$ & 2.86 & 326 & 0.72 & 0.60 & & & & & 0.66 & 4.21 & 5.84 \\
\hline 3526.470 & $18378-46727$ & 2.28 & 131 & 0.25 & & & & & & 0.25 & 3.80 & 5.43 \\
\hline 3526.673 & $23193-51540$ & 2.87 & 326 & 0.70 & 0.38 & & 0.74 & & & 0.61 & 4.16 & 5.79 \\
\hline 3527.792 & $22997-51335$ & 2.85 & 326 & 0.48 & 0.13 & $0.46^{1}$ & 0.59 & & & 0.36 & 3.91 & 5.54 \\
\hline 3528.316 & $12561-40895$ & 1.56 & & -2.25 & & & & & & -2.25 & 1.30 & 2.93 \\
\hline 3528.942 & $6928-35257$ & 0.86 & 23 & -3.73 & & & & & & -3.73 & -0.18 & 1.45 \\
\hline 3529.531 & $24575-52899$ & 3.05 & 537 & -0.69 & & & -0.55 & & & -0.62 & 2.93 & 4.56 \\
\hline 3529.818 & $23245-51567$ & 2.88 & 326 & 0.56 & 0.24 & $0.44^{1}$ & 0.44 & & & 0.42 & 3.97 & 5.60 \\
\hline 3530.385 & $22650-50968$ & 2.81 & 326 & 0.12 & & $0.10^{1}$ & 0.17 & & & 0.13 & 3.68 & 5.31 \\
\hline 3531.446 & $19621-47930$ & 2.43 & 182 & -0.99 & -0.92 & & -1.12 & & & -1.01 & 2.54 & 4.17 \\
\hline 3533.008 & $23270-51567$ & 2.88 & 326 & 0.60 & 0.42 & & 0.50 & & & 0.51 & 4.06 & 5.69 \\
\hline 3533.201 & $23245-51540$ & 2.88 & 326 & 0.90 & 0.58 & $0.63^{1}$ & 0.93 & & & 0.76 & 4.31 & 5.94 \\
\hline 3534.530 & $28820-57104$ & 3.57 & 811 & -0.44 & -0.36 & & -0.18 & & & -0.33 & 3.22 & 4.85 \\
\hline 3534.914 & $12561-40842$ & 1.56 & 48 & -1.69 & -1.67 & & & & & -1.68 & 1.87 & 3.50 \\
\hline 3536.556 & $23193-51461$ & 2.87 & 326 & 0.99 & 0.73 & $1.00^{2}$ & 1.21 & & & 0.99 & 4.54 & 6.17 \\
\hline 3537.491 & $20875-49135$ & 2.59 & 239 & -0.44 & -0.46 & & -0.40 & & & -0.43 & 3.12 & 4.75 \\
\hline 3537.729 & $21039-49298$ & 2.61 & 239 & -0.04 & -0.03 & & 0.11 & & & 0.01 & 3.56 & 5.19 \\
\hline 3537.896 & $22846-51103$ & 2.83 & 327 & 0.25 & -0.11 & & 0.39 & & & 0.07 & 3.62 & 5.25 \\
\hline 3538.310 & $28605-56859$ & 3.55 & 775 & -0.60 & -0.52 & & -0.30 & & & -0.47 & 3.08 & 4.71 \\
\hline 3538.550 & $20038-48290$ & 2.48 & 137 & -1.14 & -0.91 & & & & & -1.02 & 2.53 & 4.16 \\
\hline 3538.790 & $28820-57070$ & 3.57 & 811 & -0.89 & & & -0.03 & & & -0.89 & 2.66 & 4.29 \\
\hline 3540.121 & $23111-51351$ & 2.86 & 329 & 0.25 & -0.06 & $0.19^{1}$ & 0.45 & & & 0.13 & 3.68 & 5.31 \\
\hline 3540.711 & $7377-35612$ & 0.91 & 23 & -1.71 & -1.68 & & -1.47 & & & -1.62 & 1.93 & 3.56 \\
\hline 3541.083 & $22997-51229$ & 2.85 & 326 & 1.15 & 0.79 & $1.08^{2}$ & 1.10 & & & 1.04 & 4.59 & 6.22 \\
\hline 3542.076 & $23111-51335$ & 2.86 & 326 & 1.15 & & $1.01^{2}$ & 1.16 & & & 1.01 & 4.56 & 6.19 \\
\hline 3542.243 & $18378-46601$ & 2.28 & 128 & & & & -0.33 & & & -0.33 & 3.22 & 4.85 \\
\hline 3543.392 & $19621-47835$ & 2.43 & 183 & -0.93 & -0.82 & & -0.85 & & & -0.87 & 2.68 & 4.31 \\
\hline 3543.669 & $27543-55754$ & 3.41 & 734 & 0.21 & 0.21 & $0.12^{1}$ & 0.28 & & & 0.20 & 3.75 & 5.38 \\
\hline 3544.631 & $21039-49243$ & 2.61 & 239 & -0.49 & -0.59 & $-0.68^{1}$ & -0.45 & & & -0.55 & 3.00 & 4.63 \\
\hline 3545.639 & $22997-51192$ & 2.85 & 321 & 0.35 & 0.24 & $0.33^{1}$ & 0,50 & $0.59^{1}$ & & 0.40 & 3.95 & 5.57 \\
\hline 3545.832 & $24575-52769$ & 3.05 & 536 & -0.27 & 0.08 & & -0.56 & & & -0.10 & 3.45 & 5.07 \\
\hline 3546.210 & $19621-47812$ & 2.43 & 183 & -1.39 & & & -0.99 & & & -1.19 & 2.36 & 3.98 \\
\hline 3547.203 & $22650-50833$ & 2.81 & 321 & -0.29 & -0.24 & $-0.37^{1}$ & -0.31 & $-0.16^{1}$ & & -0.27 & 3.28 & 4.90 \\
\hline 3548.037 & $24336-52512$ & 3.02 & 496 & -0.31 & -0.27 & $-0.40^{1}$ & -0.19 & & & -0.29 & 3.26 & 4.88 \\
\hline 3549.868 & $12969-41131$ & 1.61 & 48 & -1.30 & -1.44 & $-1.43^{1}$ & -1.43 & & & -1.40 & 2.15 & 3.77 \\
\hline 3551.114 & $22997-51149$ & 2.85 & 321 & -1.16 & & & & & & -1.16 & 2.39 & 4.01 \\
\hline 3552.112 & $24772-52916$ & 3.07 & 499 & -0.30 & -0.17 & & -0.12 & & & -0.20 & 3.35 & 4.97 \\
\hline 3552.420 & $19788-47930$ & 2.45 & 182 & -1.45 & & & -0.97 & & & -1.45 & 2.10 & 3.72 \\
\hline 3552.828 & $23193-51331$ & 2.87 & 321 & 0.20 & -0.08 & $0.10^{1}$ & 0.17 & $0.36^{1}$ & & 0.15 & 3.70 & 5.32 \\
\hline 3553.741 & $28820-56951$ & 3.57 & 810 & 0.97 & 1.06 & $0.78^{1}$ & 1.00 & & & 0.95 & 4.50 & 6.12 \\
\hline 3554.120 & $7728-35856$ & 0.96 & 23 & -1.40 & -1.60 & & -1.82 & & & -1.50 & 2.05 & 3.67 \\
\hline 3554.500 & $23245-51370$ & 2.88 & 325 & -0.10 & 0.04 & & 0.11 & & & 0.02 & 3.57 & 5.19 \\
\hline 3554.922 & $22846-50968$ & 2.83 & 326 & 1.47 & 1.13 & $1.19^{2}$ & 1.11 & & & 1.22 & 4.77 & 6.39 \\
\hline 3556.680 & $23111-51219$ & 2.86 & 325 & -0.24 & & & -0.38 & & & -0.31 & 3.24 & 4.86 \\
\hline 3556.877 & $22997-51103$ & 2.85 & 327 & 0.92 & 0.53 & $0.85^{1}$ & 0.99 & & & 0.82 & 4.37 & 5.99 \\
\hline 3558.517 & $7986-36079$ & 0.99 & 24 & -0.30 & -0.04 & $-0.27^{2}$ & -0.26 & $-0.39^{3}$ & -0.24 & -0.28 & 3.27 & 4.89 \\
\hline 3559.506 & $24772-52858$ & 3.07 & 498 & 0.12 & 0.30 & $-0.48^{1}$ & 0.21 & & & 0.21 & 3.76 & 5.38 \\
\hline 3560.076 & $23111-51192$ & 2.86 & 321 & -1.09 & & & & & & -1.09 & 2.46 & 4.08 \\
\hline 3560.705 & $26225-54301$ & 3.25 & 675 & 0.04 & 0.03 & $-0.65^{1}$ & -0.07 & & & 0.00 & 3.55 & 5.17 \\
\hline 3562.269 & $26225-54289$ & 3.25 & & -1.07 & & & & & & -1.07 & 2.48 & 4.10 \\
\hline 3564.110 & $12969-41018$ & 1.61 & 48 & -1.66 & -1.65 & & & & & -1.66 & 1.89 & 3.51 \\
\hline 3564.533 & $19788-47834$ & 2.45 & 183 & -0.67 & -0.40 & & & & & -0.54 & 3.01 & 4.63 \\
\hline 3565.381 & $7728-35768$ & 0.96 & 24 & & & $0.16^{2}$ & 0.21 & $-0.01^{3}$ & 0.26 & 0.11 & 3.66 & 5.28 \\
\hline 3565.583 & $23111-51149$ & 2.86 & 321 & 0.75 & & & 0.66 & $0.73^{1}$ & & 0.71 & 4.26 & 5.88 \\
\hline 3566.316 & $18378-46410$ & 2.28 & 127 & -0.86 & -0.90 & & & & & -0.88 & 2.67 & 4.29 \\
\hline 3566.590 & $19390-47420$ & 2.40 & 181 & & & & -1.23 & & & -1.23 & 2.32 & 3.94 \\
\hline
\end{tabular}


TABLE 3.-Oscillator Strengths for Ultraviolet Lines of Fe I-Continued

Wave-

$\begin{array}{ccc}\text { ength } & \text { Energy Levels } & \text { e.p. } \\ \AA & K & \text { Volts }\end{array}$

3567.038
3567.360

3567.360

3568.423

3568.828

3568.978

3570.100

3570.243

3571.226

3571.995

3572.600

3573.400

3573.836

3573.896

3574.256

3575.118

3575.249

3575.374

3575.976

3576.760

3578.380

3579.829

3581.195

3581.650

3581.816

3582.201

3582.560

3582.690

3583.337

3584.663

3584.790

3584.960

3585.193

3585.321

3585.707

3586.114

3586.740

3586.986

3587.240

3587.424

3587.752

3588.516

3588.615

3588.918

3589.106

3589.456

3589.586

3590.086

3590.990

3591.345

3591.485

3592.486

3592.680

3592.881

3593.329

3594.632

3595.308

3595.857

3596.198

3597.050

$\begin{array}{ll}23193-51219 & 2.87 \\ 19788-47812 & 2.45 \\ 26140-54161 & 3.24 \\ 23193-51208 & 2.87 \\ 26225-54237 & 3.25\end{array}$

$21716-49727 \quad 2.69$

$7377-35379 \quad 0.91$

$22650-50652 \quad 2.81$

$22846-50833 \quad 2.83$

$22997-50980 \quad 2.85$

$26624-54600 \quad 3.30$

$19390-47363 \quad 2,40$

$26628-54600 \quad 3.30$

$26479-54449 \quad 3.28$

$23245-51208 \quad 2.88$

$22846-50808 \quad 2.83$

$24336-52297 \quad 3.02$

$23193-51149 \quad 2.87$

$26351-54301 \quad 3.27$

$23270-51208 \quad 2.88$

$26140-54067 \quad 3.24$

$6928-34844 \quad 0.86$

$21716-49628 \quad 2.69$

$24772-52683 \quad 3.07$

$19788-47693 \quad 2.45$

$23245-51149 \quad 2.88$

$26550-54449 \quad 3.29$

$21716-49604 \quad 2.69$

$23111-50999 \quad 2.86$

$26351-54237 \quad 3.27$

$23784-51668 \quad 2.95$

$7728-35612 \quad 0.96$

$7377-35257 \quad 0.91$

$26106-53983 \quad 3.24$

$22650-50523 \quad 2.81$

$\begin{array}{ll}7986-35856 & 0.99\end{array}$

$23111-50980 \quad 2.86$

$19552-47420 \quad 2.42$

26406-54271

3.27

$23711-51570 \quad 2.94$

22846-50704 2.83

$23193-51048 \quad 2.87$

6928-34782 0.86

$21999-49851 \quad 2.73$

$24119-51969 \quad 2.99$

$23784-51630 \quad 2.95$

$25900-53739 \quad 3.21$

$22997-50833 \quad 2.85$

$26550-54386 \quad 3.29$

$20875-48703 \quad 2.59$

$26140-53967 \quad 3.24$

$17727-45552 \quad 2.20$

$26340-54161 \quad 3.26$

$22997-50808 \quad 2.85$

$23193-50999 \quad 2.87$

$19788-47590 \quad 2.45$

$19621-47420 \quad 2.43$

$26340-54132 \quad 3.26$
$11976-39970 \quad 1.48$

$26106-54014 \quad 3.24$
Mult. Arc Spk $\mathrm{CW}(\mathrm{N})$ HC $\mathrm{R}(\mathrm{N})$ KOC $\mathrm{MS}$ Best

No.

325

183

321
673

294

326

46

321

325

673

181

611

574

$\begin{array}{rrrr}-0.02 & -0.33 & -0.45^{1} & 0.14 \\ -0.88 & -1.01 & & -0.72\end{array}$

$\begin{array}{rrr}-0.45^{1} & 0.14 & \\ & -0.72 & \\ & & -0.13^{1} \\ & 0.07 & \\ -0.24^{1} & 0.01 & \\ 0.43^{2} & 0.30 & 0.35^{4} \\ & & \\ 0.66^{1} & -0.24 & \\ & 0.70 & 0.87^{1} \\ & -0.24 & \\ & 0.90 & \\ & -0.14 & \\ & 0.72 & \end{array}$

$-0.95$

0.72

$\begin{array}{lll}321 & -0.22 & -0.34\end{array}$

322

496

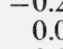

321

0.53

0.43

(n)

\begin{tabular}{lll} 
& & $0.03^{1}$ \\
$0.19^{1}$ & 0.43 & $0.18^{1}$ \\
$-0.50^{1}$ & 0.07 & \\
\hline
\end{tabular}

$321-0.30 \quad-0.66$

573
23

23

497

$-0.71$

$\begin{array}{ll}-0.26 & 0.09\end{array}$

$-0.25^{1}$

0.04

$0.16^{1}$

$0.96^{1}$

$\begin{array}{lll}0.58^{2} & 0.59 & 0.61^{3}\end{array}$

612

$\begin{array}{ll}-0.01 & -0.03\end{array}$

-0.12
0.03

\begin{tabular}{ll}
$0.57 \quad 0.73$ \\
\hline
\end{tabular}

181

328

574

$-0.74$

0.08

$-0.27$

0.90

0.05

$-1.35$

$\begin{array}{rr}0.24^{1} & -0.47 \\ 0.722^{1} & 0.02\end{array}$

$322-0.08$

$611 \quad 0.91$

$438-0.19$

23

$-0.43$

$-0.61$

0.79

$0.72^{1}$

0.83

0.79

1.07

0.35

0.83

$\begin{array}{lll}-0.30 & -0.49^{1} & -0.10\end{array}$

$\begin{array}{lll}-0.61 & -0.67^{2} & -0.57\end{array}$

61

325

1.10

1.05

$1.00^{1} \quad 1.12$

-0.22
-0.40

$-0.22$

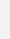

$-0.41^{1} \quad-0.37$
-0.30

0.30

$-0.26$

$\begin{array}{ll}0.59 & 0.18\end{array}$

$394-0.28$

$325 \quad 0.31$

$0.31 \quad 0.21$

$-1.24$

$-1.09$

0.60

0.27
-1.39

$0.19^{1}$

0.27

$-0.66$

$-0.67$

$-0.44$

$-0.17$

440

$-0.65$

$-0.75$

$-0.65$

$-0.63$

$-0.27$

568

$\begin{array}{ll}-0.50 & -0.54\end{array}$

$\begin{array}{llll}237 & -1.00 & -0.88\end{array}$

$569-0.46$

\begin{tabular}{ll}
$77-1.47$ \\
\hline
\end{tabular}

57

$-0.34-0.58$

$-1.04$

$-0.36$

$-1.44$

$-0.26$

322

322

0.63

0.33

0.81

$-0.01$

$-0.47$

$-0.26^{1}$

$-0.08$

0.79

$\begin{array}{lllll}181 & -1.18 & -1.11 & & -1.15 \\ 181 & -0.74 & -0.90 & -1.00^{1} & -0.71\end{array}$

$\begin{array}{rrrrr}181 & -0.74 & -0.90 & -1.00^{1} & -0.71 \\ 569 & 0.00 & -0.50 & -0.13^{1} & -0.20\end{array}$

$-0.60$

$\log \mathbf{g f} \lambda$

$\log$

$\mathrm{gA} / \lambda$

4.30

5.13

5.09

5.56

6.64

$\begin{array}{rrr}-1.44 & 2.11 & 3.73 \\ 0.65 & 4.20 & 5.82\end{array}$

$\begin{array}{lll}-0.41 & 3.14 & 4.75\end{array}$

$\begin{array}{lll}-0.06 & 3.49 & 5.10\end{array}$

$\begin{array}{lll}-0.14 & 3.41 & 5.02\end{array}$

$\begin{array}{rrr}0.68 & 4.23 & 5.84 \\ -0.95 & 2.60 & 4.21\end{array}$

$\begin{array}{lll}-0.18 & 3.37 & 4.98\end{array}$

$0.15 \quad 3.70$

$0.40 \quad 3.95$

$\begin{array}{ll}0.40 & 3.95 \\ 0.40 & 3.95\end{array}$

5.31
5.56

5.56

5.56

$-0.48 \quad 3.07$
-0.71

$-0.71 \quad 2.84$

$0.60 \quad 4.15$

$-0.10 \quad 3.45$

$0.00 \quad 3.55$

$0.70 \quad 4.25$

2.95

$\begin{array}{ll}0.10 & 3.65\end{array}$

$0.81 \quad 4.36$

4.68

4.45

5.76

5.16

5.86

4.56

5.26 
TABLE 3.-Oscillator Strengths for Ultraviolet Lines of Fe 1-Continued

Wave- Low

length Energy Levels e.p. K Volts

Mult. Arc Spk CW(N) HC R(N) KOC MS Best

$\log$

No. $-\log g f-\log g f \lambda \operatorname{gA} / \lambda$

$3598.721 \quad 26225-54005 \quad 3.25$

$3598.930 \quad 26479-54258 \quad 3.28$

$3598.980 \quad 23270-51048 \quad 2.88$

$\begin{array}{lll}3599.624 & 28820-56593 & 3.57\end{array}$

$3602.080 \quad 23245-50999 \quad 2.88$

$\begin{array}{rrrrrr}674 & -0.35 & & & -0.73 & \\ 568 & & & & -1.02 & \\ 322 & -0.73 & & & -0.59 & \\ 809 & 0.31 & 0.35 & 0.34^{1} & 0.45 & \\ 322 & -0.54 & -0.66 & & -0.18 & 0.00^{1} \\ 322 & & & & 0.13 & \\ 324 & 0.26 & 0.11 & 0.20^{1} & 0.20 & \\ 370 & -1.16 & & & & \\ 295 & 0.71 & 0.73 & 0.75^{2} & 0.80 & \\ 181 & -1.53 & & & -1.24 & \\ & & & & & \\ 496 & -1.24 & & & 0.22 & \\ 323 & -0.73 & 0.00 & & & \\ & -0.46 & & & -0.30 & \\ & 0.07 & & & & \\ 294 & 1.20 & 0.94 & 0.99^{2} & 1.11 & \\ 294 & 1.24 & 1.27 & 1.05^{2} & 0.99 & \\ 325 & -0.10 & -0.20 & -0.99^{1} & 0.29 & \\ 23 & & & 0.30^{1} & 0.27 & 0.21^{3} \\ 322 & -0.77 & & & & \\ 321 & 1.10 & 0.77 & 1.13^{2} & 1.01 & 1.18^{1} \\ 323 & -0.23 & -0.39 & & -0.21 & \\ 325 & 0.26 & -0.08 & 0.26^{1} & 0.56 & \\ 46 & -1.59 & -1.66 & & -1.11 & \\ 324 & -0.56 & -0.78 & & -0.50 & \end{array}$

-0.54
-1.02
-0.66
0.36

3.02

4.62

$3602.460 \quad 23111-50862 \quad 2.86$

$\begin{array}{lll}3602.534 & 23111-50861 & 2.86\end{array}$

3602.7

$\begin{array}{ll}23111-50861 & 2.86 \\ 22838-50587 & 2.83\end{array}$

$\begin{array}{lll}3603.207 & 21716-49461 & 2.69\end{array}$

$3603.572 \quad 19621-47363 \quad 2.43$

$3603.673 \quad 20641-48383 \quad 2.56$

$3603.828 \quad 24772-52512 \quad 3.07$

$\begin{array}{lll}3604.383 & 23245-50981 & 2.88\end{array}$

$\begin{array}{lll}3604.701 & 26624-54357 & 3.30\end{array}$

$3605.206 \quad 26628-54357 \quad 3.30$

$3605.450 \quad 21999-49727 \quad 2.73$

$\begin{array}{lll}3606.682 & 21716-49434 & 2.69\end{array}$

$3608.146 \quad 22997-50704 \quad 2.85$

$\begin{array}{rrr}3608.861 & 8155-35856 & 1.01\end{array}$

$3609.486 \quad 23111-50808 \quad 2.86$

3610.159

$22650-50342 \quad 2.81$

$\begin{array}{lll}3610.703 & 23193-50880 & 2.87\end{array}$

$3612.068 \quad 22846-50523 \quad 2.83$

$\begin{array}{lll}3612.940 & 12561-40231 & 1.56\end{array}$

$3613.110 \quad 23193-50861 \quad 2.87$

$3613.459 \quad 26225-53892 \quad 3.25$

$3613.612 \quad 26624-54289 \quad 3.30$

$3614.109 \quad 26628-54289 \quad 3.30$

$3614.711 \quad 26225-53882 \quad 3.25$

$3615.190 \quad 26479-54132 \quad 3.28$

3615.665

3615.959

3616.162

3616.326

3617.090

$11976-39626 \quad 1.48$

$26624-54271 \quad 3.30$

$25900-53546 \quad 3.21$

$\begin{array}{ll}19552-47197 & 2.42\end{array}$

3617.788

$24575-52213 \quad 3.05$

672

$-0.12$

$-0.62-0.59$

$-0.29-0.51$

3618.285

$24336-51969 \quad 3.02$

3618.392

$22846-50475 \quad 2.83$

$21999-49628 \quad 2.73$

3618.769

$\begin{array}{rr}7986-35612 & 0.99\end{array}$

3619.772

$19390-47008 \quad 2.40$

3620.228

$22997-50611 \quad 2.85$

$3620.880 \quad 23270-50880 \quad 2.88$

$\begin{array}{lll}3621.464 & 21999-49604 & 2.73\end{array}$

$3621.718 \quad 28820-56423 \quad 3.57$

3622.001

$22249-49851 \quad 2.76$

3623.188

$19390-46982 \quad 2.40$

$-0.14$

$569-1.29$

$-0.83$

$\begin{array}{llll}46 & -1.79 & -1.88 & -1.43\end{array}$

$-0.64$

$\begin{array}{lll}569 & -0.48 & -0.60\end{array}$

$\begin{array}{llll}132 & -1.06 & -0.93 & -0.93 \\ 535 & & & -0.77\end{array}$

$.00^{1}$

2.54

4.14

4.50

0.13

3.92

5.52

3623.440

3623.772

3624.056

3624.310

$20641-48231 \quad 2.56$

$23111-50699 \quad 2.86$

$26340-53925 \quad 3.26$

3625.140

$19552-47136 \quad 2.42$

496

$0.87^{2}$

0.97

324
295

$-0.76$

0.89

0.28

$0.27^{1}$

0.67

$-1.06$

$-0.93$

$0.26^{3}$

$-0.72$

$324-0.85$

$\begin{array}{rr}323 & -0.96 \\ 294 & 0.94\end{array}$

0.94
-0.09

0.95

1.03

$\begin{array}{ll}0.82 & 0.81\end{array}$

$0.97^{2}$

0.48

$\begin{array}{ll}0.80^{2} & 0.87\end{array}$

0.19

3.69

5.28

$-1.16$

3.75

5.34

$\begin{array}{rrr}0.75 & 4.31 & 5.90 \\ -1.38 & 2.18 & 3.77\end{array}$

4.31

3.99

$\begin{array}{lll}-1.24 & 2.32 & 3.91\end{array}$

0.02

2.32
3.58

$-0.74$

2.82

5.17

$-0.38$

3.18

4.77

4.77

$\begin{array}{lll}1.05 & 4.61 & 6.20\end{array}$

$\begin{array}{lll}1.14 & 4.70 \quad 6.29\end{array}$

$.21^{3}$

$\begin{array}{rrr}0.25 & 3.81 & 5.40 \\ -0.77 & 2.79 & 4.38\end{array}$

$\begin{array}{lll}1.05 & 4.61 & 6.20\end{array}$

$\begin{array}{rr}-0.28 & 3.28-4.87\end{array}$

$\begin{array}{lll}0.15 & 3.71 & 5.30\end{array}$

$\begin{array}{lll}-1.62 & 1.94 & 3.53\end{array}$

$\begin{array}{lll}-0.61 & 2.95 & 4.54\end{array}$

$\begin{array}{lll}-0.12 & 3.44 & 5.03\end{array}$

$\begin{array}{lll}-0.60 & 2.96 & 4.55\end{array}$

$\begin{array}{lll}-0.40 & 3.16 & 4.75\end{array}$

$\begin{array}{lll}-0.14 & 3.42 & 5.01\end{array}$

$28820-56383 \quad 3.57$

$\begin{array}{lll}3628.806 & 24119-51668 & 2.99\end{array}$

$3630.353 \quad 22997-50534 \quad 2.85$

$180 \quad 0.27$

$\begin{array}{lll}0.17 & 0.25^{2} & 0.26\end{array}$

0.26
-0.23
-

$-0.55 \quad-0.55$

$-0.01$

$570-0.63$

$-0.59$

$-0.83-0.88$

$-0.54$

$-1.84$

1.72

3.31

$-1.12$

2.44

4.03

$-0.57$

$-0.97$

(.9.

$-0.77$

2.79

4.18

$\begin{array}{lll}0.89 & 4.45 & 6.04\end{array}$

$-0.76$

4.45

4.39

$\begin{array}{lll}0.18 & 3.74 & 5.33\end{array}$

$\begin{array}{lll}0.28 & 3.84 & 5.43\end{array}$

$\begin{array}{lll}-1.00 & 2.56 & 4.15\end{array}$

$\begin{array}{lll}-0.78 & 2.78 \quad 4.37\end{array}$

$\begin{array}{lll}0.97 & 4.53 \quad 6.12\end{array}$

$\begin{array}{lll}0.04 & 3.60 & 5.19\end{array}$

$\begin{array}{lll}0.82 & 4.38 & 5.97\end{array}$

$\begin{array}{lll}0.24 & 3.80 & 5.39\end{array}$

$\begin{array}{lll}-0.44 & 3.12 & 4.71\end{array}$

$\begin{array}{lll}-0.63 & 2.93 \quad 4.52\end{array}$

$\begin{array}{lll}-0.75 & 2.81 \quad 4.40\end{array}$

$\begin{array}{rrrrr}323 & 0.17 & -0.07 & 0.29^{2} & 0.43 \\ 808 & -0.53 & -0.41 & & -0.03\end{array}$

$\begin{array}{lll}0.17 & 3.73 & 5.32\end{array}$

$\begin{array}{lll}-0.47 & 3.09 & 4.68\end{array}$

$\begin{array}{lll}-1.11 & 2.45 & 4.03\end{array}$

$\begin{array}{lll}-1.05 & 2.51 & 4.09\end{array}$

$3631.103 \quad 22846-50378 \quad 2.83$

$3631.465 \quad 7728-35257 \quad 0.96$

$3632.042 \quad 24772-52297 \quad 3.07$

$\begin{array}{lll}3632.558 & 23784-51305 & 2.95\end{array}$

3632.980

$20038-47556 \quad 2.48$

$\begin{array}{lll}738 & -1.04 & -1.18 \\ 323 & -0.02 & -0.95\end{array}$

$\begin{array}{lllll}323 & 0.02 & -0.20 & 0.04^{1} & 0.26\end{array}$

$-0.05$

3.51

5.09

$\begin{array}{rrrrr}322 & 0.61 & & & 0.83 \\ 23 & & & 0.23^{1} & 0.53 \\ 496 & 0.79 & 0.75 & 0.71^{1} & 0.78 \\ 437 & -0.02 & -0.01 & & -0.28 \\ 135 & -0.40 & -0.41 & -0.19^{1} & -0.25\end{array}$

$0.10^{1}$

$\begin{array}{lll}0.22 & 3.78 & 5.36\end{array}$

$\begin{array}{lll}0.76 & 4.32 & 5.90\end{array}$

$\begin{array}{lll}-0.08 & 3.48 & 5.06\end{array}$

$\begin{array}{lll}-0.31 & 3.25 & 4.83\end{array}$ 
TABLE 3.-Oscillator Strengths for Ultraviolet Lines of Fe 1 -Continued

Wave-

Wave- Low

A $\quad K \quad$ Volts

$\begin{array}{lll}3633.087 & 23711-51229 & 2.94 \\ 3633.833 & 24119-51630 & 2.99 \\ 3634.326 & 23711-51219 & 2.94 \\ 3635.190 & 24336-51837 & 3.02 \\ 3636.186 & 17727-45221 & 2.20\end{array}$

$3636.496 \quad 26340-53831 \quad 3.26$

$3636.650 \quad 24336-51826 \quad 3.02$

$3636.995 \quad 20875-48362 \quad 2.59$

$3637.251 \quad 19621-47107 \quad 2.43$

3637.730

3637.862

3638.300

3640.392

3641.454

3643.627

3643.716

3643.812

3644.798

3645.090

3645.494

3645.822

3647.427

3647.844

3649.304

3649.509

3649.699

3650.031

3650.281

3650.554

3651.100

3651.470

3652.256

3653.352

3653.763

3654.660

3655.467

3656.227

3656.358

3657.139

3657.890

3658.025

3658.550

3659.519

3660.330

3661.360

3663.250

3663.458

3663.950

3664.537

3664.694

3666.240

3666.944

3667.252

3667.999

3668.214

$\begin{array}{lll}3668.893 & 20875-48123 & 2.59\end{array}$

$\begin{array}{lll}3669.151 & 24119-51365 & 2.99\end{array}$

$\begin{array}{lll}3669.523 & 21999-49243 & 2.73\end{array}$

$\begin{array}{lll}3670.071 & 23784-51023 & 2.95\end{array}$

3670.810
$23711-51192 \quad 2.94$ $2249-49727 \quad 2.76$ $21999-49461 \quad 2.73$

$23245-50699 \quad 2.88$

$21039-48476 \quad 2.61$

$12969-40405 \quad 1.61$

$26140-53569 \quad 3.24$

$22997-50423 \quad 2.85$

$25092-52512 \quad 3.11$

$12561-39970 \quad 1.56$

$\begin{array}{ll}0-34782 & 0.91\end{array}$

$\begin{array}{rr}21716-49109 & 2.69\end{array}$

$23711-51103 \quad 2.94$

$24181-51570 \quad 3.00$

$\begin{array}{ll}19621-47008 & 2.43 \\ 26225-53610 & 3.25\end{array}$

$22997-50378 \quad 2.85$

$22249-49628 \quad 2.76$

3.02

$19621-46982 \quad 2.43$

$17927-45282 \quad 2.22$

$22838-50187 \quad 2.83$

3.27

$\begin{array}{ll}23193-50534 & 2.87 \\ 19552-46889 & 2.42\end{array}$

$24507-51837 \quad 3.04$

$24339-51668 \quad 3.02$

$19788-47107 \quad 2.45$

$23111-50423 \quad 2.86$

$19788-47093 \quad 2.45$

$24119-51409 \quad 2.99$

$20875-48163 \quad 2.59$

$23784-51069 \quad 2.95$

$24181-51461 \quad 3.00$

$12969-40231 \quad 1.61$

$25900-53161 \quad 3.21$

$25900-53155 \quad 3.21$

$26140-53394 \quad 3.24$

$20038-47272$

2.48
$19621-46889 \quad 2.43$
Mult.
No.

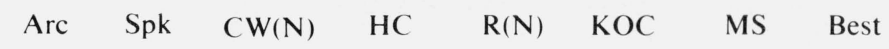

$\begin{array}{rrrrrr}390 & -0.04 & & & -0.18 & \\ 440 & -0.36 & -0.49 & & -0.14 & \\ 389 & 0.12 & -0.02 & 0.35^{1} & 0.38 & \\ 490 & -0.17 & -0.45 & & 0.09 & \\ 77 & & -0.46 & -0.40^{1} & -0.55 & \\ & & & & & \\ 568 & -0.49 & & & & \\ 493 & & -0.32 & & -0.30 & \\ 233 & -0.39 & -0.49 & & -0.08 & \\ 180 & -0.66 & -0.90 & & -0.65 & \\ 229 & -1.29 & -0.75 & & -0.72 & \\ & & & & 0.13 & 0.09^{1} \\ 385 & -0.10 & -0.38 & & 0.69 & \\ 294 & 0.66 & 0.58 & 0.64^{2} & 0.95 & \\ 295 & 0.86 & 0.86 & 0.89^{2} & 0.33^{1} \\ 323 & -1.25 & -0.83 & 0.24^{1} & 0.28 & 0.28 \\ 385 & 0.08 & 0.00 & 0.25 & \\ 233 & -0.21 & & & -0.22 & \\ 46 & -1.46 & & & 0.17 & \\ 570 & -0.22 & -0.59 & & & \\ 323 & -0.26 & & -0.15^{1} & -0.05 & \\ 323 & -0.42 & -0.40 & & 0.04 & \\ & & & & & \\ 496 & 0.50 & 0.48 & 0.59^{2} & 0.67 & \\ 46 & -1.17 & -0.87 & & & \\ 23 & & & 0.09^{2} & 0.38 & 0.08^{3} \\ 5 & -2.34 & -2.45 & & -2.59 & -2.75^{1} \\ 291 & 0.88 & 0.87 & 0.78^{2} & 1.04 & \end{array}$

$391-0.60$

394

180

322

0.32

0.21

$-0.42$

0.58

0.08

0.61

0.39

295

$-0.76$

0.52

$-0.44^{1}$

$\begin{array}{rr}1.00 & 0.98 \\ & -0.85\end{array}$

$229-1.48$

$180-0.72$

$-0.83$

$-1.61-1.53$

1.06

$-0.77$

$-1.29$

$\begin{array}{lllll}369 & 0.10 & 0.12 & 0.21^{1} & 0.18\end{array}$

$0.01-0.28$
-1.05

323

130

395

$-1.05$

$-0.71$

$\begin{array}{lll}-0.81 & -0.46^{1} & -0.57\end{array}$

$-0.18$

$-0.58$

$-0.1$

0.55

231

180

323

$-1.27$

$-1.21$

$-1.03$

$-1.23$

$-1.06$

0.2

$\begin{array}{rr} & -0.86 \\ .20^{2} & 0.19\end{array}$

$-0.92$

$439-0.51 \quad-0.63$

$-0.34$

$\begin{array}{lll}231 & -0.60 & -0.83\end{array}$

$\begin{array}{lll}435 & -0.90 & -0.83\end{array}$

$391-0.08$

390

-0.08
-0.45

$-0.54$

$179-0.76$

46

570

569

568

$-2.25$

$\begin{array}{ll}0.21 & -0.29\end{array}$

$\begin{array}{ll}-0.03 & -0.49\end{array}$

$\begin{array}{ll}-0.29 & -0.57\end{array}$

229

$-1.30$

437
291

291
435

0.09

$-0.02$

$\begin{array}{ll}0.65 & 0.57\end{array}$

0.26

$-0.73 \quad-0.83$

133

$-0.28$

$-0.19^{1} \quad 0.00$

$-0.26$

$-0.84^{1}-0.56$

$-1.01$

$\begin{array}{ll}0.17^{1} & 0.00\end{array}$

$-0.09$

$-0.33$

$-0.11$

-0.33
0.21

-0.31
-0.47

$-0.49$

$-0.31$

$-0.32$

$-0.74$

0.04

0.64

0.89

$-1.25$

0.19

$-0.22$

$-0.40$

$-0.15$

$-0.41$

0.57

$-1.02$

$-2.53$

0.75

0.85

$-0.60$

0.23

$-0.42$

$-0.60$

1.00
-0.85

$-1.48$

$-0.77$

0.15

$-0.14$

$-1.05$

$-0.64$

$-0.14$

0.55

$-1.1$

$-1.03$

$-1.07$

$-0.49$

$-0.72$

$-0.86$

$-0.09$

$-0.42$

$-0.72$

$-2.25$

$-0.40$

$-1.10$

$\begin{array}{ll}0.69^{2} & 0.10 \\ & \end{array}$

$-0.69$

0.26

3.25

3.24
2.82

3.60

4.20

4.45

2.31

3.34

3.16

3.41
3.15

4.13

4.13
2.54

2.54
3.70

1.03

4.41

2.96

4.06

3.79

3.14
2.96

4.56

4.56
2.71

2.08

2.79

$-1.48 \quad 2.08$

3.71

3.42

2.51

2.92
3.42

4.11

2.45

3.70

2.53
2.49

3.07

2.84

2.70

\begin{tabular}{l}
3.14 \\
\hline
\end{tabular}

2.84

1.31

4.41

3.16

4.73

$-1.20 \quad 2.36$

$0.06 \quad 3.62$

$0.65 \quad 4.21$

3.82

3.93

5.19

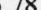

5.39
4.38 
TABLE 3.-Oscillator Strengths for Ultraviolet Lines of Fe I-Continued

Wave- $\quad$ Low length

A

Energy Levels e.p. K Volts

$3671.510 \quad 26340-53569 \quad 3.26$

$3671.689 \quad 22249-49477 \quad 2.76$

$19788-47008 \quad 2.45$

$3674.766 \quad 22838-50043 \quad 2.83$

$3675.434 \quad 21039-48239 \quad 2.61$

$3675.694 \quad 24507-51705 \quad 3.04$

$3676.314 \quad 20641-47835 \quad 2.56$

$3676.879 \quad 24181-51370 \quad 3.00$

$\begin{array}{lll}3677.309 & 28605-55791 & 3.55\end{array}$

3677.477

3677.631

3678.862

3678.980

3679.330

3679.530

3679.915

3680.675

3681.227

3681.651

3681.880

3682.226

3683.056

3683.616

3684.110

3685.998

3686.260

3687.098

3687.459

3687.656

3688.198

3688.476

3689.010

3689.457

3689.897

3690.450

3690.730

3693.008

3694.005

3695.054

3695.507

3696.030

3697.426

3697.536

3698.611

3699.147

3701.086

3702.033

3702.500

3703.556

3703.697

3703.824

3704.021

3704.464

3705.567

3707.048

3707.458

3707.578

3707.823

3707.922

3708.602
$18378-45563 \quad 2.28$

$22249-49433 \quad 2.76$

$19552-46727 \quad 2.42$

$18378-45552 \quad 2.28$

$20641-47812 \quad 2.56$

$24181-51351 \quad 3.00$

$25900-53061 \quad 3.21$

$26628-53785 \quad 3.30$

$24181-51335 \quad 3.00$

$29799-56951 \quad 3.69$

$28605-55754 \quad 3.55$

$416-27560 \quad 0.05$

$20038-47177 \quad 2.48$

$21999-49135 \quad 2.73$

$23711-50833 \quad 2.94$

$19552-46673 \quad 2.42$

$17550-44664 \quad 2.18$

6928-34040 0.86

$21999-49109 \quad 2.73$

$26628-53734 \quad 3.30$

$26225-53329 \quad 3.25$

$19621-46721 \quad 2.43$

$23711-50808 \quad 2.94$

$24575-51668 \quad 3.05$

$25092-52181 \quad 3.11$

$28820-55907 \quad 3.57$

$24339-51409 \quad 3.02$

$24507-51570 \quad 3.04$

$20875-47930 \quad 2.59$

$20641-47693 \quad 2.56$

$19552-46601 \quad 2.42$

$24181-51219 \quad 3.00$

$26624-53661 \quad 3.30$

$24336-51365 \quad 3.02$

$24336-51361 \quad 3.02$

$24181-51192 \quad 3.00$

$22947-49951 \quad 2.84$

$12969-39970 \quad 1.61$

$22249-49243 \quad 2.76$

$23711-50704 \quad 2.94$

23052-50043 2.86

$24772-51762 \quad 3.07$

$21716-48703 \quad 2.69$

$416-27395 \quad 0.05$

$24181-51149 \quad 3.00$

$20641-47606 \quad 2.56$

$31307-58272 \quad 3.88$

$19788-46745 \quad 2.45$
$0-27167 \quad 0.00$

$\begin{array}{ll}704-27666 & 0.09\end{array}$

$17550-44512 \quad 2.18$

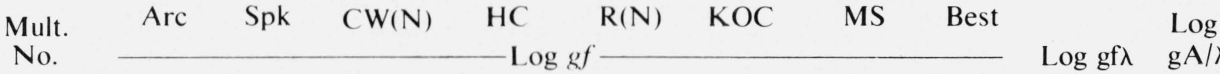

$\begin{array}{cccccccccc}\begin{array}{c}\text { Mult. } \\ \text { No. }\end{array} & \text { Arc } & \text { Spk } & \mathrm{CW}(\mathrm{N}) & \mathrm{HC} & \mathrm{R}(\mathrm{N}) & \mathrm{KOC} & \mathrm{MS} & \text { Best } & \log \\ & & & & & & & \end{array}$

$\begin{array}{cccccccccc}\begin{array}{c}\text { Mult. } \\ \text { No. }\end{array} & \text { Arc } & \text { Spk } & \mathrm{CW}(\mathrm{N}) & \mathrm{HC} & \mathrm{R}(\mathrm{N}) & \mathrm{KOC} & \mathrm{MS} & \text { Best } & \log \\ & & & & & & & \end{array}$

\begin{tabular}{|c|c|c|c|c|c|c|c|c|}
\hline 570 & $\begin{array}{l}-0.97 \\
-1.18\end{array}$ & -102 & & & & & $\begin{array}{l}-0.97 \\
-1.10\end{array}$ & $\begin{array}{l}2.59 \\
2.46\end{array}$ \\
\hline 180 & -0.88 & -1.03 & & -0.89 & & & -0.93 & $\begin{array}{l}2.40 \\
2.63\end{array}$ \\
\hline 369 & -0.17 & -0.33 & $-0.03^{1}$ & -0.20 & & & -0.18 & 3.39 \\
\hline 229 & -1.89 & & & & & & -1.89 & 1.68 \\
\hline 391 & -1.29 & -1.14 & & & & & -1.22 & 2.35 \\
\hline 228 & 0.13 & 0.04 & $0.15^{1}$ & 0.14 & & & 0.12 & 3.69 \\
\hline 389 & -0.75 & -0.74 & & -0.42 & & & -0.64 & 2.93 \\
\hline 773 & 0.35 & 0.45 & & 0.97 & & & 0.40 & 3.97 \\
\hline 125 & -0.80 & -0.84 & & -0.85 & & & -0.83 & 2.74 \\
\hline 291 & 0.89 & 0.78 & $0.85^{2}$ & 0.65 & & & 0.80 & 4.37 \\
\hline 131 & -0.30 & -0.41 & $-0.28^{1}$ & -0.15 & & & -0.28 & 3.29 \\
\hline 124 & -1.21 & & & -0.95 & & & -1.08 & 2.49 \\
\hline 228 & & & & -1.13 & & & -1.13 & 2.44 \\
\hline 393 & & & & -0.45 & & & -0.45 & 3.12 \\
\hline 5 & & -1.22 & $-1.27^{2}$ & -1.05 & $-1.37^{4}$ & -1.33 & -1.30 & 2.27 \\
\hline 568 & 0.14 & & $0.35^{1}$ & 0.14 & & & 0.21 & 3.78 \\
\hline & -0.36 & $-0.4 /$ & & & & & -0.42 & 3.15 \\
\hline 51 & -0.58 & & & $\begin{array}{l}-0.4 / \\
-0.26\end{array}$ & & & $\begin{array}{l}-0.32 \\
-0.26\end{array}$ & $\begin{array}{l}3.05 \\
3.31\end{array}$ \\
\hline
\end{tabular}

$\begin{array}{lllll}772 & 1.16 & 0.97 & 1.24^{2} & 1.29\end{array}$

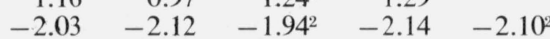

1.18

$-2.05$

$\begin{array}{rrr}5 & -2.03 & -2.12 \\ 130 & -1.32 & -1.19\end{array}$

$0.63^{2} \quad 0.72$

$\begin{array}{lll}0.78^{2} & 0.81 & 0.76^{1}\end{array}$

0.65

0.74

$131-0.53 \quad-0.48$

$\begin{array}{lll}75 & -0.70 & -0.80\end{array}$

$\begin{array}{rrr}21 & -0.43 & -0.31 \\ 291 & 0.58 & 0.31\end{array}$

$\begin{array}{rrr} & -0.49 & \\ -0.42^{2} & -0.11 & -0.61^{2} \\ & 0.35 & \end{array}$

$-0.50$

$-0.75$

$-0.47$

$-1.04$

$-1.04$

$-0.30$

$-1.72$

0.72

$0.81^{2}-0.81$
-0.70

$0.72^{1}$

$386 \quad 0.75$

0.42

-0.70
-0.30

$-0.30$

0.54

$-0.24$

1.10

0.36

$-1.20$

$-2.05$

0.28

$-0.04$

$-0.17$

$-0.62$

1.01

$-0.10$

$-1.63$

0.07

0.33

$-0.06$

$-0.76$

0.41

$\begin{array}{rrrrr}495 & -0.98 & -0.69 & & -0.62 \\ 290 & 0.42 & 0.32 & 0.42^{2} & 0.49\end{array}$

$-1.04$

$\begin{array}{lll}-1.12^{3} & -0.90 & -1.15^{4}\end{array}$

0.53

$\begin{array}{rr}-0.90 & -1.15^{4} \\ 0.76 & 0.53^{1}\end{array}$

$-1.09$

$-1.02$

$-1.71$

0.64

$-1.53^{1}$

-1.75
0.79

$-1.95^{2}$

$-0.70$
4.75

0.77

1.52

2.31

4.22
4.34

\subsection{7}

2.82
3.10

0.4

3.98

2.53

3.27

1.85

4.29

2.74
3.27

4.11

3.33

4.67

3.93

2.37

1.52
3.85

3.85

3.40

0.18

$-1.10$

\subsection{8}

3.47

1.94

3.64

3.75

3.51

2.81

3.98

2.47

4.18

0.14

2.55

3.71

4.16

4.95

3.91

5.25
4.49

5.53

4.30

5.93

4.85

4.00

4.68

3.83

4.61

6.31

3.08

3.87

5.77
5.89

4.62

4.37

4.65

5.53

4.08

4.82

3.40

5.84

4.82

5.66

4.88

6.22

5.48

3.92

3.07

5.40

5.08

4.95

6.13

5.02

3.49

5.19
5.30

5.06

4.36

5.53

4.02
5.73

4.10

5.26

$\begin{array}{lll}0.72 & 4.29 & 5.84\end{array}$ 
TABIE 3.-Oscillator Strengths for Ultraviolet Lines of Fe I-Continued

Wave-

length Energy Levels Low.

A

3709.248

$3709.535-24119-51069-2.99$

$3709.665 \quad 20641-47590 \quad 2.56$

$\begin{array}{lll}3711.224 & 20875-47812 \quad 2.59\end{array}$

$3711.411 \quad 24772-51708 \quad 3.07$

$\begin{array}{lll}3715.914 & 18378-45282 & 2.28\end{array}$

$3716.442 \quad 23711-50611 \quad 2.94$

$\begin{array}{lll}3717.837 & 17550-54449 & 2.18\end{array}$

$\begin{array}{lll}3718.409 & 22249-49135 & 2.76\end{array}$

$\begin{array}{lll}3719.937 & 0-26875 & 0.00\end{array}$

$3721.189 \quad 24336-51201 \quad 3.02$

3721.278

3721.396

3721.510

3721.606

3722.026

3722.564

3724.380

3725.498

3726.927

3727.096

3727.621

3727.809

3728.670

3730.388

3730.945

3731.376

3732.399

3733.319

3734.866

3735.325

3737.133

3738.308

3739.120

3739.317

3740.061

3740.247

3741.486

3742.151

3742.621

3742.937

3743.364

3743.781

3744.105

3745.562

3745.901

3746.486

3746.931

3748.264

3748.492

3748.969

3749.488

3750.677

3751.059

3751.820

3752.420

3753.154

3753.613

3754.506

3756.069
$20038-46902 \quad 2.48$

$24507-51370 \quad 3.04$

$24339-51201 \quad 3.02$

$22249-49109 \quad 2.76$

$704-27560 \quad 0.09$

$\begin{array}{ll}18378-45221 & 2.28\end{array}$

$24575-51409 \quad 3.05$

$24507-51331 \quad 3.04$

$23711-50534 \quad 2.94$

$7728-34547 \quad 0.96$

$24181-50999 \quad 3.00$

$24575-51374 \quad 3.05$

$21039-47834 \quad 2.61$

$21039-47831 \quad 2.61$

$17727-44512 \quad 2.20$

$888-27666 \quad 0.11$

$6928-33695 \quad 0.86$

$23711-50475 \quad 2.94$

$416-27167 \quad 0.05$

$26351-53094 \quad 3.27$

$17927-44664 \quad 2.22$

$17550-44285 \quad 2.18$

$27395-54125 \quad 3.40$

$26225-52954 \quad 3.25$

$27666-54386 \quad 3.43$

$31805-58520 \quad 3.94$

$23711-50423 \quad 2.94$

$27666-54376$

$7986-34692$

$21999-48703$

$24507-51208$

$704-27395 \quad 0.09$

$978-27666 \quad 0.12$

$17727-44411 \quad 2.20$

$24181-50862 \quad 3.00$

$888-27560 \quad 0.11$

$28820-55490 \quad 3.57$

$23711-50378 \quad 2.94$

$7377-34040 \quad 0.91$

$21039-47693 \quad 2.61$

$26624-53275 \quad 3.30$

$21716-48362 \quad 2.69$

$24507-51149 \quad 3.04$

$19390-46027 \quad 2.40$

$17550-44184 \quad 2.18$

$24181-50808 \quad 3.00$

$\begin{array}{ll}17550-44166 & 2.18\end{array}$
$26875-53739 \quad 3.33$

$20641-47453 \quad 2.56$
Arc Spk CW(N) HC R(N) KOC MS Best

Mult. No.

$\begin{array}{rrrrr} & & & & \\ & & & & \\ & -0.20 & -0.26^{3} & 0.05 & -0.28^{1} \\ -0.17 & 0.02 & & & \\ -0.63 & & & & \\ -0.19 & -0.22 & 0.03^{1} & 0.19 & \\ -0.07 & -0.18 & & 0.03 & \\ -0.49 & -0.59 & -0.39^{1} & -0.38 & \end{array}$

$$
21
$$

435

225

228

494

124

388

706
292

0.58

292
5

491

705
131

389
437

291

124

53

385

387

21
386

386
227

533

228

22

76

21

388

$-0.13$

$-0.14$

$\begin{array}{rrr}0.02^{1} & 0.20 & \\ -0.36^{3} & -0.21 & -0.34^{2}\end{array}$

$\begin{array}{rrr}0.02^{1} & 0.20 & \\ -0.36^{3} & -0.21 & -0.34^{2}\end{array}$

$-0.48$

0.03

$-0.72$

$-0.03$

$-0.68$

0.13
-0.40

0.40

$-0.04$

$-0.34^{2}$

$-0.06$

$-0.46$

0.27

$-0.96$

$-1.083$

0.00

$\begin{array}{rrrr}0.27 & 0.05 & 0.21^{2} & 0.35 \\ -0.56 & -0.56 & -0.55^{1} & 0.56\end{array}$

0.53

0.29

$0.26^{1}$

0.06

0.36

0.14

$-0.68$

0.31

$-0.73$

$-0.25^{3}$

0.59

$0.16-0.38^{2}$

$\begin{array}{ll}0.41 & 0.24^{1}\end{array}$

0.29

$-0.60^{1}$

$-0.44$

$\begin{array}{ll}0.43^{1} & 0.34\end{array}$

-0.24
0.20

$-0.47-0.34$

$0.31-0.38$

$-0.33^{1}$

$0.30^{2}$

0.42

$-1.08$

$-1.22^{3}$
$0.55^{3}$

$-1.18$

$-1.16^{2}$

$\begin{array}{rrr} & 0.43 & \\ -0.49^{3} & -0.67 & -0.46^{2}\end{array}$

0.79

$\begin{array}{rr}0.79 & 0.83 \\ -1.36 & -1.41\end{array}$

$0.82^{2}$

0.83

$-1.36$

$\begin{array}{ll}75 & -1.36 \\ 74 & -1.48\end{array}$

.

707

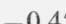

$-0.50$

0.2

$-0.70$

667

701

0.08

$-0.97$

-0.53
0.37

0.27

$0.27^{1}$

0.07

$-1.41$

70

21
290

385

$-0.76$

$-0.35$

$-0.27$

(20.

0.19

$-0.24$

$0.31^{1}$

$-0.72$

0.12

$-0.62^{2}$

1.17

$-0.61^{2}$

$-1.09$

$-1.15^{2}$

$-1.17$

$-1.10^{2}$

$-1.28-1.15$

$0.41^{1}$

$-0.87^{3}$

$-0.38$

$-0.93$

$0.35^{1}$
$-0.86^{2}$

805

386

21

225

667

287

385

177

73

386

$0.47 \quad 0.02$

$-1.26-0.82$

$0.43^{3}$

0.45

$\begin{array}{ll}-1.03 & -0.98\end{array}$

-0.62
-0.30

$0.37^{1}$

$\begin{array}{ll}-0.62 & -0.70\end{array}$

$\begin{array}{ll}-1.45 & -1.39\end{array}$

$\begin{array}{ll}-0.09 & -0.20\end{array}$

$0.02^{2}$

$-0.30^{1}$

$-1.17$

$-1.28 \quad-1.03^{1}$

$-0$.

$-1.60$

$-0.11$

$-0.49 \quad-0.49$

$-0.07$

$-0.46$

0.54

$-1.64$

$-0.01$

$\begin{array}{ll}-0.31 & -0.32\end{array}$

$-0.48$

$\begin{array}{llll}-0.29 & -0.30 & 3.27 & 4.82\end{array}$

$\begin{array}{ll}-0.30 & 3.27 \\ -0.08 & 3.49\end{array}$

$\begin{array}{ll}-0.63 & 2.94\end{array}$

$-0.13 \quad 3.44$

3.50 
TABLE 3.-Oscillator Strengths for Ultraviolet Lines of Fe I-Continued

Wave-

K Volts

$\begin{array}{rrr}3756.939 & 28820-55430 & 3.5 \\ 3757.459 & 26624-53230 & 3.30 \\ 3758.235 & 7728-34329 & 0.9 \\ 3759.155 & 29313-55907 & 3.6 \\ 3759.597 & 27666-54258 & 3.4\end{array}$

$3760.052 \quad 19390-45978 \quad 2.40$

3760.533

3761.069

3761.410

3762.205

$17927-44512$

$27167-53748$

$20875-47453$

$27167-53739 \quad 3.37$

3763.791

$7986-34547 \quad 0.99$

3765.541

3765.700

3766.092

3766.665

3767.194

3768.030

3768.230

3769.995

3770.305

3770.405

3771.473

3773.364

3773.699

3774.827

3775.860

3776.455

3777.061

3777.452

3778.320

3778.509

3778.699

3779.213

3779.444

3781.188

3781.938

3782.450

3782.608

3785.706

3785.950

3786.176

3786.678

3787.164

3787.883

3789.178

3789.570

3789.808

3790.094

3790.656

3790.756

3791.504

3791.730

3792.156

3792.833

3793.360

3793.478

3793.872

3794.340

3795.004

3796.000
$26106-52655 \quad 3.24$

$26351-52899 \quad 3.27$

$20875-47420 \quad 2.59$

$24507-51048 \quad 3.04$

$8155-34692 \quad 1.01$

$17927-44459 \quad 2.22$

$22947-49477 \quad 2.84$

$24181-50699 \quad 3.00$

$21716-48231 \quad 2.69$

$19621-46136 \quad 2.43$

$26106-52613 \quad 3.24$

$24575-51069 \quad 3.05$

$24507-50999 \quad 3.04$

$17927-44411 \quad 2.22$

$21999-48476 \quad 2.73$

$17550-44023 \quad 2.18$

$24119-50587 \quad 2.99$

$20641-47107 \quad 2.56$

$22838-49298 \quad 2.83$

$26225-52683 \quad 3.25$

$17727-44184 \quad 2.20$

$22249-48703 \quad 2.76$

$26406-52858 \quad 3.27$

$17727-44166 \quad 2.20$

$29357-55791 \quad 3.64$

$24181-50611 \quad 3.00$

$24772-51201 \quad 3.07$

$26106-52514 \quad 3.24$

$19621-46027 \quad 2.43$

$22838-49243 \quad 2.83$

$8155-34556 \quad 1.01$

$29357-55754 \quad 3.64$

$8155-34547 \quad 1.01$

$21999-48383 \quad 2.73$

$21039-47420 \quad 2.61$

$7986-34363 \quad 0.99$

$24507-50880 \quad 3.04$

$17550-43923 \quad 2.18$

$20641-47008 \quad 2.56$

$27560-53925 \quad 3.42$

$21999-48362 \quad 2.73$

$17927-44285 \quad 2.22$

$24507-50861 \quad 3.04$

$24181-50534 \quad 3.00$

$22947-49298 \quad 2.84$

$19788-46136 \quad 2.45$

$7986-34329 \quad 0.99$

$19390-45726$

2.40
$27167-53546 \quad 3.37$
Mult.

Arc Spk $\quad$ WW(N)

HC R(N) KOC MS Best

$\longrightarrow \log g \mathrm{gA} \lambda / \lambda$

$\log$

No. $\quad$ Log $g$

$\begin{array}{rrrrrrrrrr}805 & 0.37 & 0.56 & 0.63^{1} & 0.42 & & & 0.50 & 4.07 & 5.6 \\ 668 & -0.18 & -0.20 & & -0.36 & & & -0.25 & 3.32 & 4.8 \\ 21 & & & 0.36^{3} & 0.27 & 0.28^{2} & 0.26 & 0.31 & 3.88 & 5.4 \\ 855 & -0.43 & -0.31 & & -0.82 & & & -0.37 & 3.21 & 4.7 \\ 701 & -2.22 & & & & & -2.22 & 1.36 & 2.8 \\ & & & & & & 0.15 & 3.73 & 5.25 \\ 177 & 0.14 & 0.08 & 0.18^{2} & 0.19 & & -0.47 & 3.11 & 4.6 \\ 76 & -0.36 & -0.42 & -0.61^{2} & -0.34 & & -1.40 & 2.18 & 3.7 \\ 706 & -1.40 & & & & & -0.95 & 2.63 & 4.1 \\ 227 & -0.90 & -0.95 & -1.06^{1} & -0.88 & & -0.69 & 2.89 & 4.4 \\ 705 & -0.54 & & & -0.84 & & & \end{array}$

$\begin{array}{llllllll}21 & 0.12^{3} & 0.17 & 0.10^{2} & 0.07 & 0.11 & 3.69 & 5.21\end{array}$

$\begin{array}{llllllll}608 & 1.19 & 1.28 & 0.98^{2} & 0.99 & 1.08 & 4.66 & 6.18\end{array}$

$\begin{array}{llllll}608 & & -0.52 & -0.52 & 3.06 & 4.58\end{array}$

$\begin{array}{lll}-1.20 & 2.38 & -3.90\end{array}$

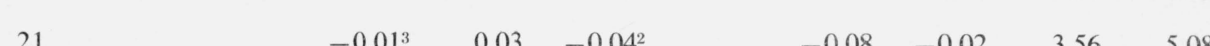

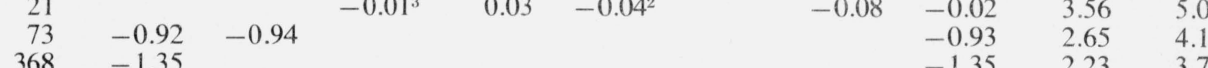

$\begin{array}{lllllll}387 & -0.41 & -0.46^{1} & -0.34 & -1.35 & 2.23 & 3.75 \\ 287 & -0.51 & & -0.53 & -0.40 & 3.18 & 4.69\end{array}$

$\begin{array}{llllll}287 & -0.51 & -0.53 & -0.52 & 3.06 & 4.57\end{array}$

$177-1.40$

$\begin{array}{lll}-1.40 & 2.18 & 3.69\end{array}$

$\begin{array}{lll}-0.99 & 2.59 & 4.10\end{array}$

$\begin{array}{lll}-1.14 & 2.44 & 3.95\end{array}$

$\begin{array}{lll}-0.62 & 2.96 & 4.47\end{array}$

$\begin{array}{lll}-0.72 & 2.86 & 4.37\end{array}$

$-1.18 \quad 2.40 \quad 3.91$

$\begin{array}{lll}-0.76 & 2.82 & 4.33\end{array}$

$\begin{array}{lll}-0.71 & 2.87 & 4.38\end{array}$

$-0.81 \quad 2.77 \quad 4.28$

$\begin{array}{lll}-0.84 & 2.74 & 4.25\end{array}$

$\begin{array}{lll}-0.12 & 3.46 & 4.97\end{array}$

$\begin{array}{lll}-1.22 & 2.36 & 3.87\end{array}$

$\begin{array}{lll}-1.52 & 2.06 \quad 3.57\end{array}$

$\begin{array}{lllll}-0.28^{1} & & -0.28 & 3.30 & 4.81 \\ -1.49^{1} & -1.31 & -1.40 & 2.18 & 3.69\end{array}$

$\begin{array}{llll}917 & -0.59 & -0.25 & -0.23 \\ 388 & -0.70 & & -0.61\end{array}$

$\begin{array}{llll}491 & -0.92 & -0.66 & -1.01\end{array}$

$\begin{array}{llll}608 & -0.64 & -0.49 & -0.68\end{array}$

$-0.36$

3.22

4.73

$-0.66 \quad 2.92$

4.43

4.23

$\begin{array}{lll}-0.60 & 2.98 & 4.49 \\ -0.12 & 3.46 & 4.97\end{array}$

$\begin{array}{llll}367 & -0.06 & 0.03 & -0.22\end{array}$

$-0.12-3.46$

5.01

$\begin{array}{lllll}22 & -1.53 & -1.65 & -1.61^{3} & -1.6\end{array}$

$-0.08 \quad 3.50$
-1.60

$\begin{array}{lll}-1.60 & 1.98 & 3.49\end{array}$

$\begin{array}{llll}916 & -0.33 & -0.14 & -0.39\end{array}$

$\begin{array}{lllll}21 & -0.36 & -0.49^{3} & -0.44 & -0.44^{1}\end{array}$

$\begin{array}{lllll}289 & -0.43 & -0.39 & -0.56^{1} & -0.59\end{array}$

$-0.29$

3.29

4.80

4.62

$\begin{array}{lll}226 & -1.80 & -0.82 \\ 702 & -0.54 & -0.63\end{array}$

$\begin{array}{llll}-1.13 & -1.21 & -1.17^{4} & -1.16\end{array}$

$-0.99$

$-0.82$

$-1.10-1.11$

$-1.16$

$-0.56$

$-0.49$

3.09

4.60

$\begin{array}{llll}223 & -1.16 & -1.02 & -0.89\end{array}$

$-0.58$

3.00

4.51

$-1.29$

$-1.18$

2.40

3.91

$\begin{array}{lll}-0.90 & 2.68 & 4.19 \\ -1.12 & 2.46 & 3.97\end{array}$

$\begin{array}{lll}-1.02 & 2.56 \quad 4.07\end{array}$

$-0.89$

$2.69 \quad 4.20$

$\begin{array}{lllll}287 & -0.55 & -0.48 & -0.67^{1} & -0.47\end{array}$

$\begin{array}{llll}74 & -1.55 & -1.39 & -1.46\end{array}$

$-0.54$

$-1.47$

3.04

4.55

3.62

$387-0.36+-0.42$

$-0.88$

2.70

4.21

$-0.38 \quad 3.20$

4.71

$\begin{array}{llll}367 & -0.58 & -0.46 & -0.55\end{array}$

$\begin{array}{lllll}177 & -0.24 & -0.18 & -0.17^{2} & -0.16\end{array}$

$\begin{array}{llll}21 & -0.39 & -0.34^{3} & -0.45\end{array}$

3.05

4.56

4.91

$-0.43^{\circ}$

3.40

4.71

$176-2.17$ 
TABLE 3.-Oscillator Strengths for Ultraviolet Lines of $\mathrm{Fe} I$-Continued

Wave- Low

length Energy Levels e.p.$$
\AA
$$$$
\mathrm{K}
$$

Volts

\begin{tabular}{|c|c|c|}
\hline 3797.517 & $26106-52431$ & 3.24 \\
\hline 37.97 .950 & $20875-47197$ & 2.59 \\
\hline 3798.513 & $7377-33695$ & 0.91 \\
\hline 3799.550 & $7728-34040$ & 0.96 \\
\hline 3801.682 & $22838-49135$ & 2.83 \\
\hline 3801.804 & $22947-49243$ & 2.84 \\
\hline 3801.975 & $26875-53169$ & 3.33 \\
\hline 3802.283 & $26624-52916$ & 3.30 \\
\hline 3803.220 & $18378-44664$ & 2.28 \\
\hline 3804.013 & $26875-53155$ & 3.33 \\
\hline 3805.345 & $26628-52899$ & 3.30 \\
\hline 3806.203 & $27543-53808$ & 3.41 \\
\hline 3806.699 & $26351-52613$ & 3.27 \\
\hline 3807.539 & $17927-44184$ & 2.22 \\
\hline 3808.286 & $24336-50587$ & 3.02 \\
\hline 3808.731 & $20641-46889$ & 2.56 \\
\hline 3809.043 & $23052-49298$ & 2.86 \\
\hline 3810.759 & $26624-52858$ & 3.30 \\
\hline 3811.050 & $20875-47107$ & 2.59 \\
\hline 3811.892 & $22249-48476$ & 2.76 \\
\hline 3812.966 & $7728-33947$ & 0.96 \\
\hline 3813.059 & $20875-47093$ & 2.59 \\
\hline 3813.638 & $21716-47930$ & 2.69 \\
\hline 3813.891 & $29313-55526$ & 3.63 \\
\hline 3814.525 & $8155-34363$ & 1.01 \\
\hline 3814.785 & $27543-53749$ & 3.41 \\
\hline 3815.843 & $11976-38175$ & 1.48 \\
\hline 3816.342 & $17727-43923$ & 2.20 \\
\hline 3816.908 & $24507-50699$ & 3.04 \\
\hline 3817.650 & $26875-53061$ & 3.33 \\
\hline 3819.497 & $27395-53569$ & 3.40 \\
\hline 3820.427 & $6928-33096$ & 0.86 \\
\hline 3821.181 & $26351-52514$ & 3.27 \\
\hline 3821.836 & $21039-47197$ & 2.61 \\
\hline 3824.076 & $20875-47017$ & 2.59 \\
\hline 3824.306 & $26628-52769$ & 3.30 \\
\hline 3824.446 & $0-26140$ & 0.00 \\
\hline 3825.404 & $18378-44512$ & 2.28 \\
\hline 3825.883 & $7377-33507$ & 0.91 \\
\hline 3826.836 & $21999-48123$ & 2.73 \\
\hline 3827.575 & $21716-47835$ & 2.69 \\
\hline 3827.826 & $12561-38678$ & 1.56 \\
\hline 3828.510 & $22249-48362$ & 2.76 \\
\hline 3829.125 & $29799-55907$ & 3.69 \\
\hline 3829.458 & $26406-52512$ & 3.27 \\
\hline 3829.764 & $20641-46745$ & 2.56 \\
\hline 3830.761 & $21039-47136$ & 2.61 \\
\hline 3830.864 & $21716-47812$ & 2.69 \\
\hline 3833.310 & $20641-46721$ & 2.56 \\
\hline 3834.224 & $7728-33802$ & 0.96 \\
\hline 3836.332 & $26624-52683$ & 3.30 \\
\hline 3837.137 & $21039-47093$ & 2.61 \\
\hline 3839.258 & $24575-50614$ & 3.05 \\
\hline 3839.630 & $31937-57974$ & 3.96 \\
\hline 3840.440 & $7986-34017$ & 0.99 \\
\hline 3841.050 & $12969-38996$ & 1.61 \\
\hline 3842.901 & $20875-46889$ & 2.59 \\
\hline 3842.975 & $20875-46889$ & 2.59 \\
\hline 3843.260 & $24575-50587$ & 3.05 \\
\hline 3845.171 & $19552-45552$ & 2.42 \\
\hline
\end{tabular}

Mult. No.

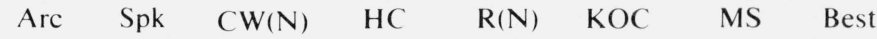

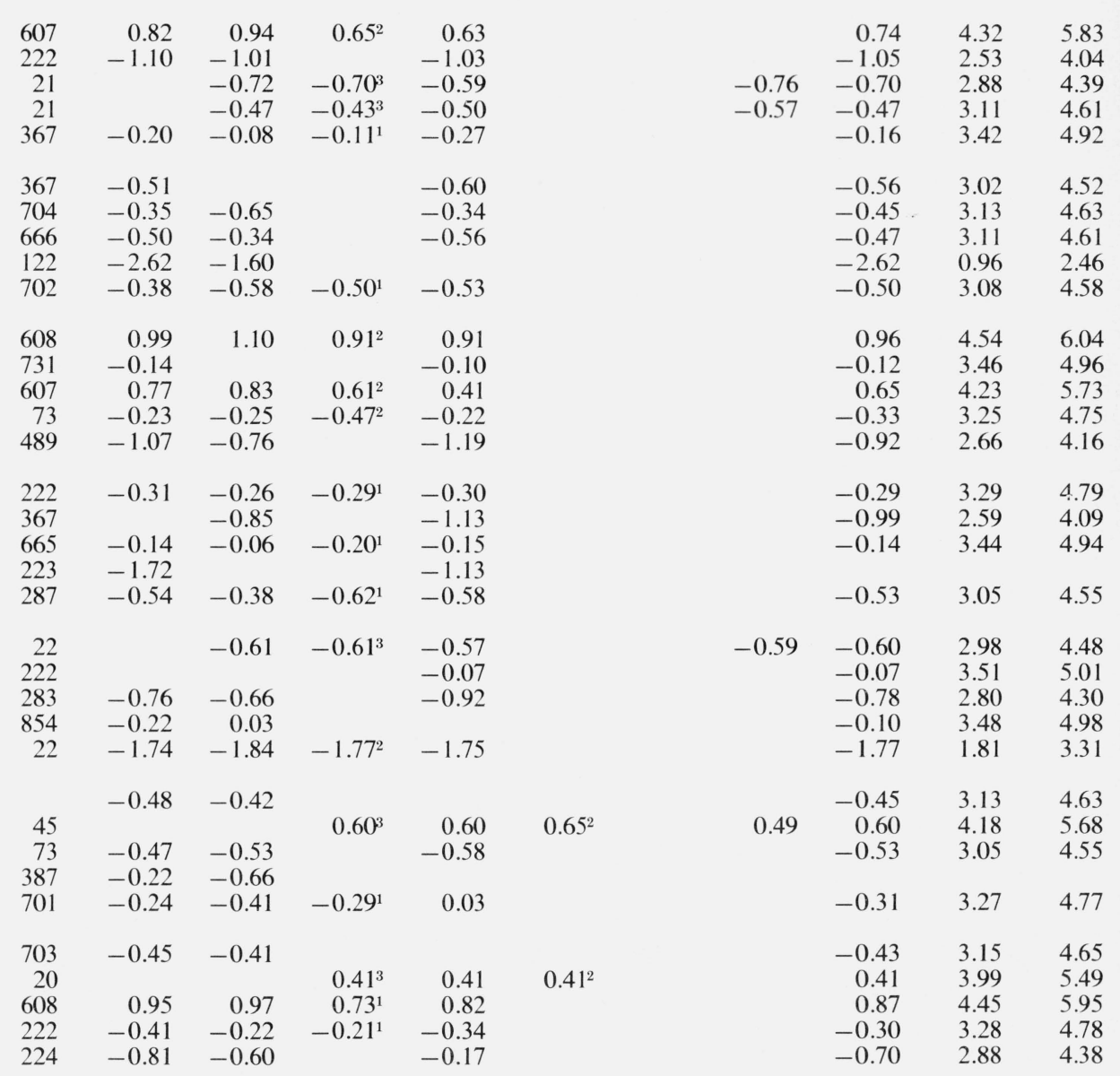

607

4 123

20
283

284

45

287

948
663

221

224

284

221

664

222

529

995

1.52

-1.26
$-1.50 \quad-0.86$

$-1.00-0.68$

$$
\begin{array}{rrr} 
& 0.42 & \\
-1.15^{3} & -1.19 & -1.14^{2} \\
& -1.49 & \\
0.24^{3} & 0.35 & 0.24^{1} \\
& -0.76 &
\end{array}
$$

$\begin{array}{ll}-1.19 & -0.79\end{array}$

$$
0.53
$$

$-0.64-0.58$

$-1.26$

$-0.79$

0.39

$\begin{array}{ll}-1.31 & -1.26\end{array}$

$-0.36$

$-0.40$

$-0.80$

$-0.19$

$-0.14$

$-0.59$

$-0.72$

$\begin{array}{ll}-0.59^{1} & -0.72 \\ -0.14^{2} & -0.14\end{array}$

$-0.02^{3}$

0.04

$0.06^{2}$

$0.56^{1}$

$-1.17 \quad 2.41$

3.91

$\begin{array}{lll}0.26 & 3.84 & 5.34\end{array}$

$-0.81$

2.77

$-0.70$

0.55

2.88

4.13

$-0.67$

$-0.38$

2.91

$-1.28$

2.30

$-0.70$

$-0.15$

0.25
-0.89
0.40

0.35

$0.28^{1}$

0.30

-0.89
0.52

$-0.90^{2}$
$0.30^{2}$

$-0.85$

$\begin{array}{ll}-0.57 & 0.32\end{array}$

$-0.20^{3} \quad-0.15 \quad-0.15^{1}$

$\begin{array}{lll}0.45^{3} & 0.68 & 0.48^{1}\end{array}$

$-1.30$

$-1.47$

0.47
-0.63

$\begin{array}{rrr}0.67 & 0.49^{2} & 0.54 \\ -0.48 & -0.55^{1} & -0.58\end{array}$

$-1.12$

124 
TABLE 3.-Oscillator Strengths for Ultraviolet Lines of Fe I-Continued

Wave-

Energy Levels Low

Volts

$3845.692 \quad 28605-54600 \quad 3.55$

$3846.001 \quad 27167-53161 \quad 3.37$

$\begin{array}{lll}3846.412 & 28820-54811 & 3.57\end{array}$

$3846.802 \quad 26225-52213 \quad 3.25$

$3848.299 \quad 21039-47017 \quad 2.61$

$3849.969 \quad 8155-34122 \quad 1.01$

$3850.819 \quad 7986-33947 \quad 0.99$

$\begin{array}{lll}3852.575 & 17550-43500 & 2.18\end{array}$

$3853.462 \quad 23784-49727 \quad 2.95$

$3854.375 \quad 25900-51837 \quad 3.21$

$\begin{array}{lll}3855.329 & 21999-47930 & 2.73\end{array}$

$3855.846 \quad 26140-52067 \quad 3.24$

3856.373

3858.474

3859.214

$416-26340 \quad 0.05$

$26140-52050 \quad 3.24$

$19390-45295 \quad 2.40$

$3859.913 \quad 0-25900 \quad 0.00$

$3861341 \quad 21716-47606 \quad 2.69$

$3861.600 \quad 26624-52512 \quad 3.30$

$\begin{array}{lll}3863.745 & 21716-47590 & 2.69\end{array}$

$\begin{array}{lll}3864.307 & 20875-46745 \quad 2.59\end{array}$

$\begin{array}{lll}3865.526 & 8155-34017 \quad 1.01\end{array}$

$3867.218 \quad 24336-50187 \quad 3.02$

$\begin{array}{lll}3867.925 & 20875-46721 & 2.59\end{array}$

$\begin{array}{lll}3868.243 & 23784-49628 & 2.95\end{array}$

$\begin{array}{lll}3869.561 & 21999-47835 & 2.73\end{array}$

$\begin{array}{lll}3871.751 & 23784-49604 & 2.95\end{array}$

3872.503

3872.923

3873.762

3874.053

3876.041

3876.670

3878.021

3878.574

3878.676

3878.740

3883.282

3884.361

3885.154

3885.512

3886.284

3887.050

3888.516

3888.825

3889.284

3889.931

3890.390

3890.844

3891.928

3892.302

3892.894

3892.980

3893.316

3893.393

3893.914

3894.005

3894.490

3895.450

3895.658

3897.452

$\begin{array}{ll}7986-33802 & 0.99\end{array}$

$21999-47812 \quad 2.73$

$18378-44184 \quad 2.28$

$8155-33947 \quad 1.01$

$18378-44166 \quad 2.28$

$7728-33507 \quad 0.96$

$704-26479 \quad 0.09$

$19788-45563 \quad 2.45$

$26406-52181 \quad 3.27$

$26225-51969 \quad 3.25$

$21716-47453 \quad 2.69$

$24119-49851 \quad 2.99$

$19552-45282 \quad 2.42$

416-26140 0.05

$7377-33096 \quad 0.91$

$12969-38678 \quad 1.61$

$24336-50043 \quad 3.02$

$\begin{array}{ll}21716-47420 & 2.69\end{array}$

$26340-52040 \quad 3.26$

$26140-51837 \quad 3.24$

$21999-47693 \quad 2.73$

$27543-53230 \quad 3.41$

$28605-54289 \quad 3.55$

$22249-47930 \quad 2.76$

$22838-48516 \quad 2.83$

$23784-49461 \quad 2.95$

$19621-45295 \quad 2.43$

26624-52297 3.30

$25900-51570 \quad 3.21$

$26550-52214 \quad 3.29$

$888-26550 \quad 0.11$

$23784-49434 \quad 2.95$
Mult.
No.

Arc S

Spk $\quad \mathrm{CW}(\mathrm{N})$

$\mathrm{HC} \quad \mathrm{R}(\mathrm{N}) \quad \mathrm{KOC}$

MS Best

$\begin{array}{ll}\log g f \lambda & \log \\ g A / \lambda\end{array}$

771

703

804

224

$\begin{array}{ll}-0.75 & -0.67\end{array}$

$-0.74$

0.00

0.58

0.30

$-1.23$

$-1.23$

20

22
73

$\begin{array}{lllll} & -0.52 & -0.44^{2} & -0.43 & -0.29^{1} \\ -1.20 & -1.18 & -1.24^{2} & -1.26 & \\ -0.44 & -0.47 & -0.58^{2} & -0.76 & \\ -1.18 & -0.85 & & -0.91 & \end{array}$

429
567

283

567

565

175

4

283

663

280

$-1.24-1.10$

$-0.56-0.78$

$-1.25$

$\begin{array}{rr}-0.38 & -0.78 \\ 0.01 & 0.09\end{array}$

$-1.56$

$-0.59$

$\begin{array}{lll}-1.13^{3} & -1.13-1.13^{3}\end{array}$

$0.07^{2} \quad 0.08$

$-1.13^{3}$

$\begin{array}{lllll} & & -0.54^{3} & -0.63 & -0.56^{2} \\ -0.63 & -0.35 & -0.52^{1} & -0.69 & \\ -1.25 & -0.64 & & -0.76 & \\ -0.64 & -0.54 & -0.70^{1} & -0.73 & \end{array}$

$-0.63$

$\begin{array}{llll}-0.64 & -0.54 & -0.70^{1} & -0.73\end{array}$

$19621-45428 \quad 2.43$

$26340-52020 \quad 3.26$
20

488

221

430

284

429

20

284

175

120

12

121
20

4
175

664

$$
-1.98
$$
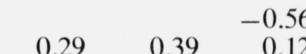

$-1.43$

0.39

$-0.5$

$-0.99$

$-0.93$

$-0.30$

$-1.21^{1}$

$-0.60$

0.20

$-0.49$

$-0.52^{1}-0.66$

$-0.19$

0.05

$-0.15$

$-0.2$

$-1.03$

$-0.62$

$-0.04$

$-0.04$

$-0.54^{3}$

$-0.54$

$-0.82$

$-0.15^{2} \quad-0.02$

$-1.00$

$\begin{array}{llll}-2.32 & -2.37 & -2.20^{1} & -2.34\end{array}$

$-2.16$

$-0.65$

$\begin{array}{lll}-2.20^{1} & -2.34 & \\ & -2.10 & \\ -0.50^{3} & -0.51 & \\ -1.19^{3} & -1.18 & -1.13^{2} \\ & -1.45 & \end{array}$

$-0.80$

$-0.08$

0.11

$0.02^{1}$

$-0.11$

282

430

$-0.54$

$-0.32$

$-0.37^{1}$

$-0.37$

$-0.78$

$-0.34$

20

45

488

280

$-0.97$

$\begin{array}{llll}-1.14 & -0.90^{3} & -0.91 & -0.91^{2}\end{array}$

-0.11
0.00

$-0.13$

$-0.01 \quad 0.02^{1}$

$-1.66$

0.37

564

567

$-1.21$

$-0.85$

$-0.87$

$\begin{array}{llll}-0.61 & -0.45 & -0.62^{1} & -0.62\end{array}$

733

$-0.04$

$-1.59$

0.23

$-0.02^{1}$

$-0.05$

283

$-1.26-1.07$

$-1.45$

567

364

430

$-0.93$

$-1.07$

0.27

0.31

0.20

$-0.76$

$-0.96$

$-0.89$

$\begin{array}{llll}663 & -0.20 & -0.06 & -0.31\end{array}$

$-0.72$

565

4

$-1.83$

$-0.70$

429

$-0.51-1.55-1.47^{3}$

$-1.50$

$-0.71$
$-1.05$

$-0.35-0.93$

$-0.59$

$-0.68$

2.90

2.71

$0.16 \quad 3.75$

0.59

$-1.23$

2.36

$-0.45$

$-1.22$

$-0.57$

$-0.98$

$-0.51$

$-1.17$

$-0.64$

$-1.14$

0.06

$-0.62$

$-0.57$

$-0.55$

$-0.70$

$-0.65$

$-1.98$

$-0.57$

0.22

$-1.08$

$-1.43$

$-0.49$

$-0.12$

$-0.65$ 
Wave-

length Energy Levels Low $K_{K}$

3897.89
3898.01
3899.03
3899.70

3900.519

3902.948

3903.901

3906.481

3906.748

3907.464

3907.937

3908.69

3909.664

3909.830

3910.846

3911.005

3911.699

3913.634

3914.273

3916.733

3917.18

3918.317

3918.418

3918.644

3919.068

3920.260

3920.645

3920.839

3921.270

3922.100

$3922.913 \quad 416-25900 \quad 0.05$

$3925.201 \quad 26550-52020 \quad 3.29$

$3925.646 \quad 22838-48305 \quad 2.83$

$3925.946 \quad 23052-48516 \quad 2.86$

$3926.001 \quad 26140-51604 \quad 3.24$

$3927.922 \quad 888-26340 \quad 0.11$

$3929.114 \quad 22249-47693 \quad 2.76$

$3929.208 \quad 26225-51668 \quad 3.25$

$\begin{array}{lll}3930.298 & 704-26140 & 0.09\end{array}$

$3931.122 \quad 26340-51771 \quad 3.26$

$3932.629 \quad 26406-51828 \quad 3.27$

$3933.606 \quad 24772-50187 \quad 3.07$

$\begin{array}{lll}3935.306 & 22947-48351 & 2.84\end{array}$

$\begin{array}{lll}3935.814 & 22838-48239 & 2.83\end{array}$

3936.772

3937.331

3940.044

3940.880

3941.283

3942.442

$\begin{array}{lll}3943.341 & 17727-43079 & 2.20\end{array}$

$3944.748 \quad 22947-48290 \quad 2.84$

$3944.892 \quad 24119-49461 \quad 2.99$

$3945.119 \quad 22249-47590 \quad 2.76$

$3947.002 \quad 25900-51229 \quad 3.21$

$\begin{array}{lll}3947.391 & 19351-44677 & 2.40\end{array}$

$\begin{array}{lll}3947.533 & 22838-48163 & 2.83\end{array}$

$3948.105 \quad 26140-51462 \quad 3.24$

$\begin{array}{lll}3948.778 & 26351-51668 & 3.27\end{array}$

3949.156
Mult.

No.

$\begin{array}{llllll}\text { Arc Spk } & \mathrm{CW}(\mathrm{N}) \quad \mathrm{HC} & \mathrm{R}(\mathrm{N}) & \mathrm{KOC} & \mathrm{MS} \text { Best }\end{array}$ $\log \operatorname{gf} \lambda$

$\log$

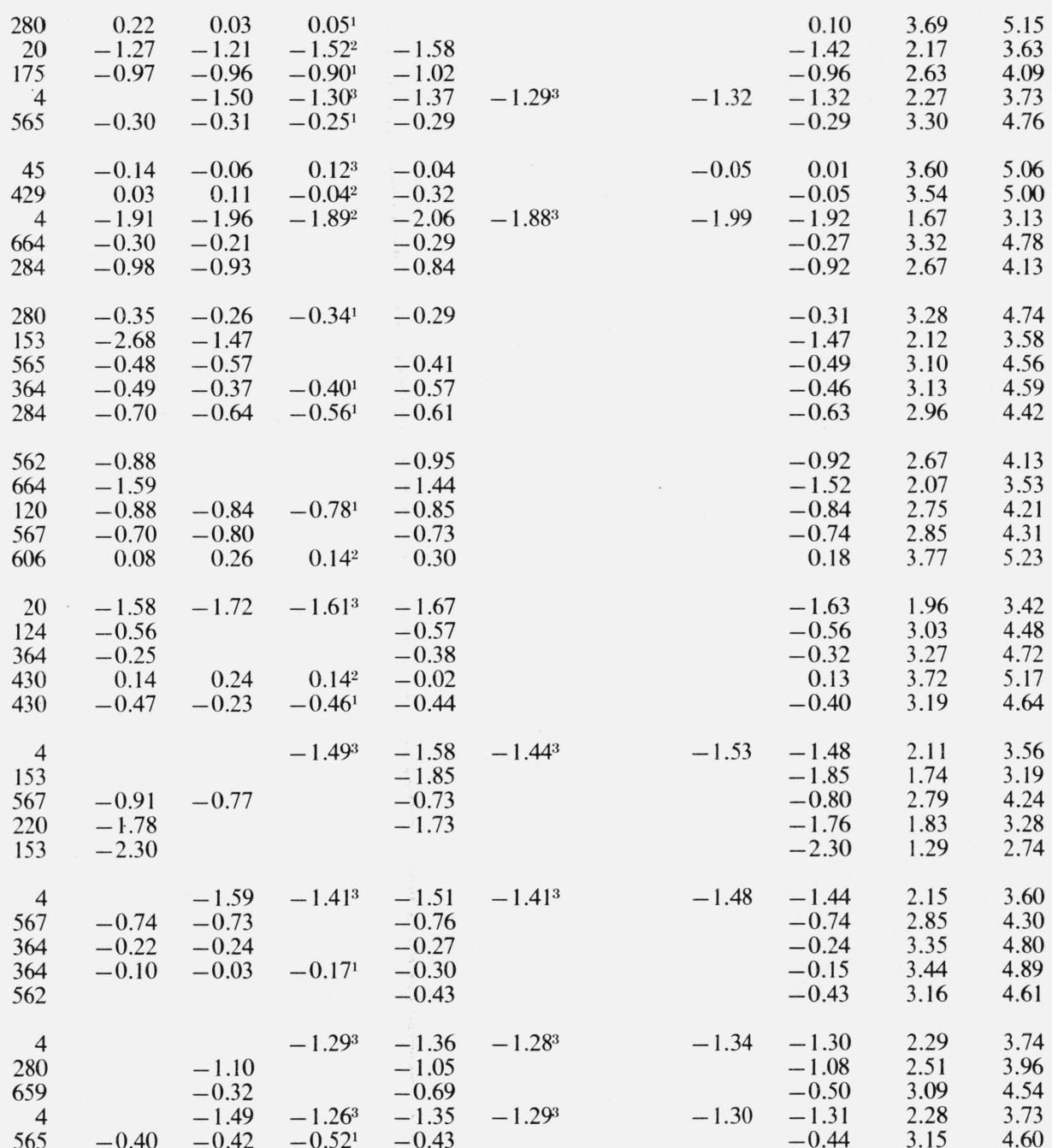

$\begin{array}{lllll}652 & -0.03 & -0.04 & -0.47^{1} & -0.46\end{array}$

$\begin{array}{lllll}488 & -0.37 & -0.11 & -0.42^{1} & -0.43\end{array}$

$\begin{array}{lllll}362 & -0.85 & -0.85 & -1.01^{1} & -0.81\end{array}$

$\begin{array}{lllll}362 & -0.11 & -0.07 & -0.14^{2} & -0.16\end{array}$

$-1.91$

$-0.33$

3.26

$-0.88$

2.71

$-1.91$

3.48

4.71

4.16

4.92

278

$\begin{array}{llll}-0.60 & -0.58 & -0.631 & -0.64\end{array}$

$-0.61$

2.99

4.43

$-1.27-0.81$

$-1.99$

$-2.12$

$\begin{array}{rr}-0.63-0.64 \\ -1.992 & -1.14\end{array}$

$\begin{array}{ll}-1.99^{2} & -2.08\end{array}$

$\begin{array}{ll}-0.52^{1} & -0.49\end{array}$

$\begin{array}{llll}-0.27 & -0.15 & -0.19^{2} & -0.41\end{array}$

$\begin{array}{ll}-2.03 & 1.57 \\ -0.52 & 3.08\end{array}$

3.01

364

$-1.40$

$\begin{array}{lll}-1.41 & -1.34^{1} & -1.29\end{array}$

361

$-1.26$

430

$-0.61$

$-0.50-0.66^{1}$

$-1.11$

561

$\begin{array}{lll}-0.60 & -0.80^{1} \quad-0.71\end{array}$

$\begin{array}{lll}-0.36 & -0.24^{1} \quad-0.45\end{array}$

$-0.24$

3.36

4.80

$\begin{array}{lll}-1.36 & 2.24 & 3.68\end{array}$

$\begin{array}{lll}-1.18 & 2.42 & 3.86\end{array}$

$\begin{array}{lll}-0.62 & 2.98 & 4.42\end{array}$

$\begin{array}{lll}-0.69 & 2.91 \quad 4.35\end{array}$

$\begin{array}{lll}-0.33 & 3.27 & 4.71\end{array}$

$\begin{array}{rrrrr}153 & -2.30 & & & -1.85 \\ 361 & -0.38 & -0.24 & -0.37^{1} & -0.40 \\ 562 & 0.09 & -0.06 & 0.13^{1} & 0.00 \\ 604 & 0.38 & 0.49 & 0.38^{2} & 0.29 \\ 730 & -0.91 & & & -0.78\end{array}$

$-0.35 \quad 3.25$

$0.04 \quad 3.64$

4.68

$0.38 \quad 3.98$

5.07

$\begin{array}{rr}-0.84 & 2.76\end{array}$ 
TABLE 3.-Oscillator Strengths for Ultraviolet Lines of Fe 1-Continued

Wave-

Wave- Low
length Energy Levels e.p.

3949.956

3951.164

3951.638

3952.604

3952.702

$\begin{array}{lll}3953.155 & 24339-49628 \quad 3.02\end{array}$

$3953.512 \quad 28605-53892 \quad 3.55$

$3953.861 \quad 22838-48123 \quad 2.83$

$3954.715 \quad 26351-51630 \quad 3.27$

$3955.352 \quad 26479-51755 \quad 3.28$

3955.956

3956.457

3956.680

3957.027

3957.620

3960.284

3961.147

3962.353

3963.108

3963.438

3964.517

3965.511

3966.064

3966.532

3966.630

3966.824

3967.423

3967.964

3969.260

3969.628

3970.391

3971.325

3971.820

3972.918

3973.655

3974.397

3974.764

3975.210

3975.842

3976.390

3976.615

3976.865

3977.744

3978.464

3979.630

3980.650

3981.104

3981.774

3983.350

3983.959

3984.930

3985.322

3985.393

3986.176

3989.006

3989.859

3990.377

3992.395

3994.117

3995.199
$17550-42860 \quad 2.18$

2.18
2.27

$23052-48351 \quad 2.86$

$21716-47008 \quad 2.69$

$22947-48239 \quad 2.84$

$24772-50043 \quad 3.07$

$26106-51374 \quad 3.24$

$21716-46982 \quad 2.69$

$26340-51604 \quad 3.26$

$26479-51740 \quad 3.28$

$29357-54600 \quad 3.64$

$23052-48290 \quad 2.86$

$26340-51570 \quad 3.26$

$26479-51705 \quad 3.28$

$26406-51630 \quad 3.27$

$22947-48163 \quad 2.84$

$26140-51351 \quad 3.24$

$12969-38175 \quad 1.61$

$26624-51828 \quad 3.30$

$25900-51103 \quad 3.21$

$26624-51826 \quad 3.30$

$26628-51826 \quad 3.30$

$26140-51335 \quad 3.24$

$11976-37163 \quad 1.48$

$26225-51409 \quad 3.25$

$24772-49951 \quad 3.07$

$21716-46889 \quad 2.69$

$22249-47420 \quad 2.76$

$28820-53983 \quad 3.57$

$28605-53763 \quad 3.55$

$26140-51294 \quad 3.24$

$17927-43079 \quad 2.22$

$19913-45061 \quad 2.47$

$31307-56452 \quad 3.88$

$24336-49477 \quad 3.02$

$27543-52683 \quad 3.41$

$24339-49477 \quad 3.02$

$17727-42860 \quad 2.20$

$22838-47967 \quad 2.83$

$26340-51461 \quad 3.26$

$19562-44677 \quad 2.42$

$19552-44664 \quad 2.42$

$21999-47107 \quad 2.73$

$24336-49433 \quad 3.02$

$21999-47093 \quad 2.73$

$26479-51567 \quad 3.28$

$20641-45726 \quad 2.56$

$26624-51708 \quad 3.30$

$26225-51305 \quad 3.25$

$28820-53882 \quad 3.57$

$28605-53661 \quad 3.55$

$24575-49628 \quad 3.05$

$26628-51668 \quad 3.30$

$24575-49604 \quad 3.05$

$26351-51374 \quad 3.27$

Mult. Arc Spk CW(N) $\mathrm{HC} \quad \mathrm{R}(\mathrm{N}) \quad \mathrm{KOC}$ MS Best $\log \operatorname{gf} \lambda$

$\log$ No.

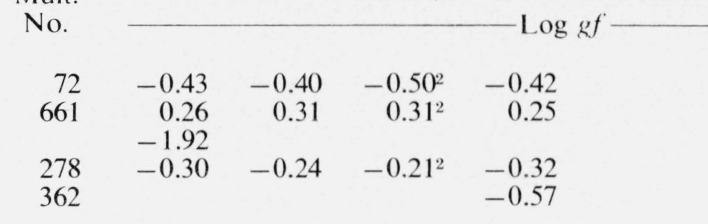

\begin{tabular}{rrrrr} 
No. & & & & Log $g f-$ \\
\cline { 2 - 5 } 72 & -0.43 & -0.40 & $-0.50^{2}$ & -0.42 \\
661 & 0.26 & 0.31 & $0.31^{2}$ & 0.25 \\
& -1.92 & & & \\
278 & -0.30 & -0.24 & $-0.21^{2}$ & -0.32 \\
362 & & & & -0.57
\end{tabular}

$-0.45$

3.15

0.29

$-1.92$

$-0.26$

$-0.57$

$\begin{array}{lllll}430 & -0.34 & -0.25 & -0.38^{1} & -0.44\end{array}$

$\begin{array}{lllll}362 & -1.17 & -1.04 & -1.23^{1} & -1.06\end{array}$

$\begin{array}{lllll}606 & -1.53 & -0.85 & & -1.21 \\ 562 & -0.37 & -0.75 & -0.41^{1} & -0.49\end{array}$

$\begin{array}{lllll}562 & -0.37 & -0.75 & -0.41^{1} & -0.49\end{array}$

$\begin{array}{lllll}488 & -0.66 & -0.56 & -0.68^{1} & -0.68\end{array}$

$\begin{array}{lllll}604 & 0.41 & 0.49 & 0.41^{1} & 0.54\end{array}$

$\begin{array}{lllll}278 & 0.51 & 0.41 & 0.33^{1} & 0.31\end{array}$

$\begin{array}{lllr}562 & -0.08 & -0.26 & 0.00 \\ 564 & -1.47 & -1.03 & -1.27\end{array}$

$-0.35$

$-1.48$

$-1.12$

$-0.50$

3.89

1.68

3.34

3.03

5.32

3.11

4.77

4.46

3.25

4.68

$-1.27$

$\begin{array}{lllll}913 & -0.55 & -0.58 & -0.61^{2} & -0.69 \\ 361 & -0.89 & -0.90 & -1.00^{2} & -0.83 \\ 566 & -0.99 & -1.04 & -1.01^{1} & -0.89\end{array}$

$\begin{array}{lllll}562 & -0.08 & -0.29 & -0.09^{1} & -0.15\end{array}$

$654-1.50$

$\begin{array}{lllll}361 & -0.71 & -0.68 & -0.80^{1} & -0.65\end{array}$

$\begin{array}{lllll}361 & -0.71 & -0.68 & -0.80^{1} & -0.65 \\ 565 & -0.72 & -0.83 & & -0.78\end{array}$

$\begin{array}{rrrrr}45 & -1.02 & -1.16 & -0.99^{2} & -1.23 \\ 652 & & & & -0.86\end{array}$

$\begin{array}{llll}562 & 0.13 & 0.34^{2} & 0.34\end{array}$

$$
\begin{aligned}
& 2.12 \\
& 2.48
\end{aligned}
$$

3.55
3.91

3.10

4.53

$-0.6$

\subsection{6}

0.46
0.39

4.06

3.99

4.39

$-0.11$

3.49
2.23

5.49

5.42

$-1.37$

4.92
3.66

$-0.61 \quad 2.99$

$-0.92$

$-0.98$

$-0.15$

2.68

4.42

4.11

$-1.50$

2.62
3.45

4.05

4.88

$-0.71$

$-0.78$

2.10

3.53

659

604

561

43
657

488

27

281

803
769

0.25

0.15

$\begin{array}{rrrr}0.25 & 0.36 & 0.34^{2} & -0.42 \\ -0.23 & -0.38 & -0.16^{1} & -1.03\end{array}$

$-0.13$

$-0.17$

$\begin{array}{rr}-0.02^{3} & -1.03 \\ & 0.08\end{array}$

$-0.31$

$0.03^{1}$

$-0.26$

$-1.86$

$-0.26^{1}-0.28$

$-0.13^{2}-0.14$

$-1.94$

$-0.87-1.36$

$\begin{array}{llll}-0.25 & -0.29 & -0.31^{1} & -0.30\end{array}$

$-1.08$

$-0.86$

2.89

4.32

\subsection{2}

2.52

0.29

3.89

3.95

4.17

0.15

0.32

3.75

$-0.26$

3.92

0.00

-0.02
-0.30

3.34

3.58
3.30

$-0.26$

3.34

$-0.14 \quad 3.46$

$-1.90$

1.70

$-0.29 \quad 3.31$

4.74

$564-1.1$

$-1.08$

$-0.71$

$-1.86$

$-1.92$

$-0.81 \quad-0.81$

$487-1.30$

729

431

36

561

0.06

$\begin{array}{rr}0.06 & -0.58\end{array}$

$-0.31$

$-0.35$

$-1.42$

$-1.10$

2.50

$-1.90$

$-1.92$

$-0.81$

1.70

$-1.36$

2.79

2.24

3.13

3.11

4.22

0.02

3.62

$-0.63$

$-0.28$

$-1.28$

$-1.06$

3.32

2.32 
TABLE 3.-Oscillator Strengths for Ultraviolet Lines of Fe I-Continued

Wave-

length Energy Levels

3995.986

3996.261

3996.779

3996.968

3997.395

3998.055

4000.266

4000.460

4001.663

4002.665

4003.764

4004.832

4004.976

4005.244

4006.159

4006.3

4006.631

4006.768

4007.274

4009.715

4010.180

4010.770

4011.412

4011.710

4012.160

4013.641

4013.798

4013.822

4014.280

4014.534

4016.429

4017.093

4017.152

4018.282

4019.050

4020.490

4021.622

4021.870

4022.212

4022.450

4022.744

4024.109

4024.735

4029.640

4030.186

4030.499

4031.243

4031.727

4031.965

4032.469

4032.629

4033.190

4036.370

4037.725

4038.622

4039.940

4040.650

4041.288

4041.911

4043.901
$21999-47017 \quad 2.73$

$24119-49135$

$33507-58520$

$29799-5481$

$21999-47008$

$21716-46721 \quad 2.69$

$26340-51331 \quad 3.26$

$24119-49109 \quad 2.99$

$17550-42533 \quad 2.18$

$23245-48221 \quad 2.88$

$27543-52512 \quad 3.41$

$26106-51069 \quad 3.24$

$24336-49298 \quad 3.02$

$12561-37521 \quad 1.56$

$26340-51294 \quad 3.26$

$26351-51305 \quad 3.27$

$25092-50043 \quad 3.11$

$23270-48221 \quad 2.88$

$22249-47197 \quad 2.76$

$17927-42860 \quad 2.22$

$29372-54301 \quad 3.64$

$23111-48037 \quad 2.86$

$20641-45563 \quad 2.56$

$19757-44677 \quad 2.45$

26106-51023 3.24

$25900-50808 \quad 3.21$

$24336-49243 \quad 3.02$

$24336-49243 \quad 3.02$

$24339-49243 \quad 3.02$

$28820-53722 \quad 3.57$

$26479-51370 \quad 3.28$

$22249-47136 \quad 2.76$

$24575-49461 \quad 3.05$

$26340-51219 \quad 3.26$

$21039-45914 \quad 2.61$

$29372-54237 \quad 3.64$

$26140-50999 \quad 3.24$

$22249-47107 \quad 2.76$

$22838-47693 \quad 2.83$

$19390-44244 \quad 2.40$

$26479-51331 \quad 3.28$

$22249-47093 \quad 2.76$

$26140-50980 \quad 3.24$

$26340-51149 \quad 3.26$

$17727-42533 \quad 2.20$

$25900-50704 \quad 3.21$

$24336-49135 \quad 3.02$

$24339-49135 \quad 3.02$

$26406-51201 \quad 3.27$

$23245-48037 \quad 2.88$

$11976-36767 \quad 1.48$

$20641-45428 \quad 2.56$

$22249-47017 \quad 2.76$

$18378-43138 \quad 2.28$

$26628-51381 \quad 3.30$

$21999-46745 \quad 2.73$

$26624-51365 \quad 3.30$

$26628-51365 \quad 3.30$

$26628-51361 \quad 3.30$

$21999-46721 \quad 2.73$
Arc Spk $\mathrm{CW}(\mathrm{N})$ HC $\mathrm{R}(\mathrm{N})$ KOC MS Best

Mult.

No.

Log $g f$

$\begin{array}{rrrrrr}279 & -0.58 & -0.65 & -0.66^{2} & -0.78 & \\ 561 & -1.55 & & -1.78^{1} & & \\ 1074 & -1.12 & & -0.61^{1} & & \\ 945 & -0.25 & -0.16 & -0.22^{2} & -0.21 & \\ 278 & 0.45 & 0.36 & 0.38^{3} & 0.32 & \\ 276 & -0.01 & -0.12 & -0.04^{3} & -0.04 & \\ 556 & -0.83 & & -0.87^{1} & -1.08 & \\ 426 & -0.66 & -0.68 & -0.80^{2} & -0.78 & \\ 72 & -1.10 & -1.12 & -1.09^{2} & -1.05 & \\ 320 & -1.84 & & -1.67^{2} & -1.54 & \\ 728 & -0.59 & -0.48 & -0.51^{2} & -0.55 & \\ 601 & & & -0.47^{1} & & \\ 486 & & & -0.76^{1} & -0.85 & \\ 43 & -0.11 & -0.02 & -0.07^{3} & -0.10 & -0.12^{1} \\ 564 & & & -1.56^{1} & & \\ 603 & & & & & \end{array}$

488

320

277

$-0.15$

$-0.43^{1} \quad-0.58$

$\begin{array}{lll}-0.97 & -0.99^{1} & -1.22\end{array}$

$\begin{array}{llll}-0.44 & -0.44 & -0.45^{2} & -0.46\end{array}$

$-0.39$

$-0.42$

$-0.44^{3}$

$-0.43$

915

320

218

153

601

$-1.42$

$-2.03$

$-1.291$

$-1.55$

$\begin{array}{rrrr}-1.40 & -1.40 & -1.54^{1} & -1.50 \\ -1.77 & & -1.85^{1} & -1.91\end{array}$

$-1.85$

$-1.59$

557
485

485
486

426

802

$-0.89-0.98$

$-1.02$

$-0.95$

$-0.71^{1}$

$\begin{array}{lll}-1.32 & -0.71^{1}-0.68\end{array}$

0.38

$-1.14$

$0.58^{3}$

$-1.38$

560
279

$\begin{array}{llll}-0.66 & -0.89 & -0.66^{2} & -0.75\end{array}$

527

560

219

91

557
278

$-1.74$

-0.89
-0.11
-0.78

$-0.17^{2}$

-0.78
-1.59

-0.63
-1.80

$-0.22$

$-1.88$

1.59

$-1.80$

$-1.74$

0.08

$-1.20$

$-1.17$

$-1.66$

0.06

$-2.22^{1}$

$-2.21$

$-1.17^{1}$

$-1.33$

$-1.10$

$-1.16$

$-1.35^{2}$

$-0.04$

$-0.33$

$\begin{array}{llll}-0.04 & -0.33 & -0.06^{2} & -0.10 \\ -0.29 & -0.65 & -0.42^{2} & -0.47\end{array}$

$-1.51$

$-1.51$

$-0.42^{2}$

$-0.05-0.20=0.08^{2}-0.09$

560

486

427

655

320

$-1.51$

$-0.20$

0.08

0.09
-1.58

$-1.49$

$-0.21$

$-1.42$

$-0.26-0.30^{2}$

$-0.33$

$\begin{array}{lll}-1.11 & -1.24^{1} \quad-1.46\end{array}$

$\begin{array}{lllll}44 & -1.74 & -1.89 & -1.74^{2} & -1.85\end{array}$

$\begin{array}{llll}218 & -1.64 & -1.72^{2} & -1.69\end{array}$

279

118

$-2.10$

$-2.03$

$-1.55$

$-1.95^{1}$

$-0.99^{2}$

$276-1.75$

-0.35
-0.72

$-1.54^{2}-1.59$

$\begin{array}{ll}-0.30^{2} & 0.04\end{array}$

$-0.74^{2}-0.92$

$\begin{array}{ll}-1.25^{1} & -1.25 \\ -0.56^{2} & -0.70\end{array}$

602
276
Log $\log g f \lambda \quad g A / \lambda$

$\begin{array}{rll}-0.67 & 2.93 & 4.35 \\ -1.78 & 1.82 & 3.24 \\ -0.61 & 2.99 & 4.41 \\ -0.21 & 3.39 & 4.81 \\ 0.38 & 3.98 & 5.40 \\ & & \\ -0.05 & 3.55 & 4.97 \\ -0.98 & 2.62 & 4.04 \\ -0.74 & 2.86 & 4.28 \\ -1.09 & 2.51 & 3.93 \\ -1.68 & 1.92 & 3.34 \\ -0.53 & 3.07 & 4.49 \\ -0.47 & 3.13 & 4.55 \\ -0.80 & 2.80 & 4.22 \\ -0.09 & 3.51 & 4.93 \\ -1.56 & 2.04 & 3.46\end{array}$

$\begin{array}{lll}-0.18 & 3.42 & 4.84\end{array}$

$\begin{array}{lll}-0.44 & 3.16 & 4.58\end{array}$

$\begin{array}{lll}-1.06 & 2.54 & 3.96\end{array}$

$\begin{array}{lll}-0.45 & 3.15 & 4.57\end{array}$

$-0.43 \quad 3.17$

$-1.42 \quad 2.18$

$-1.46$

2.14

$-1.84 \quad 1.76$

$-1.64 \quad 1.96$

$-0.96 \quad 2.64$

$\begin{array}{ll}-1.07 & 2.53\end{array}$

$-0.70 \quad 2.90$

$\begin{array}{ll}-1.30 & 2.30\end{array}$

$0.54 \quad 4.14$

$-0.72 \quad 2.88$

$\begin{array}{ll}-1.09 & 2.51 \\ -0.17 & 3.43\end{array}$

$-0.17 \quad 3.43$

$\begin{array}{ll}-0.70 & 2.90 \\ -1.75 & 1.85\end{array}$

$-1.06 \quad 2.54$

$-1.14 \quad 2.46$

0.12
-1.70

$-1.70 \quad 1.90$
-2.27

$-2.27$

1.33

$-1.20$

$-1.28$

2.40

$-0.12$

$-0.45$

$-1.50$

3.48

3.16

0.02

$-1.50$

$-1.44$

$-0.28$

$-1.27$

$-1.79 \quad 1.82$

$-1.69$

$-2.02$

$-0.99 \quad 2.62$

$-1.60 \quad 2.01$
-0.27

$-0.27 \quad 3.34$

$\begin{array}{ll}-0.79 & 2.82 \\ - & .25\end{array}$

$-1.25 \quad 2.36$

$\begin{array}{ll}-0.61 & 3.00\end{array}$

4.58 
TABLE 3.-Oscillator Strengths for Ultraviolet Lines of Fe I-Continued

Wave-

length Energy Levels e.p.

K Volts

Mult. Arc Spk $\mathrm{CW}(\mathrm{N})$ HC $\mathrm{R}(\mathrm{N})$ KOC MS Best

$\log$

\begin{tabular}{|c|c|c|c|c|c|c|c|c|c|c|c|c|}
\hline 4043.993 & $26140-50861$ & 3.24 & 559 & & -0.37 & $-0.63^{1}$ & & & & -0.50 & 3.11 & 4.50 \\
\hline 4044.612 & $22838-47556$ & 2.83 & 359 & -0.30 & 0.14 & $-0.17^{2}$ & -0.33 & $-0.30^{1}$ & & -0.19 & 3.42 & 4.81 \\
\hline 4045.815 & $11976-36686$ & 1.48 & 43 & & & $0.68^{3}$ & 0.65 & & 0.62 & 0.66 & 4.27 & 5.66 \\
\hline 4046.629 & $24772-49477$ & 3.07 & 487 & & & & -1.18 & & & -1.18 & 2.43 & 3.82 \\
\hline 4047.315 & $18378-43079$ & 2.28 & 117 & -1.68 & & $-1.84^{1}$ & -1.88 & & & -1.80 & 1.81 & 3.20 \\
\hline 4049.331 & $20875-45563$ & 2.59 & 218 & -1.31 & -1.34 & $-1.35^{2}$ & -1.39 & & & -1.35 & 2.26 & 3.65 \\
\hline 4051.923 & $27395-52067$ & 3.40 & 700 & -0.80 & -0.91 & $-0.68^{2}$ & -0.82 & & & -0.78 & 2.83 & 4.22 \\
\hline 4052.312 & $27167-51837$ & 3.37 & 700 & -0.93 & & $-0.89^{1}$ & -0.96 & & & -0.93 & 2.68 & 4.07 \\
\hline 4052.466 & $26479-51149$ & 3.28 & 563 & -0.83 & & $-1.04^{1}$ & -1.06 & & & -0.98 & 2.63 & 4.02 \\
\hline 4052.664 & $24575-49243$ & 3.05 & 524 & & -1.05 & $-1.14^{1}$ & -1.36 & & & -1.18 & 2.43 & 3.82 \\
\hline 4052.724 & $26140-50808$ & 3.24 & 557 & & & $-1.16^{1}$ & -1.07 & & & -1.11 & 2.50 & 3.89 \\
\hline 4053.820 & $24772-49433$ & 3.07 & 485 & -1.16 & & $-1.47^{1}$ & -1.34 & & & -1.32 & 2.29 & 3.68 \\
\hline 4054.180 & $26340-50999$ & 3.26 & 557 & -1.28 & & $-1.17^{1}$ & -1.24 & & & -1.23 & 2.38 & 3.77 \\
\hline 4054.833 & $27395-52050$ & 3.40 & 698 & & & $-0.15^{1}$ & -0.42 & & & -0.28 & 3.33 & 4.72 \\
\hline 4054.883 & $27560-52214$ & 3.42 & 698 & & -0.33 & $-0.21^{1}$ & -0.40 & & & -0.31 & 3.30 & 4.69 \\
\hline 4055.038 & $20641-45295$ & 2.56 & 218 & -0.84 & -0.84 & $-0.86^{1}$ & -0.54 & & & -0.77 & 2.84 & 4.23 \\
\hline 4055.980 & $29357-54005$ & 3.64 & 914 & -1.81 & & $-1.60^{1}$ & -1.59 & & & -1.67 & 1.94 & 3.33 \\
\hline 4056.530 & $23111-47756$ & 2.86 & 320 & -2.10 & & $-1.82^{1}$ & & & & -1.96 & 1.65 & 3.04 \\
\hline 4057.346 & $22249-46889$ & 2.76 & 277 & -1.00 & & $-1.04^{2}$ & -1.09 & & & -1.04 & 2.57 & 3.96 \\
\hline 4057.654 & $27543-52181$ & 3.41 & 729 & -2.01 & & $-1.69^{1}$ & & & & -1.85 & 1.76 & 3.15 \\
\hline 4058.227 & $25900-50534$ & 3.21 & 558 & -0.37 & -0.50 & $-0.28^{2}$ & 0.01 & & & -0.38 & 3.23 & 4.62 \\
\hline 4058.756 & $19552-44184$ & 2.42 & 120 & -1.18 & -1.31 & $-1.19^{2}$ & -1.18 & & & -1.21 & 2.40 & 3.79 \\
\hline 4059.726 & $28605-53230$ & 3.55 & 767 & -0.50 & -0.52 & $-0.52^{2}$ & -0.52 & & & -0.52 & 3.09 & 4.48 \\
\hline 4062.444 & $22947-47556$ & 2.84 & 359 & 0.02 & 0.02 & $0.05^{3}$ & 0.02 & $0.05^{1}$ & & 0.04 & 3.65 & 5.04 \\
\hline 4063.286 & $27167-51771$ & 3.37 & 698 & -0.06 & & $0.34^{1}$ & 0.03 & & & 0.10 & 3.71 & 5.10 \\
\hline 4063.596 & $12561-37163$ & 1.56 & 43 & & & $0.43^{2}$ & 0.56 & $0.42^{2}$ & 0.41 & 0.44 & 4.05 & 5.44 \\
\hline 4064.450 & $12561-37158$ & 1.56 & 44 & -2.27 & & $-2.47^{1}$ & -2.27 & & & -2.34 & 1.27 & 2.66 \\
\hline 4065.392 & $27666-52257$ & 3.43 & 698 & -0.50 & -0.67 & $-0.70^{2}$ & -0.62 & & & -0.64 & 2.97 & 4.36 \\
\hline 4066.590 & $24119-48703$ & 2.99 & 424 & -0.73 & -0.61 & $-0.51^{1}$ & -0.82 & & & -0.67 & 2.94 & 4.33 \\
\hline 4066.979 & $22838-47420$ & 2.83 & 358 & -0.25 & -0.20 & $-0.19^{2}$ & -0.30 & & & -0.23 & 3.38 & 4.77 \\
\hline 4067.274 & $20641-45221$ & 2.56 & 217 & -0.52 & -0.65 & $-0.37^{1}$ & -0.53 & & & -0.52 & 3.09 & 4.48 \\
\hline 4067.984 & $25900-50475$ & 3.21 & 559 & 0.30 & 0.03 & $0.29^{2}$ & 0.31 & & & 0.24 & 3.85 & 5.24 \\
\hline 4069.080 & $26479-51048$ & 3.28 & 557 & -1.25 & & $-1.13^{2}$ & -1.10 & & & -1.15 & 2.46 & 3.85 \\
\hline 4070.766 & $26140-50699$ & 3.24 & 558 & -0.03 & -0.33 & $0.01^{2}$ & & & & -0.08 & 3.53 & 4.91 \\
\hline 4071.520 & $20875-45428$ & 2.59 & 218 & & & $-0.88^{1}$ & & & & -0.88 & 2.73 & 4.11 \\
\hline 4071.740 & $12969-37521$ & 1.61 & 43 & & & $0.40^{3}$ & 0.44 & $0.46^{2}$ & 0.35 & 0.42 & 4.03 & 5.41 \\
\hline 4072.518 & $27666-52214$ & 3.43 & 698 & -0.45 & -0.63 & $-0.43^{1}$ & -0.56 & & & -0.52 & 3.09 & 4.47 \\
\hline 4073.760 & $26340-50880$ & 3.26 & 558 & -0.08 & -0.35 & $-0.14^{2}$ & -0.02 & & & -0.15 & 3.46 & 4.84 \\
\hline 4074.789 & $24575-49109$ & 3.05 & 524 & -0.14 & -0.17 & $-0.14^{2}$ & -0.24 & & & -0.17 & 3.44 & 4.82 \\
\hline 4076.232 & $24772-49298$ & 3.07 & 486 & -1.13 & -1.20 & $-0.94^{1}$ & -1.10 & & & -1.09 & 2.52 & 3.90 \\
\hline 4076.498 & $21039-45563$ & 2.61 & 218 & & & $-0.90^{1}$ & -1.14 & & & -1.02 & 2.59 & 3.97 \\
\hline 4076.636 & $25900-50423$ & 3.21 & 558 & 0.17 & 0.06 & $0.24^{2}$ & 0.27 & & & 0.18 & 3.79 & 5.17 \\
\hline 4076.810 & $26340-50862$ & 3.26 & 557 & & & $-0.48^{1}$ & -0.59 & & & -0.54 & 3.07 & 4.45 \\
\hline 4076.884 & $26340-50861$ & 3.26 & 559 & & & $-1.42^{1}$ & & & & -1.42 & 2.19 & 3.57 \\
\hline 4078.356 & $21039-45552$ & 2.61 & 217 & -0.59 & -0.72 & $-0.61^{2}$ & -0.58 & & & -0.62 & 2.99 & 4.37 \\
\hline 4078.822 & $29372-53882$ & 3.64 & & -1.88 & & $-1.45^{1}$ & & & & & & \\
\hline 4079.186 & $27560-52067$ & 3.42 & 700 & -0.81 & & $-0.85^{1}$ & & & & -0.83 & 2.78 & 4.16 \\
\hline 4079.841 & $23052-47556$ & 2.86 & 359 & -0.39 & -0.51 & $-0.52^{2}$ & -0.43 & $-0.48^{1}$ & & -0.48 & 3.13 & 4.51 \\
\hline 4080.226 & $26479-50981$ & 3.28 & 558 & -0.33 & -0.66 & $-0.34^{1}$ & -0.43 & & & -0.44 & 3.17 & 4.55 \\
\hline 4080.886 & $26550-51048$ & 3.29 & 557 & -0.92 & & $-1.06^{2}$ & -0.98 & & & -1.00 & 2.61 & 3.99 \\
\hline 4082.125 & $27560-52050$ & 3.42 & 698 & -0.69 & & $-0.70^{1}$ & -0.81 & & & -0.73 & 2.88 & 4.26 \\
\hline 4082.432 & $29320-53808$ & 3.63 & 906 & -0.90 & & $-0.78^{1}$ & -0.88 & & & -0.85 & 2.76 & 4.14 \\
\hline 4083.554 & $18378-42860$ & 2.28 & 117 & -1.54 & & $-1.59^{1}$ & -1.60 & & & -1.58 & 2.03 & 3.41 \\
\hline 4083.780 & $27560-52040$ & 3.42 & 697 & -0.61 & & $-0.63^{1}$ & -0.80 & & & -0.68 & 2.93 & 4.31 \\
\hline 4084.498 & $26875-51351$ & 3.33 & 698 & 0.17 & -0.06 & $0.13^{2}$ & 0.08 & & & 0.09 & 3.70 & 5.08 \\
\hline 4085.011 & $22947-47420$ & 2.84 & 358 & -0.39 & -0.41 & $-0.41^{1}$ & -0.51 & & & -0.43 & 3.18 & 4.56 \\
\hline 4085.312 & $26140-50611$ & 3.24 & 559 & 0.00 & -0.34 & $-0.02^{1}$ & -0.16 & & & -0.13 & 3.48 & 4.86 \\
\hline 4085.980 & $33507-57974$ & 4.15 & 1073 & -0.83 & -0.80 & $-0.68^{1}$ & & & & -0.77 & 2.84 & 4.22 \\
\hline 4087.099 & $26875-51335$ & 3.33 & 694 & -0.47 & -0.76 & $-0.67^{2}$ & -0.70 & & & -0.65 & 2.96 & 4.34 \\
\hline 4087.801 & $29469-53925$ & 3.65 & 832 & -1.84 & & $-1.60^{1}$ & & & & -1.72 & 1.89 & 3.27 \\
\hline
\end{tabular}


TABLE 3.-Oscillator Strengths for Ultraviolet Lines of $\mathrm{Fe} \mathrm{I}$-Continued

Wave-

length Energy Low $\AA \quad K \quad$ Volts

$4088.567 \quad 29357-53808 \quad 3.64$

(2)

4090.077

4090.326

4090.984

$\begin{array}{rrr}4091.557 & 22838-47272 & 2.8 \\ 4092.287 & 29320-53749 & 3.6 \\ 4092.512 & 7377-31805 & 0.9 \\ 4095.274 & 33802-58213 & 4.1 \\ 4095.973 & 20875-45282 & 2.5\end{array}$

$4096.114 \quad 29357-53763 \quad 3.64$

$-7728-32134-0.96$

$\begin{array}{lll}4097.099 & 26479-50880 & 3.28\end{array}$

$4098.183 \quad 26140-50534 \quad 3.24$

4099.080

4100.350

4100.739

4100.916

4101.272

4101.681

4103.620

4104.132

4104.472

4104.970

4106.265

4106.437

4107.492

4109.070

4109.805

4111.060

4112.350

4112.972

4114.448
4114.957

4116.970

4117.320

4117.850

4117.870

4118.548

4118.904

4120.209

4121.805

4122.522

4123.748

4124.490
4125.622

4125.622
4125.883

4126.192

4126.880

4127.611

4127.807

4129.220

4129.474

4130.035

4132.060

4132.540
4132.902

4133.869

4134.340
$23784-48231 \quad 2.95$

$27395-51837 \quad 3.40$

$12969-37410 \quad 1.61$

$27167-51604 \quad 3.37$

$\begin{array}{ll}26140-505-51017 & 3.30\end{array}$

$34329-58710 \quad 4.26$

$6928-31307 \quad 0.86$

$19788-44166 \quad 2.45$

$27395-51771 \quad 3.40$

$20038-44411 \quad 2.48$

$26225-50587 \quad 3.25$

$26340-50699 \quad 3.26$

$24119-48476 \quad 2.99$

$26875-51229 \quad 3.33$

$20875-45221 \quad 2.59$

$27395-51740 \quad 3.40$

$26140-50475 \quad 3.24$

$26550-50880 \quad 3.29$

$22947-47272 \quad 2.84$

$26875-51192 \quad 3.33$

$27395-51705 \quad 3.40$

$33695-58002 \quad 4.18$

$22838-47136 \quad 2.83$

$27167-51462 \quad 3.37$

$26140-50423 \quad 3.24$

$24772-49053 \quad 3.07$

$27560-51837 \quad 3.42$

$34547-58825 \quad 4.28$

$28820-53094 \quad 3.57$

$26340-50611 \quad 3.26$

$24119-48383 \quad 2.99$

$22838-47093 \quad 2.83$

$22947-47197 \quad 2.84$

$21039-45282 \quad 2.61$

$29372-53610 \quad 3.64$

$34040-58272 \quad 4.22$

$22947-47177 \quad 2.84$

26875 - $51103 \quad 3.33$

$22947-47172 \quad 2.84$

$23052-47272 \quad 2.86$

$26479-50699 \quad 3.28$

$27560-51771 \quad 3.42$

$27395-51604 \quad 3.40$

$12561-36767 \quad 1.56$

$12969-37163 \quad 1.61$

$34329-58520 \quad 4.26$

$22947-47136 \quad 2.84$

$0-24181 \quad 0.00$
$22838-47177 \quad 2.83$

$27167-51351 \quad 3.37$ $\begin{array}{llllll}\text { Arc } & \text { Spk } & \mathrm{CW}(\mathrm{N}) & \mathrm{HC} & \mathrm{R}(\mathrm{N}) & \mathrm{KOC}\end{array}$

Best

Mult.

No.

$-\log g f$

$\begin{array}{rrrrrrrrr}906 & -0.74 & -0.56 & -0.81^{2} & -0.70 & -0.72 & 2.89 & 4.27 \\ 422 & -0.96 & -0.99 & -1.15^{2} & -0.94 & -1.01 & 2.60 & 3.98 \\ 700 & -0.90 & -0.89 & -1.9^{2} & -1.03 & -1.00 & 2.61 & 3.99 \\ 44 & -3.52 & & -3.55^{1} & & -3.54 & 0.07 & 1.45 \\ 695 & -0.94 & & -1.10^{2} & -1.05 & & -1.05 & 2.56 & 3.94 \\ & & & & & & & \\ 357 & -1.10 & -1.19 & -1.28^{2} & -1.28 & -1.30^{1} & -1.24 & 2.37 & 3.75 \\ & -0.68 & -0.78 & -0.75^{1} & & -0.74 & 2.87 & 4.25 \\ 18 & -3.55 & & -3.74^{2} & -3.74 & -3.69 & -0.08 & 1.30 \\ 1075 & -1.35 & & -1.19^{1} & & -1.27 & 2.34 & 3.72 \\ 217 & & -0.75 & -0.60^{2} & 0.76 & -0.68 & 2.93 & 4.31 \\ & & & & & & -0.70 & 2.91 & 4.29 \\ 911 & & & -0.70^{1} & -0.71 & -3.62 & -0.01 & 1.37 \\ 18 & & & -3.62^{1} & & -1.00 & 2.61 & 3.99 \\ 558 & & -0.92 & -1.00^{2} & -1.06 & -0.20 & 3.41 & 4.79 \\ 558 & -0.02 & -0.42 & -0.18^{2} & -0.23 & -1.92 & 1.69 & 3.07\end{array}$

$1103 \quad-1.82 \quad-1.74 \quad-1.19^{1}$

$\begin{array}{rrrrr}18 & -2.44 & -2.72 & -2.53^{2} & -2.73 \\ 173 & -1.91 & & -2.56^{1} & \\ 698 & -0.53 & -0.72 & -0.69^{2} & -0.59 \\ 120 & -1.65 & & -1.72^{2} & -1.60\end{array}$

$\begin{array}{lll}-2.59 & 1.02 & 2.40\end{array}$

$650-2.06$

$558-0.24$

$422-2.01$

$694-1.37$

$217-1.36$

697

354
559

559

558

357

689

695

1103

357

695

558

484
700

1103

801

559

423

356

356

217

$-0.61$

-0.61
0.17
-1.50

$-1.50$

$-0.62$

0.01
-1.28

$-1.77^{1}$

$-0.61^{2}$

$-1.93^{1}$

$\begin{array}{ll}-1.57^{2} & -1.27 \\ -1.51^{1} & -1.44\end{array}$

$-0.56$

-1.27
-1.44

$-0.78^{1}-0.85$

$\begin{array}{ll}0.01 & -1.60^{1}\end{array}$

$-0.14-0.16$

$-0.76^{2}-0.89$

$-0.07^{3}-0.18$

$-0.11^{1}$

$-0.6$

$-1.67$

2.97

1.94

4.35

$-1.92$

$-0.50$

1.69

3.11

$-1.97$

$-1.44$

1.64

$-1.44$

2.17

$-0.77$

2.84

0.07

$-1.46$

$-0.80$

3.68
2.15

2.15

2.81
3.50

$-1.39$

$-1.39$

$-0.87$

$-1.08^{2}$

$-1.03$

$-0.01$

-0.35
-0.77

$-0.37$

$-0.02^{2}$

$-0.47^{2}$

$-0.02$

$-0.33$

$-0.45^{1}$

$-1.63$

$-1.53^{1}$

$-1.98^{1}$

$-0.98^{1}$

$-0.78^{1}$
$0.85^{3}$

$-1.03$

0.90

1.09

$-0.96^{1}$

$-0.68$

$-0.34$

$-0.40$

$-0.43^{2}$

$-0.41$

$-0.55$

$-0.49^{2}$

$-0.65^{2}$

$-0.91-1.04$

$-1.13^{2}$

0.87

$-1.49$

1103

354

695

354

$-0.21$

$-1.04$

$-0.13$

$-1.93$

357

558

698

695

$\begin{array}{lllll}-0.13 & -0.09 & -0.11^{3} & -0.13 & -0.13^{1}\end{array}$

-0.29
-1.60

$-1.23$

$-2.68$

$-1.00$

$-0.43$

$-0.49$

$-0.60$

$-0.76$

$43-0.26$

1103

357

698
3
-0.26
-0.49

$-0.20$

$-0.36$
$-0.42$

$-0.47^{1}$

$-1.49^{2}$

$-1.21^{1}$

$-2.66^{2}$

$-1.53^{1}$

$-0.13^{1}-0.16$

$-1.17$

$-0.39$

$-1.96$

$\begin{array}{lll}-0.16^{3} & -0.09 & -0.16^{1}\end{array}$

$-0.18$

$\begin{array}{lll}-0.02^{2} & -0.06 & -0.08^{1}\end{array}$

-0.06
-0.48
-4.07
-1.01
-0.09

$-0.43$

$-0.88$

$-1.58$

$-1.98$

$-0.98$

$-0.90$

0.90

$-0.88$

$-0.41$

$-0.49$

$-0.61$

$-0.99$

$-1.51$

$-0.23$

$-1.02$

$-0.36$

$-1.84$

$-0.12$

$-0.38$

$-1.59$

$-1.22$

$-2.74$

$-0.40$

$-0$.

$-0.09$

$-0.51$

$-4.15$ 
TABLE 3.-Oscillator Strengths for Ultraviolet Lines of Fe I-Continued

\begin{tabular}{|c|c|c|c|c|c|c|c|c|c|c|c|c|c|}
\hline $\begin{array}{l}\text { Wave- } \\
\text { length } \\
\AA\end{array}$ & $\underset{K}{\text { Energy Levels }}$ & $\begin{array}{l}\text { Low } \\
\text { e.p. } \\
\text { Volts }\end{array}$ & $\begin{array}{l}\text { Mult. } \\
\text { No. }\end{array}$ & Arc & Spk & $\mathrm{CW}(\mathrm{N})$ & $\begin{array}{l}\mathrm{HC} \\
-\log \end{array}$ & $R(N)$ & $\mathrm{KOC}$ & MS & Best & $\log g f \lambda$ & $\begin{array}{l}\log \\
\mathrm{gA} / \lambda\end{array}$ \\
\hline 4134.433 & $24336-48516$ & 3.02 & 482 & & & $-0.74^{1}$ & & & & & -0.74 & 2.88 & 4.23 \\
\hline 4134.680 & $22838-47017$ & 2.83 & 357 & 0.32 & 0.05 & $0.18^{3}$ & 0.13 & $0.23^{1}$ & & & 0.18 & 3.80 & 5.15 \\
\hline 4136.512 & $27167-51335$ & 3.37 & 694 & -0.69 & & $-0.82^{2}$ & -0.96 & & & & -0.82 & 2.80 & 4.15 \\
\hline 4137.002 & $27543-51708$ & 3.41 & 726 & 0.15 & 0.22 & $0.12^{2}$ & 0.16 & & & & 0.15 & 3.77 & 5.12 \\
\hline 4137.417 & $34547-58710$ & 4.28 & 1103 & -0.89 & & $-0.82^{1}$ & & & & & -0.86 & 2.76 & 4.11 \\
\hline 4137.980 & $22846-47006$ & 2.83 & 320 & -2.38 & & & & & & & -2.38 & 1.24 & 2.59 \\
\hline 4138.840 & $18378-42533$ & 2.28 & 117 & -2.74 & & $-2.80^{1}$ & -2.79 & & & & -2.78 & 0.84 & 2.19 \\
\hline 4139.929 & $7986-32134$ & 0.99 & 18 & -2.80 & & $-2.86^{2}$ & -2.86 & & & & -2.84 & 0.78 & 2.13 \\
\hline 4140.441 & $27560-51705$ & 3.42 & 695 & -0.81 & & $-1.11^{2}$ & -1.01 & & & & -1.01 & 2.61 & 3.96 \\
\hline 4141.862 & $24339-48476$ & 3.02 & 422 & -1.03 & & $-1.04^{2}$ & -0.93 & & & & -1.01 & 2.61 & 3.96 \\
\hline 4142.625 & $34692-58825$ & 4.30 & 1103 & -0.71 & & $-0.33^{2}$ & 0.12 & & & & & & \\
\hline 4143.417 & $24575-48703$ & 3.05 & 523 & 0.58 & 0.62 & $0.61^{2}$ & 0.61 & & & & 0.61 & 4.23 & 5.58 \\
\hline 4143.870 & $12561-36686$ & 1.56 & 43 & -0.33 & & $-0.07^{3}$ & -0.02 & $-0.04^{1}$ & & -0.24 & -0.12 & 3.50 & 4.85 \\
\hline 4145.206 & $21716-45833$ & 2.69 & 274 & -1.62 & & $-1.71^{2}$ & -1.81 & & & & -1.71 & 1.91 & 3.26 \\
\hline 4146.070 & $24119-48231$ & 2.99 & 422 & -1.00 & -0.80 & $-0.87^{2}$ & -0.89 & & & & -0.89 & 2.73 & 4.08 \\
\hline 4147.347 & $26875-50980$ & 3.33 & 693 & -1.23 & & $-1.28^{1}$ & & & & & -1.26 & 2.36 & 3.71 \\
\hline 4147.672 & $11976-36079$ & 1.48 & 42 & -1.49 & -1.52 & $-1.47^{3}$ & -1.56 & & & & -1.50 & 2.12 & 3.47 \\
\hline 4149.372 & $26875-50968$ & 3.33 & 694 & & & $-0.22^{2}$ & -0.19 & & & & -0.21 & 3.41 & 4.76 \\
\hline 4149.767 & $416-24507$ & 0.05 & 3 & & & $-4.81^{1}$ & -4.84 & & & & -4.82 & -1.20 & 0.15 \\
\hline 4150.258 & $27666-51755$ & 3.43 & 695 & & & $-0.71^{2}$ & -0.62 & & & & -0.68 & 2.94 & 4.29 \\
\hline
\end{tabular}

(Paper 70A4-409) 